\title{
The divergence theorem of a triangular integral
}

Kiyohisa Tokunaga*

\section{*Correspondence:}

kiyohisa@bene.fit.ac.jp

Laboratory of Information Science, Fukuoka Institute of Technology,

Wajiro, Higashi-ku, Fukuoka,

811-0295, Japan

\begin{abstract}
This divergence theorem of a triangular integral demands the antisymmetric symbol to derive the inner product of the nabla and a vector. Replacing a cuboid (a rectangular solid) by a tetrahedron (a triangular pyramid) as the finite volume element, a single limit is only demanded for triple sums in our theory of a triple integral. The divergence theorem of a triangular integral is derived by substituting the total differentials into our new method of a triangular integral. We thus infer that our new method of a triangular integral must be the inverse operation of the total differential.
\end{abstract}

\section{Introduction}

Suppose there are three partially differentiable functions $X=X(x, y, z), Y=Y(x, y, z)$ and $Z=Z(x, y, z)$ of the three variables $x, y$ and $z$ in the 3D space. The divergence theorem in the $3 \mathrm{D}$ space is conventionally formulated as

$$
\oiint_{\partial D}(X d y d z+Y d z d x+Z d x d y)=\iiint_{D}\left(\frac{\partial X}{\partial x}+\frac{\partial Y}{\partial y}+\frac{\partial Z}{\partial z}\right) d x d y d z
$$

where $D$ is a domain of the integral and $\partial D$ is its surface area. The left-hand side of (1.1) is conventionally defined as

$$
\begin{aligned}
\oiint_{\partial D}(X d y d z+Y d z d x+Z d x d y)= & \lim _{k \rightarrow \infty} \sum_{j=1}^{k} \lim _{m \rightarrow \infty} \sum_{l=1}^{m} X\left(x_{i}, y_{j}, z_{l}\right) \Delta y_{j} \Delta z_{l} \\
& +\lim _{m \rightarrow \infty} \sum_{l=1}^{m} \lim _{n \rightarrow \infty} \sum_{i=1}^{n} Y\left(x_{i}, y_{j}, z_{l}\right) \Delta z_{l} \Delta x_{i} \\
& +\lim _{n \rightarrow \infty} \sum_{i=1}^{n} \lim _{k \rightarrow \infty} \sum_{j=1}^{k} Z\left(x_{i}, y_{j}, z_{l}\right) \Delta x_{i} \Delta y_{j},
\end{aligned}
$$

where the increments $\Delta x_{i}, \Delta y_{j}$ and $\Delta z_{l}$ are respectively introduced as $\Delta x_{i} \equiv x_{i}-x_{i-1}$, $\Delta y_{j} \equiv y_{j}-y_{j-1}$ and $\Delta z_{l} \equiv z_{l}-z_{l-1}$. The right-hand side of (1.1) is conventionally defined as

$$
\begin{aligned}
& \iiint_{D}\left(\frac{\partial X}{\partial x}+\frac{\partial Y}{\partial y}+\frac{\partial Z}{\partial z}\right) d x d y d z \\
& \quad=\lim _{n \rightarrow \infty} \sum_{i=1}^{n} \lim _{k \rightarrow \infty} \sum_{j=1}^{k} \lim _{m \rightarrow \infty} \sum_{l=1}^{m}\left(\frac{\Delta X\left[\Delta x_{i}\right]}{\Delta x_{i}}+\frac{\Delta Y\left[\Delta y_{j}\right]}{\Delta y_{j}}+\frac{\Delta Z\left[\Delta z_{l}\right]}{\Delta z_{l}}\right) \Delta x_{i} \Delta y_{j} \Delta z_{l}
\end{aligned}
$$

○ 2012 Tokunaga; licensee Springer. This is an Open Access article distributed under the terms of the Creative Commons Attribution License (http://creativecommons.org/licenses/by/2.0), which permits unrestricted use, distribution, and reproduction in any medium, provided the original work is properly cited. 
where the partial increments $\Delta X\left[\Delta x_{i}\right], \Delta Y\left[\Delta y_{j}\right]$ and $\Delta Z\left[\Delta z_{l}\right]$ are respectively introduced as $\Delta X\left[\Delta x_{i}\right] \equiv X\left(x_{i}, y_{j}, z_{l}\right)-X\left(x_{i-1}, y_{j}, z_{l}\right), \Delta Y\left[\Delta y_{j}\right] \equiv Y\left(x_{i}, y_{j}, z_{l}\right)-Y\left(x_{i}, y_{j-1}, z_{l}\right)$ and $\Delta Z\left[\Delta z_{l}\right] \equiv Z\left(x_{i}, y_{j}, z_{l}\right)-Z\left(x_{i}, y_{j}, z_{l-1}\right)$. The conventional triple integral demands triple limits at infinities $n \rightarrow \infty$ and $k \rightarrow \infty$ and $m \rightarrow \infty$ of triple independent sums $i=1,2, \ldots, n$ and $j=1,2, \ldots, k$ and $l=1,2, \ldots, m$ of rectangularly divided volumes $[1,2]$. We name this conventional triple integral as the rectangular integral for later discussion. In the divergence theorem of the conventional rectangular integral as shown in (1.1), the inner product $\nabla \cdot \mathbf{X}$ of the nabla $\nabla=\left(\frac{\partial}{\partial x}, \frac{\partial}{\partial y}, \frac{\partial}{\partial z}\right)$ and a vector $\mathbf{X}=\mathbf{X}(x, y, z)=(X, Y, Z)$, i.e.,

$$
\frac{\partial X}{\partial x}+\frac{\partial Y}{\partial y}+\frac{\partial Z}{\partial z}=\lim _{\Delta x_{i} \rightarrow 0} \frac{\Delta X\left[\Delta x_{i}\right]}{\Delta x_{i}}+\lim _{\Delta y_{j} \rightarrow 0} \frac{\Delta Y\left[\Delta y_{j}\right]}{\Delta y_{j}}+\lim _{\Delta z_{l} \rightarrow 0} \frac{\Delta Z\left[\Delta z_{l}\right]}{\Delta z_{l}}
$$

explicitly appears as an integrand shown in (1.3).

Meanwhile, the total differential is widely used even in the exterior derivative [3]. However, it is not known how to derive the divergence theorem (1.1) by substituting the total differentials into an integral formula based on the conventional rectangular triple integral method. Replacing the integrand from a scalar function to a tensor function, we may derive the divergence theorem by substituting the total differentials into an integral formula. It depends how we define a new kind of triple integral. We may introduce a new kind of the triangular triple integral by the following two properties:

1. replacing a cuboid (a rectangular solid) by a tetrahedron (a triangular pyramid);

2. replacing triple limits of independent triple sums by a single limit of dependent triple sums.

We name our new method as a triangular integral in contrast with the conventional rectangular integral. Our new method of a triangular double integral was proposed in the previous paper [4], where a rectangle is replaced by a triangle as a finite area element. Our new method of a triangular double integral is extended to triangular triple and quadruple integrals in this paper. Our triangular triple integral is possible to be formulated in the method of a single limit of triple sums. As the curl theorem was discussed by our new method of an integral in the previous paper [4], the divergence theorem is discussed by our new method of an integral in this paper.

The idea of a triangular element was already introduced in the finite element method [5]. However, the triangle is the fundamental element of an integral in our triangular integral theory.

First of all, a triangular integral on the 2D plane is briefly reviewed for simplicity. The total increments (2.33) are substituted into (2.32) to derive (2.31). Combining (2.31) with the antisymmetric finite line element vector $\left(\Delta l_{\mu}\right)_{k}$ in (2.26), we obtain (2.37). In the case of $A=B$ in (2.38), it is reduced to be the divergence theorem of a triangular integral on the 2D plane (2.36).

Next, a triangular integral is applied in the 3D space, where a cuboid (a rectangular solid) is replaced by a tetrahedron (a triangular pyramid) as the finite volume element. Our triangular triple integral only demands a single limit $n \rightarrow \infty$ of triple dependent sums $i=1,2, \ldots, j$ and $j, h=1,2, \ldots, k$ and $k=1,2, \ldots, n$ for tetrahedrally divided volumes in (3.120). We show an example in the case of a sphere. The surface area of the sphere is calculated by the limit at infinity $n \rightarrow \infty$ of the finite element method based on our triangular integral. Approximate values and their graph by this finite element method are shown in 
the case of the sphere. The divergence theorem of a triangular integral in the 3D space (3.119) is derived by calculating double dependent sums (3.95). Total increments (3.108) and (3.109) are substituted into (3.107) to derive (3.106) as the first term of (3.95). In the similar manner, (3.110) is also derived as the second term of (3.95). Combining (3.106) with the upper-right finite area element vector $\left(\Delta \sigma_{\mu}\right)_{j, k}$ in (3.86) and combining (3.110) with the lower-left finite area element vector $\left(\Delta \sigma_{\mu}\right)_{k, j}$ in (3.92), we obtain (3.120). In the case of a closed 2D surface (3.121), i.e., (3.118), it is reduced to be the main theorem (3.119) by the limit at infinity $n \rightarrow \infty$. However, the antisymmetric symbol is demanded to derive the inner product of the nabla and a vector (1.4) from the divergence theorem (3.119) based on our new method of a triangular integral, since it only implicitly appears as an integrand.

Finally, our method of a triangular integral is extended in the 4D time-space. It is apparent that the quadruple integral is necessary for the variational principle in the $4 \mathrm{D}$ timespace in physics. As the curl theorem in the $4 \mathrm{D}$ time-space based on our triangular integral was derived in the previous paper [4], the divergence theorem of a triangular integral in the $4 \mathrm{D}$ time-space (4.67) is also derived in this paper.

This paper is structured as follows. Section 2 is the preparatory part, Section 3 is the main part and Section 4 is the additional part of this paper. In Section 2, we briefly derive the divergence theorem on the 2D plane. In Section 3, we develop a theory of a scalar triple product integral by a single limit of triple sums of tetrahedrally divided volumes and derive the divergence theorem in the 3D space. In Section 4, we formulate a theory of an antisymmetric quadruple integral by a single limit of quadruple sums of hyper-triangularly divided hyper-volumes and derive the divergence theorem in the 4D time-space.

\section{Integral on the 2D plane}

The divergence theorem of a triangular integral on the 2D plane in component representation is shown in Section 2.1 and that in tensor representation is shown in Section 2.2 .

Let $f(x, y)=0$ be a piecewise smooth curve of the equation on the $x y$-plane, expressed in the Cartesian coordinates $(x, y) \in \mathbb{R}^{2}$. Assume there are two fixed points of $A\left(x_{\mathrm{A}}, y_{\mathrm{A}}\right)$ and $B\left(x_{\mathrm{B}}, y_{\mathrm{B}}\right)$. Suppose there is a sequence of points $\left\{P_{k}\left(x_{k}, y_{k}\right) \mid k=0,1,2, \ldots, n\right\}$ on $f(x, y)=$ 0 , where the initial and the terminal points are respectively $P_{0}\left(x_{0}, y_{0}\right)=A\left(x_{\mathrm{A}}, y_{\mathrm{A}}\right)$ and $P_{n}\left(x_{n}, y_{n}\right)=B\left(x_{\mathrm{B}}, y_{\mathrm{B}}\right)$.

\subsection{The divergence theorem of a triangular integral on the 2D plane in component representation}

For a triangular double integral, the increments of $x_{k}$ and $y_{k}$ for $k=1,2, \ldots, n$ are denoted as follows:

$$
\begin{aligned}
& \Delta x_{k} \equiv x_{k}-x_{k-1}, \\
& \Delta y_{k} \equiv y_{k}-y_{k-1} .
\end{aligned}
$$

Assume there are two partially differentiable functions $X=X(x, y)$ and $Y=Y(x, y)$ of the two variables $x$ and $y$ on the Cartesian coordinates of the 2D plane. Suppose there is a set of sequences $\left\{\left(X_{k}, Y_{k}\right) \mid k=0,1,2, \ldots, n\right\}$. The total increments of $X_{j}$ for $j=1,2, \ldots, k$ are 
denoted as

$$
\begin{aligned}
\Delta X_{j} & \equiv X_{j}-X_{j-1} \\
& =X\left(x_{j}, y_{j}\right)-X\left(x_{j-1}, y_{j-1}\right) .
\end{aligned}
$$

Furthermore, the increments of $x_{j}$ and $y_{j}$ for $j=1,2, \ldots, k$ are denoted as follows:

$$
\begin{gathered}
\Delta x_{j} \equiv x_{j}-x_{j-1}, \\
\Delta y_{j} \equiv y_{j}-y_{j-1} .
\end{gathered}
$$

The partial increments of $X_{j}$ for $j=1,2, \ldots, k$ are denoted as

$$
\begin{aligned}
& \Delta X\left[\Delta x_{j}\right]=X\left(x_{j}, y_{j}\right)-X\left(x_{j-1}, y_{j}\right), \\
& \Delta X\left[\Delta y_{j}\right]=X\left(x_{j-1}, y_{j}\right)-X\left(x_{j-1}, y_{j-1}\right) .
\end{aligned}
$$

Lemma 1 Let $X=X(x, y)$ be a partially differentiable function with respect to $x$ and $y$. The following holds:

$$
X_{k}=X_{0}+\sum_{j=1}^{k}\left(\frac{\Delta X\left[\Delta x_{j}\right]}{\Delta x_{j}} \Delta x_{j}+\frac{\Delta X\left[\Delta y_{j}\right]}{\Delta y_{j}} \Delta y_{j}\right)
$$

for $k=1,2, \ldots, n$.

Proof Using (2.3), $X_{k}$ for $k=1,2, \ldots, n$ is disintegrated into

$$
X_{k}=X_{0}+\sum_{j=1}^{k} \Delta X_{j}
$$

Using (2.6) and (2.7), $\Delta X_{j}$ in (2.3) is modified to be

$$
\begin{aligned}
\Delta X_{j} & =\Delta X\left[\Delta x_{j}\right]+\Delta X\left[\Delta y_{j}\right] \\
& =\frac{\Delta X\left[\Delta x_{j}\right]}{\Delta x_{j}} \Delta x_{j}+\frac{\Delta X\left[\Delta y_{j}\right]}{\Delta y_{j}} \Delta y_{j} .
\end{aligned}
$$

Substituting (2.10) into (2.9), we obtain (2.8).

Q.E.D.

Lemma 2 Let $Y=Y(x, y)$ be a partially differentiable function with respect to $x$ and $y$. The following holds:

$$
Y_{k}=Y_{0}+\sum_{j=1}^{k}\left(\frac{\Delta Y\left[\Delta x_{j}\right]}{\Delta x_{j}} \Delta x_{j}+\frac{\Delta Y\left[\Delta y_{j}\right]}{\Delta y_{j}} \Delta y_{j}\right)
$$

for $k=1,2, \ldots, n$.

Proof In the similar manner, we obtain (2.11).

Q.E.D. 
Definition 1 A single integral on the 2D plane in component representation $\int_{f(x, y)=0}^{[A, B]}(X d y-$ $Y d x)$ is defined in the case of a piecewise smooth curve of the equation $f(x, y)=0$ by the formula

$$
\int_{f(x, y)=0}^{[A, B]}(X d y-Y d x)=\lim _{n \rightarrow \infty} \sum_{k=1}^{n}\left(X_{k} \Delta y_{k}-Y_{k} \Delta x_{k}\right)
$$

where $\left(x_{A}, y_{A}\right)=\left(x_{0}, y_{0}\right)$ and $\left(x_{B}, y_{B}\right)=\left(x_{n}, y_{n}\right)$.

Our triangular double integral and the divergence theorem of a triangular integral on the $2 \mathrm{D}$ plane in component representation are shown as follows.

Definition 2 A triangular double integral, integrands of which are partial differentials on the $2 \mathrm{D}$ plane in component representation $\iint_{f(x, y) \leq 0}^{[A, B]}\left[\left(\frac{\partial X^{\prime}}{\partial x^{\prime}} d x^{\prime}+\frac{\partial X^{\prime}}{\partial y^{\prime}} d y^{\prime}\right) d y-\left(\frac{\partial Y^{\prime}}{\partial x^{\prime}} d x^{\prime}+\right.\right.$ $\left.\left.\frac{\partial Y^{\prime}}{\partial y^{\prime}} d y^{\prime}\right) d x\right]$, is defined in the case of a piecewise smooth curve of the equation $f(x, y)=0$ by the formula

$$
\begin{gathered}
\iint_{f(x, y) \leq 0}^{[A, B]}\left[\left(\frac{\partial X^{\prime}}{\partial x^{\prime}} d x^{\prime}+\frac{\partial X^{\prime}}{\partial y^{\prime}} d y^{\prime}\right) d y-\left(\frac{\partial Y^{\prime}}{\partial x^{\prime}} d x^{\prime}+\frac{\partial Y^{\prime}}{\partial y^{\prime}} d y^{\prime}\right) d x\right] \\
=\lim _{n \rightarrow \infty} \sum_{k=1}^{n} \sum_{j=1}^{k}\left[\left(\frac{\Delta X\left[\Delta x_{j}\right]}{\Delta x_{j}} \Delta x_{j}+\frac{\Delta X\left[\Delta y_{j}\right]}{\Delta y_{j}} \Delta y_{j}\right) \Delta y_{k}\right. \\
\left.-\left(\frac{\Delta Y\left[\Delta x_{j}\right]}{\Delta x_{j}} \Delta x_{j}+\frac{\Delta Y\left[\Delta y_{j}\right]}{\Delta y_{j}} \Delta y_{j}\right) \Delta x_{k}\right],
\end{gathered}
$$

where $\left(x_{A}, y_{A}\right)=\left(x_{0}, y_{0}\right)$ and $\left(x_{B}, y_{B}\right)=\left(x_{n}, y_{n}\right)$.

Theorem 1 Assume $\partial D$ is a piecewise smooth curve of the equation $f(x, y)=0$ on the $x y$ plane, expressed in the Cartesian coordinates $(x, y) \in \mathbb{R}^{2}$. Assume $D$ is the region inside and on $\partial D$. Let $X=X(x, y)$ and $Y=Y(x, y)$ be partially differentiable functions with respect to $x$ and $y$ in $D$. In the case of a closed integral path, i.e., $A\left(x_{A}, y_{A}\right)=B\left(x_{B}, y_{B}\right)$, the divergence theorem of a triangular integral on the $2 D$ plane holds:

$$
\begin{aligned}
& \oint_{\partial D}(X d y-Y d x) \\
& \quad=\iint_{D}\left(\frac{\partial X^{\prime}}{\partial x^{\prime}} d x^{\prime} d y+\frac{\partial X^{\prime}}{\partial y^{\prime}} d y^{\prime} d y-\frac{\partial Y^{\prime}}{\partial x^{\prime}} d x^{\prime} d x-\frac{\partial Y^{\prime}}{\partial y^{\prime}} d y^{\prime} d x\right) .
\end{aligned}
$$

Proof Combining (2.8) with $\Delta y_{k}$ and (2.11) with $\Delta x_{k}$ for the sum of $k=1,2, \ldots, n$, the following holds:

$$
\begin{aligned}
\sum_{k=1}^{n}\left(X_{k} \Delta y_{k}-Y_{k} \Delta x_{k}\right)= & \sum_{k=1}^{n} \sum_{j=1}^{k}\left[\left(\frac{\Delta X\left[\Delta x_{j}\right]}{\Delta x_{j}} \Delta x_{j}+\frac{\Delta X\left[\Delta y_{j}\right]}{\Delta y_{j}} \Delta y_{j}\right) \Delta y_{k}\right. \\
& \left.-\left(\frac{\Delta Y\left[\Delta x_{j}\right]}{\Delta x_{j}} \Delta x_{j}+\frac{\Delta Y\left[\Delta y_{j}\right]}{\Delta y_{j}} \Delta y_{j}\right) \Delta x_{k}\right] \\
& +X_{0}\left(y_{n}-y_{0}\right)-Y_{0}\left(x_{n}-x_{0}\right) .
\end{aligned}
$$


The limit of (2.15) at infinity $n \rightarrow \infty$ is expressed as

$$
\begin{aligned}
\int_{f(x, y)=0}^{[A, B]}(X d y-Y d x)= & \iint_{f(x, y) \leq 0}^{[A, B]}\left[\left(\frac{\partial X^{\prime}}{\partial x^{\prime}} d x^{\prime}+\frac{\partial X^{\prime}}{\partial y^{\prime}} d y^{\prime}\right) d y-\left(\frac{\partial Y^{\prime}}{\partial x^{\prime}} d x^{\prime}+\frac{\partial Y^{\prime}}{\partial y^{\prime}} d y^{\prime}\right) d x\right] \\
& +X_{A}\left(y_{B}-y_{A}\right)-Y_{A}\left(x_{B}-x_{A}\right)
\end{aligned}
$$

by Definitions 1 and 2, where $X_{A}=X\left(x_{A}, y_{A}\right)$ and $Y_{A}=Y\left(x_{A}, y_{A}\right)$. In the case of

$$
X_{A}\left(y_{B}-y_{A}\right)-Y_{A}\left(x_{B}-x_{A}\right)=0,
$$

(2.16) is reduced to be (2.14). The coincidence of the initial and the terminal points, i.e., $A=B$ satisfies this condition (2.17).

Q.E.D.

Since the inner product of the nabla and a vector does not explicitly appear in Theorem 1 , the corollary shown below is necessary to be derived. Infinitesimal area element $d^{2} \sigma$ is introduced as $d^{2} \sigma=-\frac{1}{2}\left(d x d y^{\prime}-d y d x^{\prime}\right)$.

Corollary 1 In the case of

$$
\oint_{\partial D}(X d y-Y d x)=\iint_{D} \rho d^{2} \sigma
$$

where $\rho=\rho(x, y)$ is a given function, the following holds:

$$
\frac{\partial X}{\partial x}+\frac{\partial Y}{\partial y}=\rho .
$$

Proof Using Theorem 1, the left-hand side of (2.18) is modified to be

$$
\begin{aligned}
& \iint_{D}\left(\frac{\partial X^{\prime}}{\partial x^{\prime}} d x^{\prime} d y+\frac{\partial X^{\prime}}{\partial y^{\prime}} d y^{\prime} d y-\frac{\partial Y^{\prime}}{\partial x^{\prime}} d x^{\prime} d x-\frac{\partial Y^{\prime}}{\partial y^{\prime}} d y^{\prime} d x\right) \\
& \quad=\iint_{D} \frac{\rho}{2}\left(d y d x^{\prime}-d x d y^{\prime}\right) .
\end{aligned}
$$

Therefore, the following hold:

$$
\begin{aligned}
& \iint_{D} \frac{\partial X^{\prime}}{\partial x^{\prime}} d x^{\prime} d y=\iint_{D} \frac{\rho}{2} d x^{\prime} d y \\
& \iint_{D} \frac{\partial Y^{\prime}}{\partial y^{\prime}} d y^{\prime} d x=\iint_{D} \frac{\rho}{2} d y^{\prime} d x
\end{aligned}
$$

Combining (2.21) with (2.22), they are reduced to be (2.19).

Q.E.D.

Remark In an explicit differential form, the following holds:

$$
\frac{\partial X^{\prime}}{\partial x^{\prime}}+\frac{\partial Y^{\prime}}{\partial y^{\prime}}=\frac{\partial X}{\partial x}+\frac{\partial Y}{\partial y} .
$$

However, (2.23) does not hold as an integrand in our triangular integral. 


\subsection{The divergence theorem of a triangular integral on the 2D plane in tensor representation}

Before expressing the following formulae in tensor representation, the antisymmetric symbol on the 2D plane is introduced as

$$
\varepsilon^{\alpha \beta}=\varepsilon_{\alpha \beta}= \begin{cases}+1 & \text { for } \varepsilon^{12}=\varepsilon_{12} \\ -1 & \text { for } \varepsilon^{21}=\varepsilon_{21} \\ 0 & \text { otherwise. }\end{cases}
$$

On the 2D plane, $\varepsilon_{\alpha \mu} \varepsilon^{\alpha \beta}=\delta_{\mu}^{\beta}$ holds for $\beta, \mu=1,2$, where the index is summed over $\alpha=1,2$. $\frac{1}{2} \varepsilon_{\alpha \beta} \varepsilon^{\alpha \beta}=1$ also holds, where the indices are summed over $\alpha, \beta=1,2$.

Denoting $x^{1}=x$ and $x^{2}=y$, the increments of $\left(x^{\alpha}\right)_{k}$ for $\alpha=1,2$ and $k=1,2, \ldots, n$ are expressed as

$$
\Delta\left(x^{\alpha}\right)_{k} \equiv\left(x^{\alpha}\right)_{k}-\left(x^{\alpha}\right)_{k-1} .
$$

Using the antisymmetric symbol shown in (2.24), the antisymmetric finite line element vector $\left(\Delta l_{\mu}\right)_{k}$ is introduced as

$$
\begin{aligned}
\left(\Delta l_{\mu}\right)_{k} & =\varepsilon_{\alpha \mu}\left(\overrightarrow{P_{k} P_{k-1}}\right)^{\alpha} \\
& =-\varepsilon_{\alpha \mu} \Delta\left(x^{\alpha}\right)_{k}
\end{aligned}
$$

for $\mu=1,2$ and $k=1,2, \ldots, n$, where $\left(\overrightarrow{P_{k} P_{k-1}}\right)^{\alpha}$ is $\alpha$-component of $\overrightarrow{P_{k} P_{k-1}}$, and the index is summed over $\alpha=1,2$. We denote $X^{1}=X$ and $X^{2}=Y$ in the following. Assume there are two partially differentiable functions $X^{\mu}=X^{\mu}\left(x^{\beta}\right)$ of two variables $x^{\beta}$ for $\beta, \mu=1,2$ on the Cartesian coordinates of the 2D plane. The total increments of $\left(X^{\mu}\right)_{j}$ for $\mu=1,2$ and $j=1,2, \ldots, k$ are denoted as

$$
\begin{aligned}
\Delta\left(X^{\mu}\right)_{j} & \equiv\left(X^{\mu}\right)_{j}-\left(X^{\mu}\right)_{j-1} \\
& =X^{\mu}\left(x_{j}, y_{j}\right)-X^{\mu}\left(x_{j-1}, y_{j-1}\right) .
\end{aligned}
$$

The increments of $\left(x^{\beta}\right)_{j}$ for $\beta=1,2$ and $j=1,2, \ldots, k$ are expressed as

$$
\Delta\left(x^{\beta}\right)_{j} \equiv\left(x^{\beta}\right)_{j}-\left(x^{\beta}\right)_{j-1} .
$$

The partial increments of $\left(X^{\mu}\right)_{j}$ for $\mu=1,2$ and $j=1,2, \ldots, k$ are denoted as

$$
\begin{aligned}
& \Delta X^{\mu}\left[\Delta x_{j}\right] \equiv X^{\mu}\left(x_{j}, y_{j}\right)-X^{\mu}\left(x_{j-1}, y_{j}\right), \\
& \Delta X^{\mu}\left[\Delta y_{j}\right] \equiv X^{\mu}\left(x_{j-1}, y_{j}\right)-X^{\mu}\left(x_{j-1}, y_{j-1}\right) .
\end{aligned}
$$

Lemma 3 Let $X^{\mu}=X^{\mu}\left(x^{\beta}\right)$ be partially differentiable functions with respect to $x^{\beta}$ for $\beta, \mu=$ 1,2. The following holds:

$$
\left(X^{\mu}\right)_{k}=\left(X^{\mu}\right)_{0}+\sum_{j=1}^{k} \frac{\Delta X^{\mu}\left[\Delta\left(x^{\beta}\right)_{j}\right]}{\Delta\left(x^{\beta}\right)_{j}} \Delta\left(x^{\beta}\right)_{j}
$$

for $\mu=1,2$ and $k=1,2, \ldots, n$, where the index is summed over $\beta=1,2$. 
Proof Using (2.27), $\left(X^{\mu}\right)_{k}$ for $\mu=1,2$ and $k=1,2, \ldots, n$ is disintegrated into

$$
\left(X^{\mu}\right)_{k}=\left(X^{\mu}\right)_{0}+\sum_{j=1}^{k} \Delta\left(X^{\mu}\right)_{j}
$$

Using (2.29) and (2.30), $\Delta\left(X^{\mu}\right)_{j}$ for $\mu=1,2$ and $j=1,2, \ldots, k$ in (2.27) is modified to be

$$
\begin{aligned}
\Delta\left(X^{\mu}\right)_{j} & =\Delta X^{\mu}\left[\Delta x_{j}\right]+\Delta X^{\mu}\left[\Delta y_{j}\right] \\
& =\frac{\Delta X^{\mu}\left[\Delta\left(x^{\beta}\right)_{j}\right]}{\Delta\left(x^{\beta}\right)_{j}} \Delta\left(x^{\beta}\right)_{j},
\end{aligned}
$$

where the index is summed over $\beta=1,2$. Substituting (2.33) into (2.32), we obtain (2.31).

Q.E.D.

Definition 3 A single integral on the 2D plane in tensor representation $\int_{f(x, y)=0}^{[A, B]} X^{\mu} d l_{\mu}$ is defined in the case of a piecewise smooth curve of the equation $f(x, y)=0$ by the formula

$$
\int_{f(x, y)=0}^{[A, B]} X^{\mu} d l_{\mu}=\lim _{n \rightarrow \infty} \sum_{k=1}^{n}\left(X^{\mu}\right)_{k}\left(\Delta l_{\mu}\right)_{k}
$$

where $\left(x_{A}, y_{A}\right)=\left(x_{0}, y_{0}\right)$ and $\left(x_{B}, y_{B}\right)=\left(x_{n}, y_{n}\right)$ and the index is summed over $\mu=1,2$.

Our triangular double integral and the divergence theorem of a triangular integral on the 2D plane in tensor representation are shown as follows.

Definition 4 A triangular double integral, integrands of which are partial differentials on the 2D plane in tensor representation $\iint_{f(x, y) \leq 0}^{[A, B]} \frac{\partial X^{\prime \mu}}{\partial x^{\prime \beta}} d x^{\prime \beta} d l_{\mu}$, is defined in the case of a piecewise smooth curve of the equation $f(x, y)=0$ by the formula,

$$
\iint_{f(x, y) \leq 0}^{[A, B]} \frac{\partial X^{\prime \mu}}{\partial x^{\prime \beta}} d x^{\prime \beta} d l_{\mu}=\lim _{n \rightarrow \infty} \sum_{k=1}^{n} \sum_{j=1}^{k} \frac{\Delta X^{\mu}\left[\Delta\left(x^{\beta}\right)_{j}\right]}{\Delta\left(x^{\beta}\right)_{j}} \Delta\left(x^{\beta}\right)_{j}\left(\Delta l_{\mu}\right)_{k},
$$

where $\left(x_{A}, y_{A}\right)=\left(x_{0}, y_{0}\right)$ and $\left(x_{B}, y_{B}\right)=\left(x_{n}, y_{n}\right)$ and the indices are summed over $\beta, \mu=1,2$.

Theorem 2 Assume $\partial D$ is a piecewise smooth curve of the equation $f(x, y)=0$ on the $x y$ plane, expressed in the Cartesian coordinates $(x, y) \in \mathbb{R}^{2}$. Assume $D$ is the region inside and on $\partial D$. Let $X^{1}=X=X(x, y)$ and $X^{2}=Y=Y(x, y)$ be partially differentiable functions with respect to $x^{1}=x$ and $x^{2}=y$ in $D$. In the case of a closed integral path, i.e., $A\left(x_{A}, y_{A}\right)=$ $B\left(x_{B}, y_{B}\right)$, the divergence theorem of a triangular integral on the $2 D$ plane holds:

$$
\oint_{\partial D} X^{\mu} d l_{\mu}=\iint_{D} \frac{\partial X^{\prime \mu}}{\partial x^{\prime \beta}} d x^{\prime \beta} d l_{\mu}
$$

where $d l_{\mu}=-\varepsilon_{\alpha \mu} d x^{\alpha}$ and the indices are summed over $\alpha, \beta, \mu=1,2$. 
Proof Combining (2.31) with the antisymmetric finite line element vector $\left(\Delta l_{\mu}\right)_{k}=$ $-\varepsilon_{\alpha \mu} \Delta\left(x^{\alpha}\right)_{k}$ in (2.26) for the sum of $k=1,2, \ldots, n$, the following holds:

$$
\begin{aligned}
& \sum_{k=1}^{n}\left(X^{\mu}\right)_{k}\left(\Delta l_{\mu}\right)_{k} \\
& \quad=\sum_{k=1}^{n} \sum_{j=1}^{k} \frac{\Delta\left(X^{\mu}\right)\left[\Delta\left(x^{\beta}\right)_{j}\right]}{\Delta\left(x^{\beta}\right)_{j}} \Delta\left(x^{\beta}\right)_{j}\left(\Delta l_{\mu}\right)_{k}-\varepsilon_{\alpha \mu}\left(X^{\mu}\right)_{0}\left[\left(x^{\alpha}\right)_{n}-\left(x^{\alpha}\right)_{0}\right],
\end{aligned}
$$

where the indices are summed over $\alpha, \beta, \mu=1,2$. The limit of (2.37) at infinity $n \rightarrow \infty$ is expressed as

$$
\int_{f(x, y)=0}^{[A, B]} X^{\mu} d l_{\mu}=\iint_{f(x, y) \leq 0}^{[A, B]} \frac{\partial X^{\prime \mu}}{\partial x^{\prime \beta}} d x^{\prime \beta} d l_{\mu}-\varepsilon_{\alpha \mu} X^{\mu}(A)\left[x^{\alpha}(B)-x^{\alpha}(A)\right],
$$

by Definitions 3 and 4 , where $\left(x^{\alpha}\right)_{0}=x^{\alpha}(A),\left(x^{\alpha}\right)_{n}=x^{\alpha}(B),\left(X^{\mu}\right)_{0}=X^{\mu}(A)$ and the indices are summed over $\alpha, \beta, \mu=1,2$. In the case of

$$
\varepsilon_{\alpha \mu} X^{\mu}(A)\left[x^{\alpha}(B)-x^{\alpha}(A)\right]=0,
$$

where the indices are summed over $\alpha, \mu=1,2,(2.38)$ is reduced to be (2.36). The coincidence of the initial and the terminal points, i.e., $A=B$ satisfies the condition (2.39). Q.E.D.

Infinitesimal area element $d^{2} \sigma$ is introduced as $d^{2} \sigma=\frac{1}{2} d x^{\prime \beta} d l_{\beta}$, where the index is summed over $\beta=1,2$.

\section{Corollary 2 In the case of}

$$
\oint_{\partial D} X^{\mu} d l_{\mu}=\iint_{D} \rho d^{2} \sigma
$$

where $\rho=\rho(x, y)$ is a given function and the index is summed over $\mu=1,2$, the following holds:

$$
\frac{\partial X^{\mu}}{\partial x^{\mu}}=\rho .
$$

Proof Using Theorem 2, the left-hand side of (2.40) is modified to be

$$
\iint_{D} \frac{\partial X^{\prime \mu}}{\partial x^{\prime \beta}} \varepsilon_{\alpha \mu} d x^{\alpha} d x^{\prime \beta}=\iint_{D} \frac{\rho}{2} \varepsilon_{\alpha \beta} d x^{\alpha} d x^{\prime \beta},
$$

where the indices are summed over $\alpha, \beta, \mu=1,2$. In order to hold (2.42) for any value of integral variables, the following formula is demanded:

$$
\frac{\partial X^{\prime \mu}}{\partial X^{\prime \beta}} \varepsilon_{\alpha \mu}=\frac{\rho}{2} \varepsilon_{\alpha \beta}
$$

for $\alpha, \beta=1,2$, where the index is summed over $\mu=1,2$. Multiplying $\varepsilon^{\alpha \beta}$ to the both sides of the formula (2.43), it is reduced to be the differential equation (2.41).

Q.E.D.

Remark $X^{\prime \mu}$ and $x^{\prime \mu}$ are respectively replaced by $X^{\mu}$ and $x^{\mu}$ in the case they are explicit differential forms, but not as integrand. 


\section{Integral in the 3D space}

As the curl theorem in the 3D space was derived in the previous paper [4], the divergence theorem of a triangular integral in the 3D space is derived in this paper.

Let $g(x, y, z)=0$ be a piecewise smooth surface of the equation in the $x y z$-space, expressed in the Cartesian coordinates $(x, y, z) \in \mathbb{R}^{3}$. Suppose there are two kinds of double sequences of points $\left\{P_{j, k}\right\}$ and $\left\{P_{k, j}\right\}$ for $j=0,1,2, \ldots, k$ and $k=0,1,2, \ldots, n$ on $g(x, y, z)=0$. The surface area of the sphere is approximated as a finite number of pieces of the plane. Dividing these pieces of plane by upper-right part and lower-left one, we may express them as the two kinds of double sums of areas. Each piece of the plane is an isosceles trapezium. It is divided into two triangles, upper-right and lower-left ones.

\subsection{Two kinds of finite area element vectors in the 3D space in component representation}

1. The upper-right finite area element vector $\overrightarrow{\Delta^{2} \sigma_{j, k}}$.

Let the coordinates of $O, P_{j, k}, P_{j-1, k}, P_{j-1, k-1}$ and $P_{j, k-1}$ respectively be $(0,0,0),\left(x_{j, k}, y_{j, k}\right.$, $\left.z_{j, k}\right),\left(x_{j-1, k}, y_{j-1, k}, z_{j-1, k}\right),\left(x_{j-1, k-1}, y_{j-1, k-1}, z_{j-1, k-1}\right)$ and $\left(x_{j, k-1}, y_{j, k-1}, z_{j, k-1}\right)$ for $j=1,2, \ldots, k$ and $k=1,2, \ldots, n$. An upper-right finite area element vector $\overrightarrow{\Delta^{2} \sigma_{j, k}}$ for $j=1,2, \ldots, k$ and $k=$ $1,2, \ldots, n$ is disintegrated into two parts,

$$
\overrightarrow{\Delta^{2} \sigma_{j, k}}=\overrightarrow{\Delta_{j}^{2} \sigma_{j, k}}+\overrightarrow{\Delta_{k}^{2} \sigma_{j, k}}
$$

where $\overrightarrow{\Delta_{j}^{2} \sigma_{j, k}}$ and $\overrightarrow{\Delta_{k}^{2} \sigma_{j, k}}$ are respectively introduced as

$$
\begin{aligned}
& \overrightarrow{\Delta_{j}^{2} \sigma_{j, k}}=\frac{1}{2} \overrightarrow{P_{j, k} P_{j-1, k}} \times \overrightarrow{P_{j, k} P_{j-1, k-1}}, \\
& \overrightarrow{\Delta_{k}^{2} \sigma_{j, k}}=\frac{1}{2} \overrightarrow{P_{j, k} P_{j-1, k-1}} \times \overrightarrow{P_{j, k} P_{j, k-1}}
\end{aligned}
$$

as shown in Figures 1 and 2.

2. The lower-left finite area element vector $\overrightarrow{\Delta^{2} \sigma_{k, j}}$.

Let the coordinates of $O, P_{k, j}, P_{k-1, j}, P_{k-1, j-1}$ and $P_{k, j-1}$ respectively be $(0,0,0),\left(x_{k, j}, y_{k, j}\right.$, $\left.z_{k, j}\right),\left(x_{k-1, j}, y_{k-1, j}, z_{k-1, j}\right),\left(x_{k-1, j-1}, y_{k-1, j-1}, z_{k-1, j-1}\right)$ and $\left(x_{k, j-1}, y_{k, j-1}, z_{k, j-1}\right)$ for $j=1,2, \ldots, k$ and $k=1,2, \ldots, n$. A lower-left finite area element vector $\overrightarrow{\Delta^{2} \sigma_{k, j}}$ for $j=1,2, \ldots, k$ and $k=$ $1,2, \ldots, n$ is disintegrated into two parts,

$$
\overrightarrow{\Delta^{2} \sigma_{k, j}}=\overrightarrow{\Delta_{k}^{2} \sigma_{k, j}}+\overrightarrow{\Delta_{j}^{2} \sigma_{k, j}}
$$

where $\overrightarrow{\Delta_{k}^{2} \sigma_{k, j}}$ and $\overrightarrow{\Delta_{j}^{2} \sigma_{k, j}}$ are respectively introduced as

$$
\begin{aligned}
& \overrightarrow{\Delta_{k}^{2} \sigma_{k, j}}=\frac{1}{2} \overrightarrow{P_{k, j} P_{k-1, j}} \times \overrightarrow{P_{k, j} P_{k-1, j-1}}, \\
& \overrightarrow{\Delta_{j}^{2} \sigma_{k, j}}=\frac{1}{2} \overrightarrow{P_{k, j} P_{k-1, j-1}} \times \overrightarrow{P_{k, j} P_{k, j-1}}
\end{aligned}
$$

as shown in Figures 3 and 4.

Component representations of $\overrightarrow{\Delta_{j}^{2} \sigma_{j, k}}, \overrightarrow{\Delta_{k}^{2} \sigma_{j, k}}, \overrightarrow{\Delta_{k}^{2} \sigma_{k, j}}$ and $\overrightarrow{\Delta_{j}^{2} \sigma_{k, j}}$ are shown in the following. 


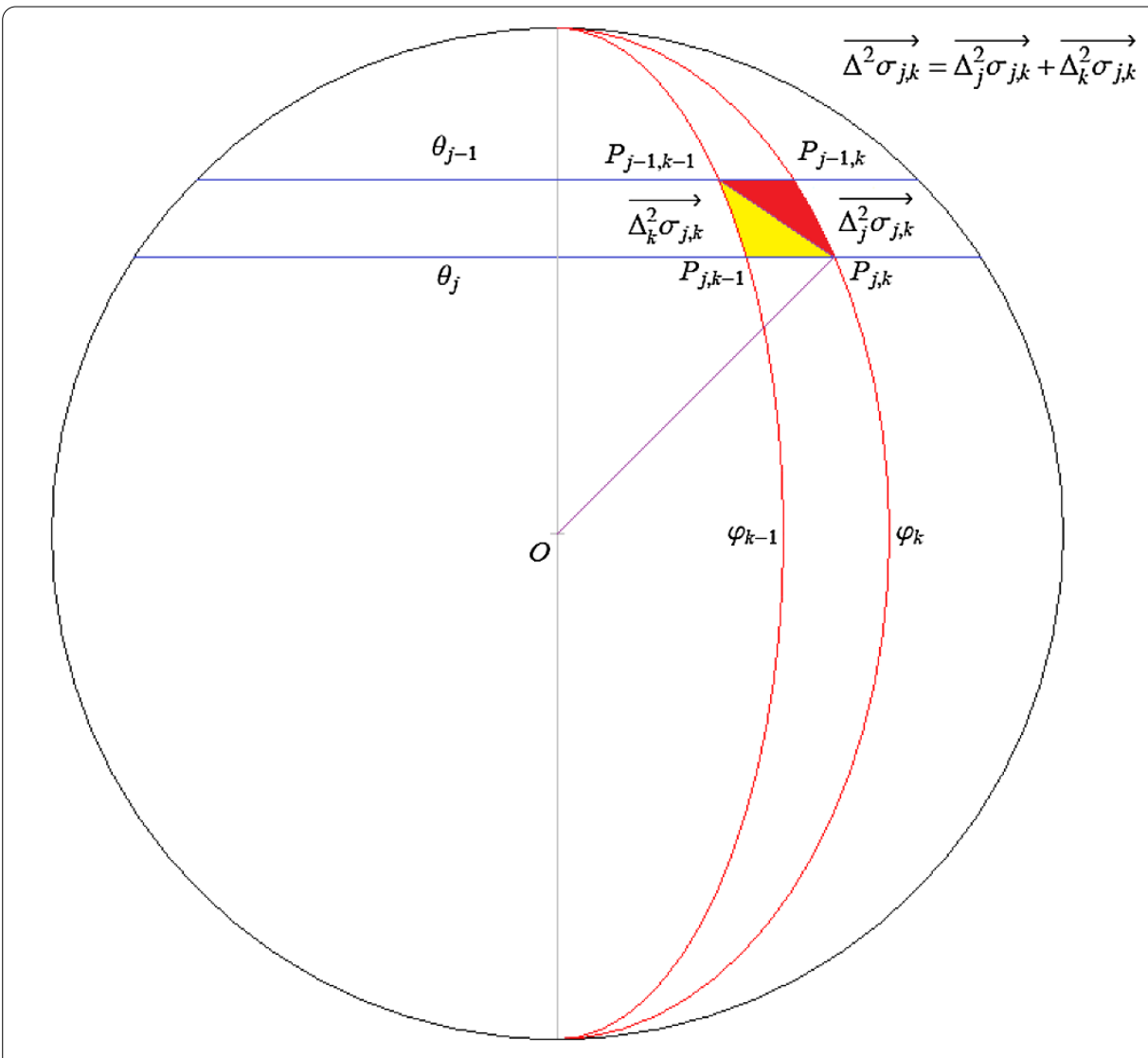

Figure 1 An upper-right piece of a divided area $\overrightarrow{\Delta^{2} \sigma_{j, k}}$. An upper-right finite area element vector $\overrightarrow{\Delta^{2} \sigma_{j, k}}$ is disintegrated into two parts,

$$
\overrightarrow{\Delta^{2} \sigma_{j, k}}=\overrightarrow{\Delta_{j}^{2} \sigma_{j, k}}+\overrightarrow{\Delta_{k}^{2} \sigma_{j, k}}
$$

for $j=1,2, \ldots, k$ and $k=1,2, \ldots, n$.

1. The upper-right finite area element vector $\overrightarrow{\Delta^{2} \sigma_{j, k}}$.

(a) Component representation of $\overrightarrow{\Delta_{j}^{2} \sigma_{j, k}}$.

$\overrightarrow{\Delta_{j}^{2} \sigma_{j, k}}$ in (3.2) for $j=1,2, \ldots, k$ and $k=1,2, \ldots, n$ is expressed as

$$
\begin{aligned}
\overrightarrow{\Delta_{j}^{2} \sigma_{j, k}} & =\frac{1}{2}\left(\begin{array}{l}
x_{j-1, k}-x_{j, k} \\
y_{j-1, k}-y_{j, k} \\
z_{j-1, k}-z_{j, k}
\end{array}\right) \times\left(\begin{array}{l}
x_{j-1, k-1}-x_{j, k} \\
y_{j-1, k-1}-y_{j, k} \\
z_{j-1, k-1}-z_{j, k}
\end{array}\right) \\
& =\frac{1}{2}\left(\begin{array}{l}
y_{j, k}\left(z_{j-1, k}-z_{j-1, k-1}\right)+y_{j-1, k-1}\left(z_{j, k}-z_{j-1, k}\right)-y_{j-1, k}\left(z_{j, k}-z_{j-1, k-1}\right) \\
z_{j, k}\left(x_{j-1, k}-x_{j-1, k-1}\right)+z_{j-1, k-1}\left(x_{j, k}-x_{j-1, k}\right)-z_{j-1, k}\left(x_{j, k}-x_{j-1, k-1}\right) \\
x_{j, k}\left(y_{j-1, k}-y_{j-1, k-1}\right)+x_{j-1, k-1}\left(y_{j, k}-y_{j-1, k}\right)-x_{j-1, k}\left(y_{j, k}-y_{j-1, k-1}\right)
\end{array}\right) .
\end{aligned}
$$

Denoting $x^{1}=x, x^{2}=y$ and $x^{3}=z$, we introduce $\Delta_{j}\left(x^{\mu}\right)_{j, k}$ and $\Delta_{k}\left(x^{\mu}\right)_{j-1, k}$ for $\mu=1,2,3$ and $j=1,2, \ldots, k$ and $k=1,2, \ldots, n$ as

$$
\Delta_{j}\left(x^{\mu}\right)_{j, k} \equiv\left(x^{\mu}\right)_{j, k}-\left(x^{\mu}\right)_{j-1, k},
$$




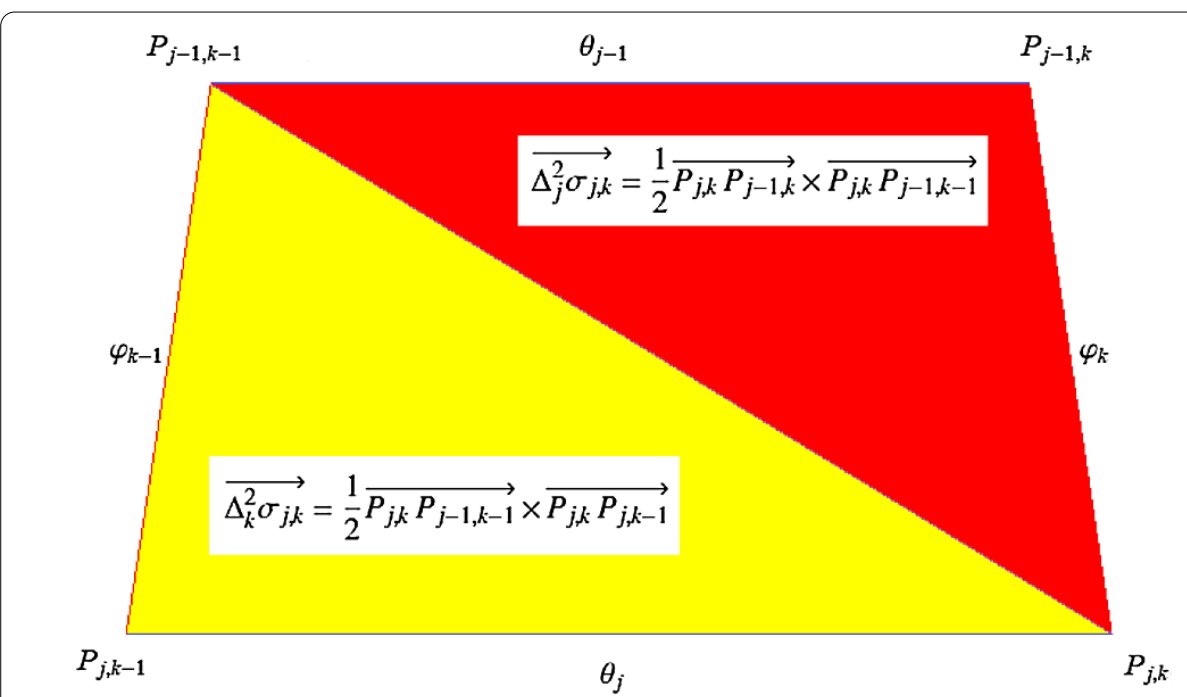

Figure 2 Two kinds of the finite area element of an upper-right piece. Two kinds of finite area element vectors $\overrightarrow{\Delta_{j}^{2} \sigma_{j, k}}$ and $\overrightarrow{\Delta_{k}^{2} \sigma_{j, k}}$ in (3.1) shown in Figure 1 are introduced as

$$
\begin{aligned}
& \overrightarrow{\Delta_{j}^{2} \sigma_{j, k}}=\frac{1}{2} \overrightarrow{P_{j, k} P_{j-1, k}} \times \overrightarrow{P_{j, k} P_{j-1, k-1},} \\
& \overrightarrow{\Delta_{k}^{2} \sigma_{j, k}}=\frac{1}{2} \overrightarrow{P_{j, k} P_{j-1, k-1}} \times \overrightarrow{P_{j, k} P_{j, k-1}} \\
& \text { for } j=1,2, \ldots, k \text { and } k=1,2, \ldots, n .
\end{aligned}
$$

$$
\Delta_{k}\left(x^{\mu}\right)_{j-1, k} \equiv\left(x^{\mu}\right)_{j-1, k}-\left(x^{\mu}\right)_{j-1, k-1} .
$$

The concrete forms of $\Delta_{j} x_{j, k}, \Delta_{j} y_{j, k}, \Delta_{j} z_{j, k}, \Delta_{k} x_{j-1, k}, \Delta_{k} y_{j-1, k}$ and $\Delta_{k} z_{j-1, k}$ are respectively shown in (3.44), (3.45), (3.46), (3.47), (3.48) and (3.49) in the case of the sphere. Substituting (3.8) and (3.9) into (3.7), we modify the $x$-component of $\overrightarrow{\Delta_{j}^{2} \sigma_{j, k}}$ for $j=1,2, \ldots, k$ and $k=1,2, \ldots, n$ as

$$
\begin{aligned}
\left(\overrightarrow{\Delta_{j}^{2} \sigma_{j, k}}\right)_{x} & =\frac{1}{2}\left[y_{j, k} \Delta_{k} z_{j-1, k}+y_{j-1, k-1} \Delta_{j} z_{j, k}-y_{j-1, k}\left(\Delta_{j} z_{j, k}+\Delta_{k} z_{j-1, k}\right)\right] \\
& =\frac{1}{2}\left[\left(y_{j, k}-y_{j-1, k}\right) \Delta_{k} z_{j-1, k}-\left(y_{j-1, k}-y_{j-1, k-1}\right) \Delta_{j} z_{j, k}\right] \\
& =\frac{1}{2}\left(\Delta_{j} y_{j, k} \Delta_{k} z_{j-1, k}-\Delta_{j} z_{j, k} \Delta_{k} y_{j-1, k}\right) .
\end{aligned}
$$

In the similar manner, we respectively modify the $y$-and the $z$-components of (3.7) for $j=1,2, \ldots, k$ and $k=1,2, \ldots, n$ as

$$
\begin{aligned}
\left(\overrightarrow{\Delta_{j}^{2} \sigma_{j, k}}\right)_{y} & =\frac{1}{2}\left(\Delta_{j} z_{j, k} \Delta_{k} x_{j-1, k}-\Delta_{j} x_{j, k} \Delta_{k} z_{j-1, k}\right), \\
\left(\overrightarrow{\Delta_{j}^{2} \sigma_{j, k}}\right)_{z} & =\frac{1}{2}\left(\Delta_{j} x_{j, k} \Delta_{k} y_{j-1, k}-\Delta_{j} y_{j, k} \Delta_{k} x_{j-1, k}\right) .
\end{aligned}
$$

(b) Component representation of $\overrightarrow{\Delta_{k}^{2} \sigma_{j, k}}$. 


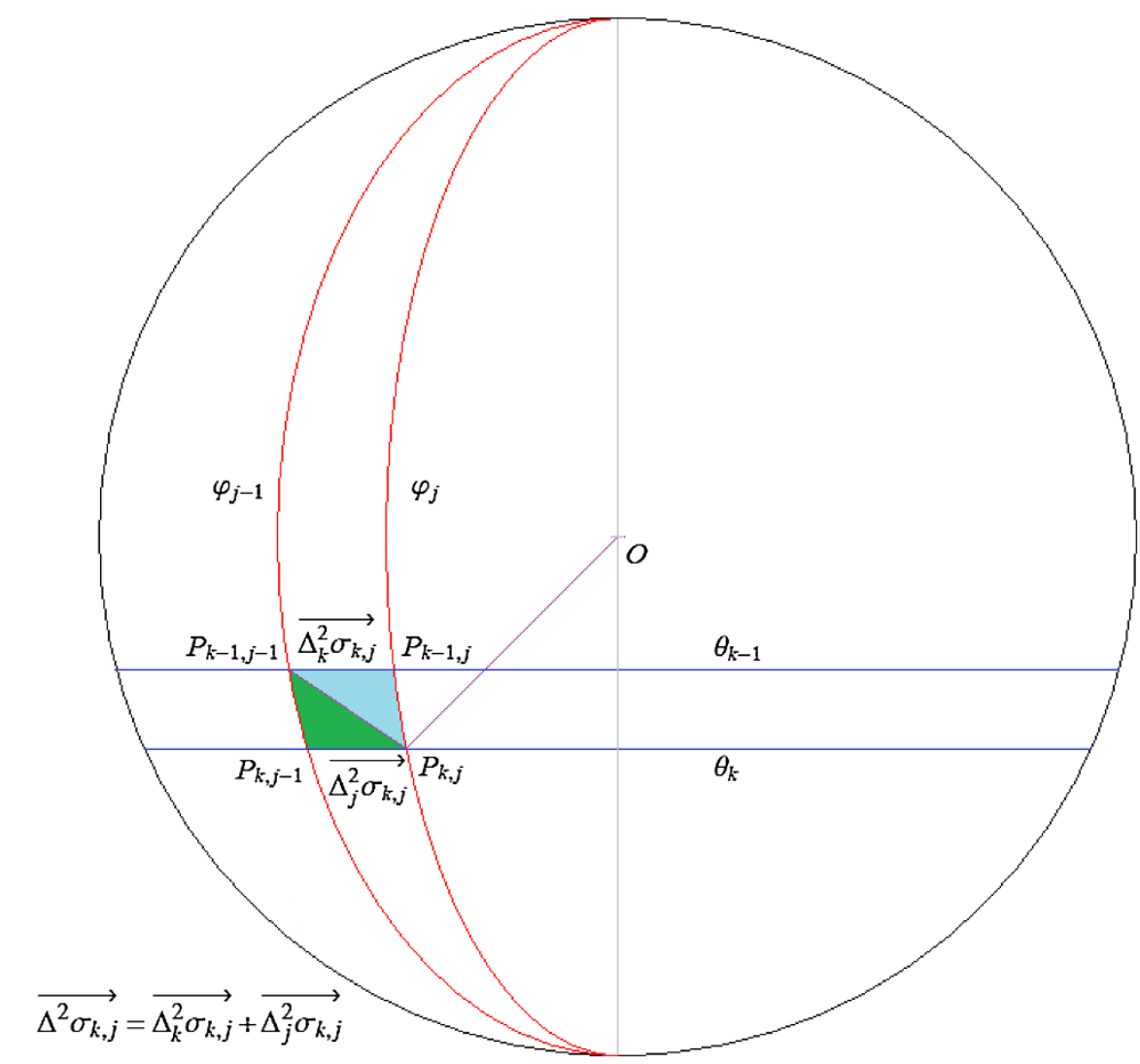

Figure 3 A lower-left piece of a divided area $\overrightarrow{\Delta^{2} \sigma_{k, j}}$. A lower-left finite area element vector $\overrightarrow{\Delta^{2} \sigma_{k j}}$ is disintegrated into two parts,

$$
\overrightarrow{\Delta^{2} \sigma_{k, j}}=\overrightarrow{\Delta_{k}^{2} \sigma_{k, j}}+\overrightarrow{\Delta_{j}^{2} \sigma_{k j}}
$$

for $j=1,2, \ldots, k$ and $k=1,2, \ldots, n$.

$\overrightarrow{\Delta_{k}^{2} \sigma_{j, k}}$ in (3.3) for $j=1,2, \ldots, k$ and $k=1,2, \ldots, n$ is expressed as

$$
\begin{aligned}
\overrightarrow{\Delta_{k}^{2} \sigma_{j, k}} & =\frac{1}{2}\left(\begin{array}{l}
x_{j-1, k-1}-x_{j, k} \\
y_{j-1, k-1}-y_{j, k} \\
z_{j-1, k-1}-z_{j, k}
\end{array}\right) \times\left(\begin{array}{l}
x_{j, k-1}-x_{j, k} \\
y_{j, k-1}-y_{j, k} \\
z_{j, k-1}-z_{j, k}
\end{array}\right) \\
& =\frac{1}{2}\left(\begin{array}{l}
y_{j, k-1}\left(z_{j, k}-z_{j-1, k-1}\right)-y_{j, k}\left(z_{j, k-1}-z_{j-1, k-1}\right)-y_{j-1, k-1}\left(z_{j, k}-z_{j, k-1}\right) \\
z_{j, k-1}\left(x_{j, k}-x_{j-1, k-1}\right)-z_{j, k}\left(x_{j, k-1}-x_{j-1, k-1}\right)-z_{j-1, k-1}\left(x_{j, k}-x_{j, k-1}\right) \\
x_{j, k-1}\left(y_{j, k}-y_{j-1, k-1}\right)-x_{j, k}\left(y_{j, k-1}-y_{j-1, k-1}\right)-x_{j-1, k-1}\left(y_{j, k}-y_{j, k-1}\right)
\end{array}\right) .
\end{aligned}
$$

We also introduce $\Delta_{k}\left(x^{\mu}\right)_{j, k}$ and $\Delta_{j}\left(x^{\mu}\right)_{j, k-1}$ for $\mu=1,2,3$ and $j=1,2, \ldots, k$ and $k=1,2, \ldots, n$ as

$$
\begin{aligned}
& \Delta_{k}\left(x^{\mu}\right)_{j, k} \equiv\left(x^{\mu}\right)_{j, k}-\left(x^{\mu}\right)_{j, k-1}, \\
& \Delta_{j}\left(x^{\mu}\right)_{j, k-1} \equiv\left(x^{\mu}\right)_{j, k-1}-\left(x^{\mu}\right)_{j-1, k-1} .
\end{aligned}
$$




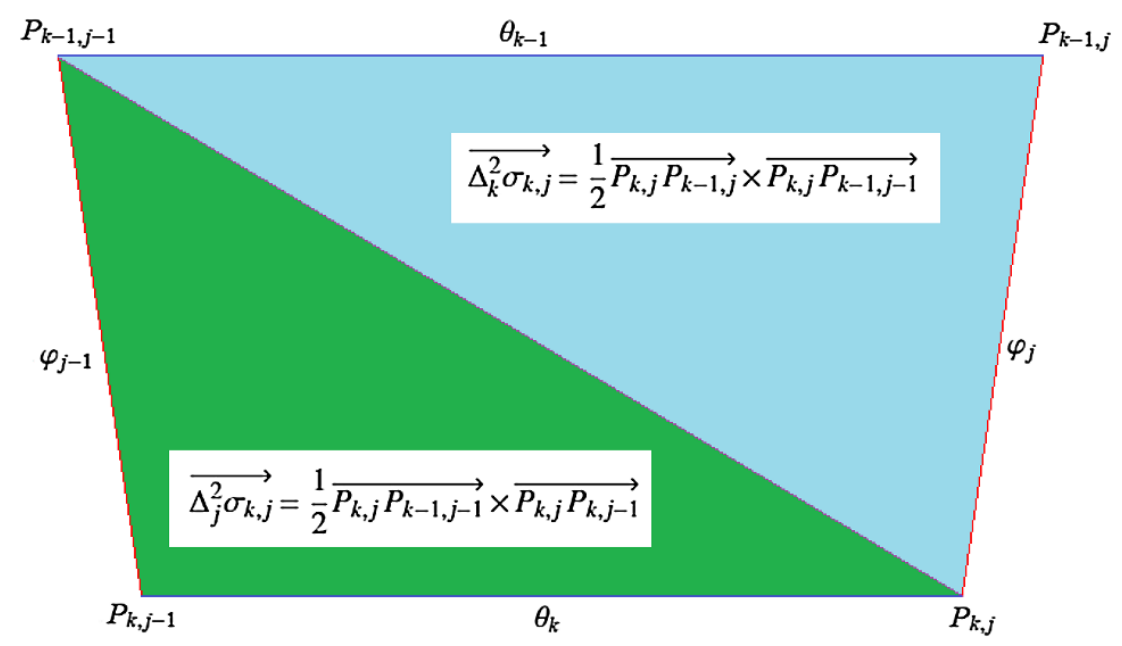

Figure 4 Two kinds of the finite area element of a lower-left piece. Two kinds of finite area element vectors $\overrightarrow{\Delta_{k}^{2} \sigma_{k, j}}$ and $\overrightarrow{\Delta_{j}^{2} \sigma_{k j}}$ in (3.4) shown in Figure 3 are introduced as

$$
\begin{array}{r}
\overrightarrow{\Delta_{k}^{2} \sigma_{k j}}=\frac{1}{2} \overrightarrow{P_{k_{j}} P_{k-1, j}} \times \overrightarrow{P_{k, j} P_{k-1, j-1}} \\
\overrightarrow{\Delta_{j}^{2} \sigma_{k j}}=\frac{1}{2} \overrightarrow{P_{k_{j}} P_{k-1, j-1}} \times \overrightarrow{P_{k j} P_{k, j-1}} \\
\text { for } j=1,2, \ldots, k \text { and } k=1,2, \ldots, n .
\end{array}
$$

The concrete forms of $\Delta_{k} x_{j, k}, \Delta_{k} y_{j, k}, \Delta_{k} z_{j, k}, \Delta_{j} x_{j, k-1}, \Delta_{j} y_{j, k-1}$ and $\Delta_{j} z_{j, k-1}$ are respectively shown in (3.50), (3.51), (3.52), (3.53), (3.54) and (3.55) in the case of the sphere. Substituting (3.14) and (3.15) into (3.13), we modify the $x$-component of $\overrightarrow{\Delta_{k}^{2} \sigma_{j, k}}$ for $j=1,2, \ldots, k$ and $k=1,2, \ldots, n$ as

$$
\begin{aligned}
\left(\overrightarrow{\Delta_{k}^{2} \sigma_{j, k}}\right)_{x} & =\frac{1}{2}\left[y_{j, k-1}\left(\Delta_{k} z_{j, k}+\Delta_{j} z_{j, k-1}\right)-y_{j, k} \Delta_{j} z_{j, k-1}-y_{j-1, k-1} \Delta_{k} z_{j, k}\right] \\
& =\frac{1}{2}\left[\left(y_{j, k-1}-y_{j-1, k-1}\right) \Delta_{k} z_{j, k}-\left(y_{j, k}-y_{j, k-1}\right) \Delta_{j} z_{j, k-1}\right] \\
& =\frac{1}{2}\left(\Delta_{k} z_{j, k} \Delta_{j} y_{j, k-1}-\Delta_{k} y_{j, k} \Delta_{j} z_{j, k-1}\right) .
\end{aligned}
$$

In the similar manner, we respectively modify the $y$ - and the $z$-components of (3.13) for $j=1,2, \ldots, k$ and $k=1,2, \ldots, n$ as

$$
\begin{aligned}
& \left(\overrightarrow{\Delta_{k}^{2} \sigma_{j, k}}\right)_{y}=\frac{1}{2}\left(\Delta_{k} x_{j, k} \Delta_{j} z_{j, k-1}-\Delta_{k} z_{j, k} \Delta_{j} x_{j, k-1}\right), \\
& \left(\overrightarrow{\Delta_{k}^{2} \sigma_{j, k}}\right)_{z}=\frac{1}{2}\left(\Delta_{k} y_{j, k} \Delta_{j} x_{j, k-1}-\Delta_{k} x_{j, k} \Delta_{j} y_{j, k-1}\right) .
\end{aligned}
$$

2. The lower-left finite area element vector $\overrightarrow{\Delta^{2} \sigma_{k, j}}$.

(a) Component representation of $\overrightarrow{\Delta_{k}^{2} \sigma_{k, j}}$. 
In the similar manner, we respectively introduce $\Delta_{k}\left(x^{\mu}\right)_{k, j}$ and $\Delta_{j}\left(x^{\mu}\right)_{k-1, j}$ for $\mu=1,2,3$ and $j=1,2, \ldots, k$ and $k=1,2, \ldots, n$ as

$$
\begin{aligned}
& \Delta_{k}\left(x^{\mu}\right)_{k, j} \equiv\left(x^{\mu}\right)_{k, j}-\left(x^{\mu}\right)_{k-1, j} \\
& \Delta_{j}\left(x^{\mu}\right)_{k-1, j} \equiv\left(x^{\mu}\right)_{k-1, j}-\left(x^{\mu}\right)_{k-1, j-1} .
\end{aligned}
$$

The concrete forms of $\Delta_{k} x_{k, j}, \Delta_{k} y_{k, j}, \Delta_{k} z_{k, j}, \Delta_{j} x_{k-1, j}, \Delta_{j} y_{k-1, j}$ and $\Delta_{j} z_{k-1, j}$ are respectively shown in (3.61), (3.62), (3.63), (3.64), (3.65) and (3.66) in the case of the sphere. Interchanging $j$ and $k$ in (3.2), we immediately obtain (3.5). In the similar manner, interchanging $j$ and $k$ in (3.10), (3.11) and (3.12) for $j=1,2, \ldots, k$ and $k=1,2, \ldots, n$, we respectively obtain

$$
\begin{aligned}
& \left(\overrightarrow{\Delta_{k}^{2} \sigma_{k, j}}\right)_{x}=\frac{1}{2}\left(\Delta_{k} y_{k, j} \Delta_{j} z_{k-1, j}-\Delta_{k} z_{k, j} \Delta_{j} y_{k-1, j}\right), \\
& \left(\overrightarrow{\Delta_{k}^{2} \sigma_{k, j}}\right)_{y}=\frac{1}{2}\left(\Delta_{k} z_{k, j} \Delta_{j} x_{k-1, j}-\Delta_{k} x_{k, j} \Delta_{j} z_{k-1, j}\right), \\
& \left(\overrightarrow{\Delta_{k}^{2} \sigma_{k, j}}\right)_{z}=\frac{1}{2}\left(\Delta_{k} x_{k, j} \Delta_{j} y_{k-1, j}-\Delta_{k} y_{k, j} \Delta_{j} x_{k-1, j}\right) .
\end{aligned}
$$

(b) Component representation of $\overrightarrow{\Delta_{j}^{2} \sigma_{k, j}}$.

In the similar manner, we respectively introduce $\Delta_{j}\left(x^{\mu}\right)_{k, j}$ and $\Delta_{k}\left(x^{\mu}\right)_{k, j-1}$ for $\mu=1,2,3$ and $j=1,2, \ldots, k$ and $k=1,2, \ldots, n$ as

$$
\begin{aligned}
& \Delta_{j}\left(x^{\mu}\right)_{k, j} \equiv\left(x^{\mu}\right)_{k, j}-\left(x^{\mu}\right)_{k, j-1}, \\
& \Delta_{k}\left(x^{\mu}\right)_{k, j-1} \equiv\left(x^{\mu}\right)_{k, j-1}-\left(x^{\mu}\right)_{k-1, j-1} .
\end{aligned}
$$

The concrete forms of $\Delta_{j} x_{k, j}, \Delta_{j} y_{k, j}, \Delta_{j} z_{k, j}, \Delta_{k} x_{k, j-1}, \Delta_{k} y_{k, j-1}$ and $\Delta_{k} z_{k, j-1}$ are respectively shown in (3.67), (3.68), (3.69), (3.70), (3.71) and (3.72) in the case of the sphere. Interchanging $j$ and $k$ in (3.3), we immediately obtain (3.6). In the similar manner, interchanging $j$ and $k$ in (3.16), (3.17) and (3.18) for $j=1,2, \ldots, k$ and $k=1,2, \ldots, n$, we respectively obtain

$$
\begin{aligned}
\left(\overrightarrow{\Delta_{j}^{2} \sigma_{k, j}}\right)_{x} & =\frac{1}{2}\left(\Delta_{j} z_{k, j} \Delta_{k} y_{k, j-1}-\Delta_{j} y_{k, j} \Delta_{k} z_{k, j-1}\right), \\
\left(\overrightarrow{\Delta_{j}^{2} \sigma_{k, j}}\right)_{y} & =\frac{1}{2}\left(\Delta_{j} x_{k, j} \Delta_{k} z_{k, j-1}-\Delta_{j} z_{k, j} \Delta_{k} x_{k, j-1}\right), \\
\left(\overrightarrow{\Delta_{j}^{2} \sigma_{k, j}}\right)_{z} & =\frac{1}{2}\left(\Delta_{j} y_{k, j} \Delta_{k} x_{k, j-1}-\Delta_{j} x_{k, j} \Delta_{k} y_{k, j-1}\right) .
\end{aligned}
$$

\subsection{The sum of surface areas consisting of triangles}

Let us divide the area of the 2D surface of a 3D solid into $n^{2}$ areas, i.e., $n$-division for longitudinal direction and $n$-division for latitudinal direction. The longitudinal and latitudinal directions of the surface are divided by the same number $n$ for a single limit. The sum of divided areas is expressed as $S_{n^{2}}$. It is separated into two parts, one is the upper-right part and the other is the lower-left part. The diagonal components are subtracted from the sum of the upper-right and the lower-left parts since they are overlapped. 
Proposition 1 Let $S_{n^{2}}$ be a sum of divided areas of the $2 D$ surface of a $3 D$ solid. $S_{n^{2}}$ is expressed as

$$
S_{n^{2}}=\sum_{k=1}^{n} \sum_{j=1}^{k}\left(\overrightarrow{n_{j, k}} \cdot \overrightarrow{\Delta^{2} \sigma_{j, k}}+\overrightarrow{n_{k, j}} \cdot \overrightarrow{\Delta^{2} \sigma_{k, j}}\right)-\sum_{k=1}^{n} \overrightarrow{n_{k, k}} \cdot \overrightarrow{\Delta^{2} \sigma_{k, k}},
$$

where the upper-right finite area element vector $\overrightarrow{\Delta^{2} \sigma_{j, k}}$ and the lower-left one $\overrightarrow{\Delta^{2} \sigma_{k, j}}$ are divided areas of the $2 D$ surface and $\overrightarrow{n_{j, k}}$ and $\overrightarrow{n_{k, j}}$ are corresponding unit normal vectors of respective divided areas.

Proof $S_{n^{2}}$ is introduced as

$$
\begin{aligned}
S_{n^{2}}= & \overrightarrow{n_{1,1}} \cdot \overrightarrow{\Delta^{2} \sigma_{1,1}}+\overrightarrow{n_{1,2}} \cdot \overrightarrow{\Delta^{2} \sigma_{1,2}}+\overrightarrow{n_{1,3}} \cdot \overrightarrow{\Delta^{2} \sigma_{1,3}}+\cdots+\overrightarrow{n_{1, n}} \cdot \overrightarrow{\Delta^{2} \sigma_{1, n}} \\
& +\overrightarrow{n_{2,1}} \cdot \overrightarrow{\Delta^{2} \sigma_{2,1}}+\overrightarrow{n_{2,2}} \cdot \overrightarrow{\Delta^{2} \sigma_{2,2}}+\overrightarrow{n_{2,3}} \cdot \overrightarrow{\Delta^{2} \sigma_{2,3}}+\cdots+\overrightarrow{n_{2, n}} \cdot \overrightarrow{\Delta^{2} \sigma_{2, n}} \\
& +\overrightarrow{n_{3,1}} \cdot \overrightarrow{\Delta^{2} \sigma_{3,1}}+\overrightarrow{n_{3,2}} \cdot \overrightarrow{\Delta^{2} \sigma_{3,2}}+\overrightarrow{n_{3,3}} \cdot \overrightarrow{\Delta^{2} \sigma_{3,3}}+\cdots+\overrightarrow{n_{3, n}} \cdot \overrightarrow{\Delta^{2} \sigma_{3, n}} \\
& +\overrightarrow{n_{n, 1}} \cdot \overrightarrow{\Delta^{2} \sigma_{n, 1}}+\overrightarrow{n_{n, 2}} \cdot \overrightarrow{\Delta^{2} \sigma_{n, 2}}+\overrightarrow{n_{n, 3}} \cdot \overrightarrow{\Delta^{2} \sigma_{n, 3}}+\cdots+\overrightarrow{n_{n, n}} \cdot \overrightarrow{\Delta^{2} \sigma_{n, n}}
\end{aligned}
$$

As shown in Figures 5 and 6, the upper-right components of $S_{n^{2}}$ in (3.30), including the diagonal ones, are expressed as

$$
\begin{array}{r}
\sum_{k=1}^{n} \sum_{j=1}^{k} \overrightarrow{n_{j, k}} \cdot \overrightarrow{\Delta^{2} \sigma_{j, k}} \\
=\overrightarrow{n_{1,1}} \cdot \overrightarrow{\Delta^{2} \sigma_{1,1}}+\overrightarrow{n_{1,2}} \cdot \overrightarrow{\Delta^{2} \sigma_{1,2}}+\overrightarrow{n_{1,3}} \cdot \overrightarrow{\Delta^{2} \sigma_{1,3}}+\cdots+\overrightarrow{n_{1, n}} \cdot \overrightarrow{\Delta^{2} \sigma_{1, n}} \\
+\overrightarrow{n_{2,2}} \cdot \overrightarrow{\Delta^{2} \sigma_{2,2}}+\overrightarrow{n_{2,3}} \cdot \overrightarrow{\Delta^{2} \sigma_{2,3}}+\cdots+\overrightarrow{n_{2, n}} \cdot \overrightarrow{\Delta^{2} \sigma_{2, n}} \\
+\overrightarrow{n_{3,3}} \cdot \overrightarrow{\Delta^{2} \sigma_{3,3}}+\cdots+\overrightarrow{n_{3, n}} \cdot \overrightarrow{\Delta^{2} \sigma_{3, n}} \\
\ddots \\
+\overrightarrow{n_{n, n}} \cdot \overrightarrow{\Delta^{2} \sigma_{n, n}} .
\end{array}
$$

As shown in Figures 7 and 8, the lower-left components of $S_{n^{2}}$ in (3.30), including the diagonal ones, are expressed as

$$
\begin{aligned}
& \sum_{k=1}^{n} \sum_{j=1}^{k} \overrightarrow{n_{k, j}} \cdot \overrightarrow{\Delta^{2} \sigma_{k, j}} \\
& =\overrightarrow{n_{1,1}} \cdot \overrightarrow{\Delta^{2} \sigma_{1,1}} \\
& \quad+\overrightarrow{n_{2,1}} \cdot \overrightarrow{\Delta^{2} \sigma_{2,1}}+\overrightarrow{n_{2,2}} \cdot \overrightarrow{\Delta^{2} \sigma_{2,2}} \\
& \quad+\overrightarrow{n_{3,1}} \cdot \overrightarrow{\Delta^{2} \sigma_{3,1}}+\overrightarrow{n_{3,2}} \cdot \overrightarrow{\Delta^{2} \sigma_{3,2}}+\overrightarrow{n_{3,3}} \cdot \overrightarrow{\Delta^{2} \sigma_{3,3}} \\
& \quad \quad \vdots \\
& \quad+\overrightarrow{n_{n, 1}} \cdot \overrightarrow{\Delta^{2} \sigma_{n, 1}}+\overrightarrow{n_{n, 2}} \cdot \overrightarrow{\Delta^{2} \sigma_{n, 2}}+\overrightarrow{n_{n, 3}} \cdot \overrightarrow{\Delta^{2} \sigma_{n, 3}}+\cdots+\overrightarrow{n_{n, n}} \cdot \overrightarrow{\Delta^{2} \sigma_{n, n}}
\end{aligned}
$$




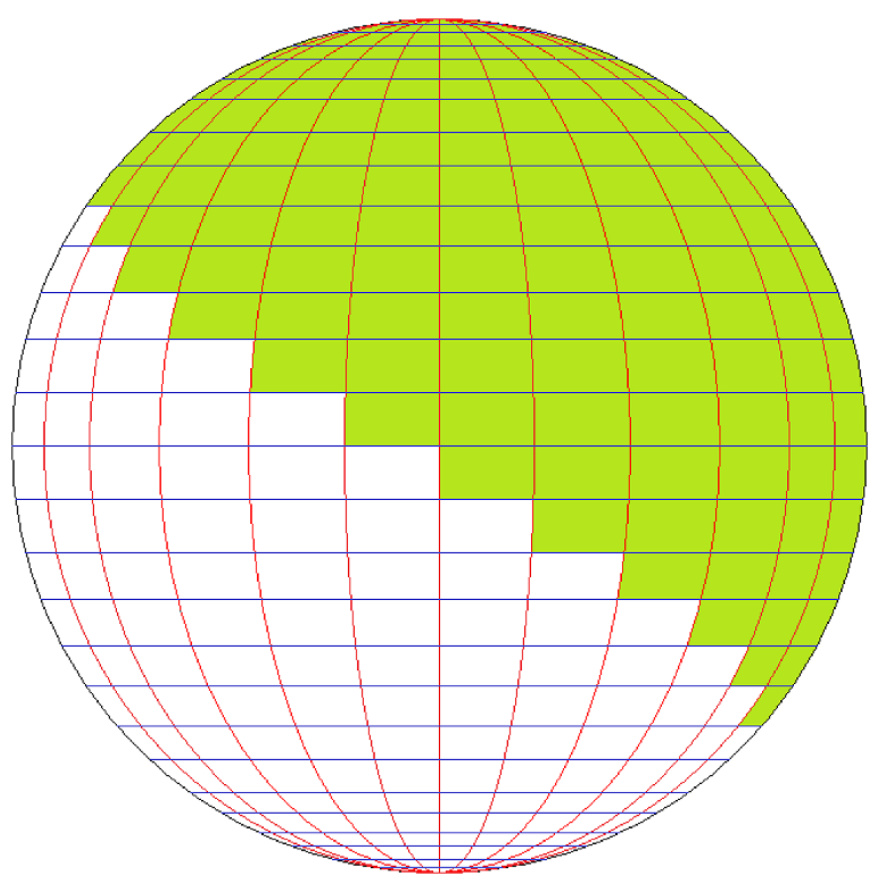

Figure 5 The upper-right pieces of a divided area on the surface of the sphere. The sum of the upper-right pieces of a divided area on the surface of the sphere is

$$
\begin{aligned}
& \sum_{k=1}^{n} \sum_{j=1}^{k} \overrightarrow{n_{j, k}} \cdot \overrightarrow{\Delta^{2} \sigma_{j, k}}=\overrightarrow{n_{1,1}} \cdot \overrightarrow{\Delta^{2} \sigma_{1,1}}+\overrightarrow{n_{1,2}} \cdot \overrightarrow{\Delta^{2} \sigma_{1,2}} \\
&+\overrightarrow{n_{1,3}} \cdot \overrightarrow{\Delta^{2} \sigma_{1,3}}+\cdots+\overrightarrow{n_{1, n}} \cdot \overrightarrow{\Delta^{2} \sigma_{1, n}} \\
&+\overrightarrow{n_{2,2}} \cdot \overrightarrow{\Delta^{2} \sigma_{2,2}}+\overrightarrow{n_{2,3}} \cdot \overrightarrow{\Delta^{2} \sigma_{2,3}}+\cdots+\overrightarrow{n_{2, n}} \cdot \overrightarrow{\Delta^{2} \sigma_{2, n}} \\
&+\overrightarrow{n_{3,3}} \cdot \overrightarrow{\Delta^{2} \sigma_{3,3}}+\cdots+\overrightarrow{n_{3, n}} \cdot \overrightarrow{\Delta^{2} \sigma_{3, n}}
\end{aligned}
$$

$$
+\overrightarrow{n_{n, n}} \cdot \overrightarrow{\Delta^{2} \sigma_{n, n}}
$$

Combining (3.31) with (3.32) and subtracting the diagonal components, we obtain (3.29).

Q.E.D.

The area $S$ of the surface of the 3D solid is defined as the limit of $S_{n^{2}}$ at infinity $n \rightarrow \infty$, i.e.,

$$
S=\lim _{n \rightarrow \infty} S_{n^{2}}
$$

Proposition 2 The area $S$ of the $2 D$ surface of the $3 D$ solid in $\mathbb{R}^{3}$ is expressed as

$$
S=\lim _{n \rightarrow \infty} \sum_{k=1}^{n} \sum_{j=1}^{k}\left(\overrightarrow{n_{j, k}} \cdot \overrightarrow{\Delta^{2} \sigma_{j, k}}+\overrightarrow{n_{k, j}} \cdot \overrightarrow{\Delta^{2} \sigma_{k, j}}\right)
$$




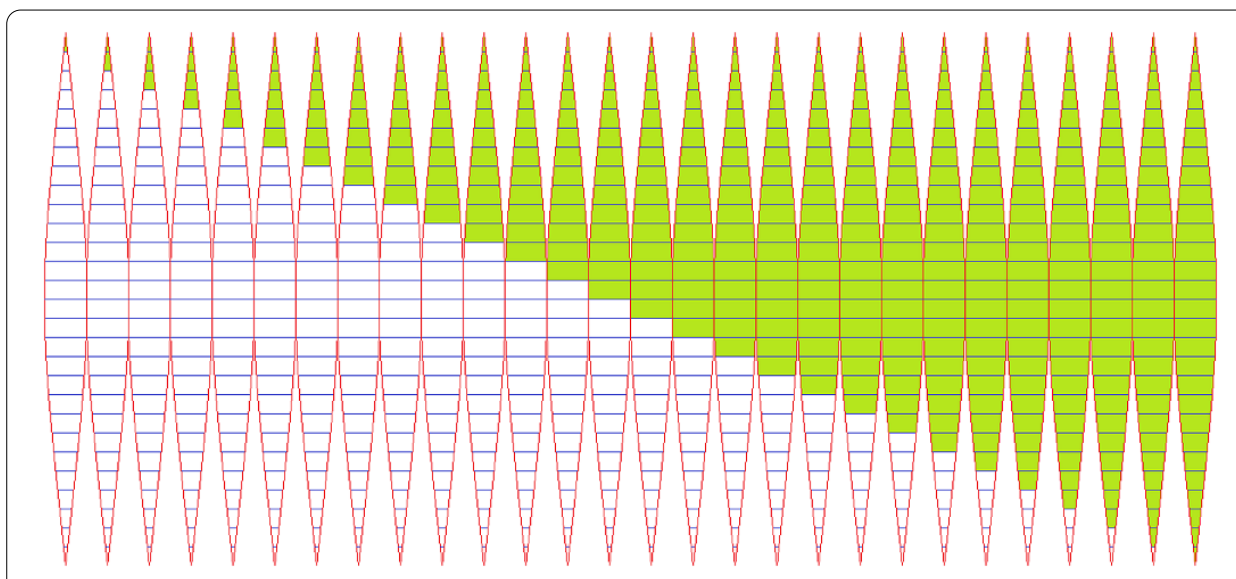

Figure 6 Expanded form of the upper-right pieces of a divided area. This is an expanded form of the upper-right piece of a divided area of the surface of the sphere shown in Figure 5.

under the condition of

$$
\lim _{n \rightarrow \infty} \sum_{k=1}^{n} \overrightarrow{n_{k, k}} \cdot \overrightarrow{\Delta^{2} \sigma_{k, k}}=0,
$$

where the upper-right finite area element vector $\overrightarrow{\Delta^{2} \sigma_{j, k}}$ and the lower-left one $\overrightarrow{\Delta^{2} \sigma_{k, j}}$ are divided areas of the $2 D$ surface and $\overrightarrow{n_{j, k}}$ and $\overrightarrow{n_{k, j}}$ are corresponding unit normal vectors of respective divided areas.

Proof Substituting (3.29) into (3.33), we obtain a formula of the total area $S$ as (3.34) under the condition of (3.35).

Q.E.D.

\subsection{Surface area of the sphere}

The Cartesian coordinates $(x, y, z)$ are transformed into the spherical coordinates $(\varphi, \theta, r)$ as

$$
\begin{aligned}
& x=r \sin \theta \cos \varphi, \\
& y=r \sin \theta \sin \varphi, \\
& z=r \cos \theta .
\end{aligned}
$$

The simple case of a sphere is considered, for example, $r=a$, where $a=$ constant. A kind of spherical sequence is introduced in order to sweep all the surfaces of the sphere. The following two sets of notations, $\left(\theta_{j}, \varphi_{k}\right)$ for the upper-right finite area element vector and $\left(\theta_{k}, \varphi_{j}\right)$ for the lower-left one are introduced here.

1. The upper-right finite area element vector $\overrightarrow{\Delta^{2} \sigma_{j, k}}$, expressed in terms of $\left(\theta_{j}, \varphi_{k}\right)$ for $j=0,1,2, \ldots, k$ and $k=0,1,2, \ldots, n$.

The normal vector is a unit vector $\overrightarrow{n_{j, k}}$, the direction of which is the same as that from the original point to a point on the surface in the case of the sphere,

$$
\overrightarrow{n_{j, k}}=\frac{\overrightarrow{O P_{j, k}}}{\left\|\overrightarrow{O P_{j, k}}\right\|}
$$




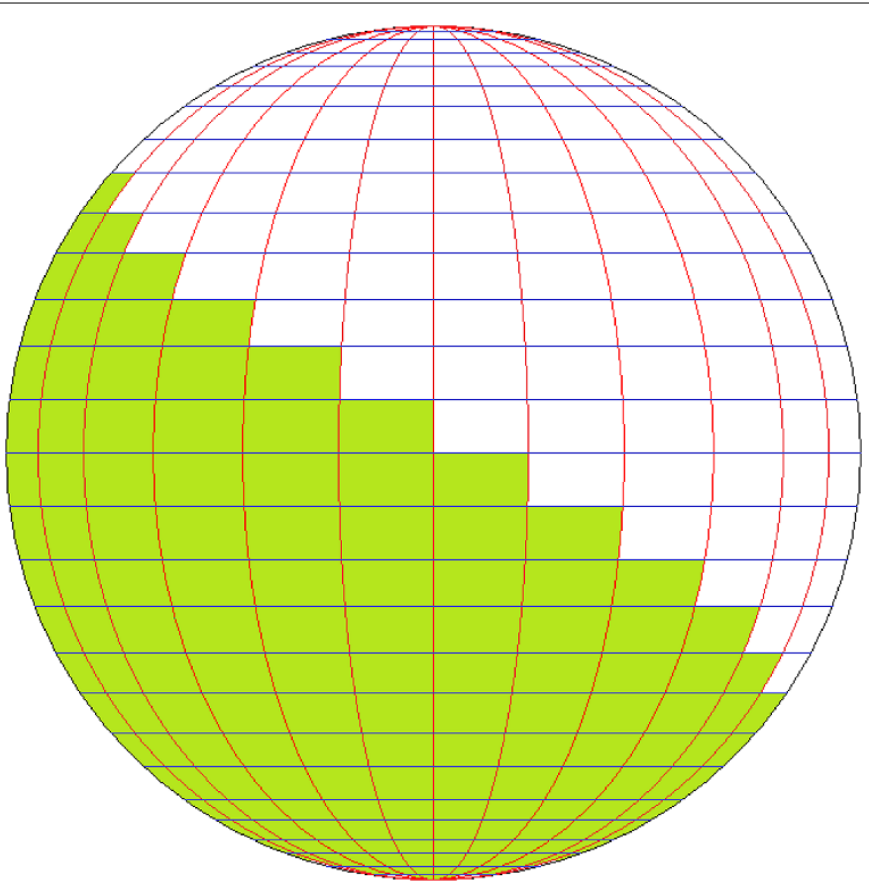

Figure 7 The lower-left pieces of a divided area on the surface of the sphere. The sum of the lower-left pieces of a divided area on the surface of the sphere is

$$
\begin{aligned}
\sum_{k=1}^{n} \sum_{j=1}^{k} \overrightarrow{n_{k j}} \cdot \overrightarrow{\Delta^{2} \sigma_{k, j}}= & \overrightarrow{n_{1,1}} \cdot \overrightarrow{\Delta^{2} \sigma_{1,1}} \\
& +\overrightarrow{n_{2,1}} \cdot \overrightarrow{\Delta^{2} \sigma_{2,1}}+\overrightarrow{n_{2,2}} \cdot \overrightarrow{\Delta^{2} \sigma_{2,2}} \\
& +\overrightarrow{n_{3,1}} \cdot \overrightarrow{\Delta^{2} \sigma_{3,1}}+\overrightarrow{n_{3,2}} \cdot \overrightarrow{\Delta^{2} \sigma_{3,2}}+\overrightarrow{n_{3,3}} \cdot \overrightarrow{\Delta^{2} \sigma_{3,3}} \\
& \vdots \\
& +\overrightarrow{n_{n, 1}} \cdot \overrightarrow{\Delta^{2} \sigma_{n, 1}}+\overrightarrow{n_{n, 2}} \cdot \overrightarrow{\Delta^{2} \sigma_{n, 2}}+\overrightarrow{n_{n, 3}} \cdot \overrightarrow{\Delta^{2} \sigma_{n, 3}}+\cdots+\overrightarrow{n_{n, n}} \cdot \overrightarrow{\Delta^{2} \sigma_{n, n}}
\end{aligned}
$$

for $j=0,1,2, \ldots, k$ and $k=0,1,2, \ldots, n$, as shown in Figure 1. In the case of $\left\|\overrightarrow{O P_{j, k}}\right\|=a, \overrightarrow{n_{j, k}}$ has the following components of

$$
\overrightarrow{n_{j, k}}=\frac{1}{a} \overrightarrow{O P_{j, k}}=\frac{1}{a}\left(\begin{array}{l}
x_{j, k} \\
y_{j, k} \\
z_{j, k}
\end{array}\right)=\frac{1}{a}\left[\left(x^{\mu}\right)_{j, k}\right]
$$

for $\mu=1,2,3$ and $j=0,1,2, \ldots, k$ and $k=0,1,2, \ldots, n$. The Cartesian coordinates of the upper-right finite area element vector $\left(\theta_{j}, \varphi_{k}\right)$ for $j=0,1,2, \ldots, k$ and $k=0,1,2, \ldots, n$ are written as

$$
\begin{aligned}
x_{j, k} & =a \sin \theta_{j} \cos \varphi_{k}, \\
y_{j, k} & =a \sin \theta_{j} \sin \varphi_{k}, \\
z_{j, k} & =a \cos \theta_{j} .
\end{aligned}
$$




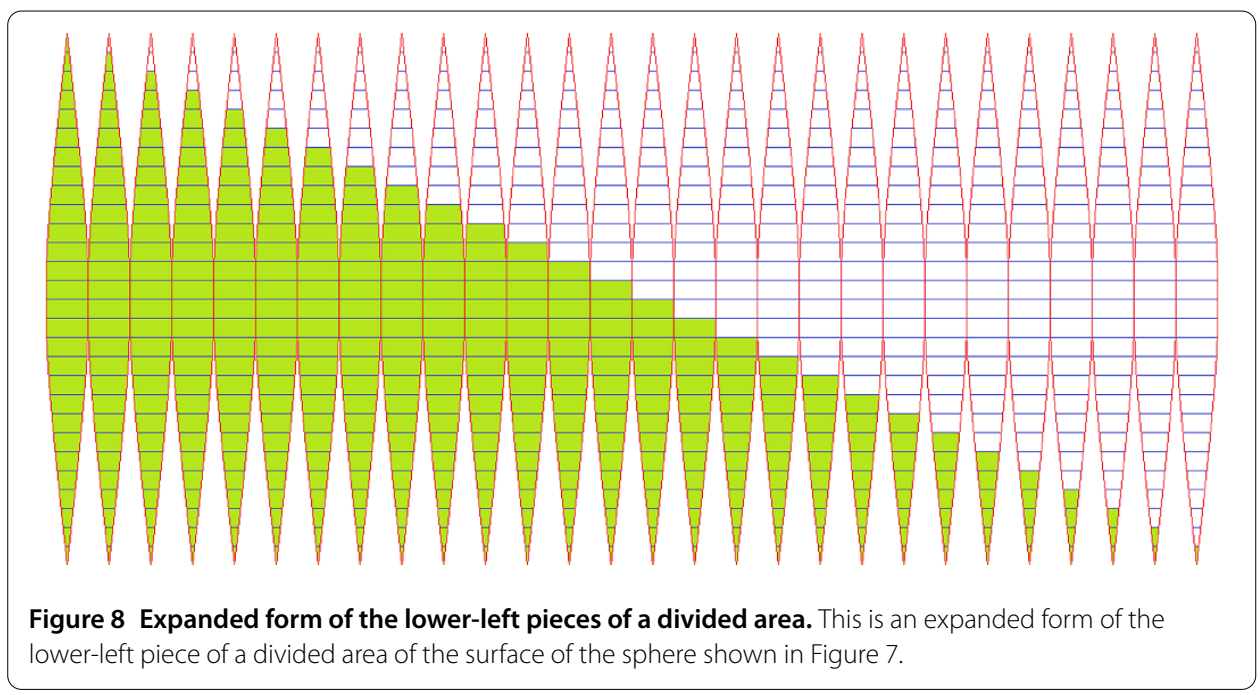

(a) Applying (3.41), (3.42) and (3.43) to (3.8) for $\mu=1,2,3$, respectively, we obtain

$$
\begin{aligned}
\Delta_{j} x_{j, k} & =a\left(\sin \theta_{j}-\sin \theta_{j-1}\right) \cos \varphi_{k}, \\
\Delta_{j} y_{j, k} & =a\left(\sin \theta_{j}-\sin \theta_{j-1}\right) \sin \varphi_{k}, \\
\Delta_{j} z_{j, k} & =a\left(\cos \theta_{j}-\cos \theta_{j-1}\right) .
\end{aligned}
$$

(b) Applying (3.41), (3.42) and (3.43) to (3.9) for $\mu=1,2,3$, respectively, we obtain

$$
\begin{aligned}
& \Delta_{k} x_{j-1, k}=a \sin \theta_{j-1}\left(\cos \varphi_{k}-\cos \varphi_{k-1}\right), \\
& \Delta_{k} y_{j-1, k}=a \sin \theta_{j-1}\left(\sin \varphi_{k}-\sin \varphi_{k-1}\right), \\
& \Delta_{k} z_{j-1, k}=0 .
\end{aligned}
$$

(c) Applying (3.41), (3.42) and (3.43) to (3.14) for $\mu=1,2,3$, respectively, we obtain

$$
\begin{aligned}
& \Delta_{k} x_{j, k}=a \sin \theta_{j}\left(\cos \varphi_{k}-\cos \varphi_{k-1}\right), \\
& \Delta_{k} y_{j, k}=a \sin \theta_{j}\left(\sin \varphi_{k}-\sin \varphi_{k-1}\right), \\
& \Delta_{k} z_{j, k}=0 .
\end{aligned}
$$

(d) Applying (3.41), (3.42) and (3.43) to (3.15) for $\mu=1,2,3$, respectively, we obtain

$$
\begin{aligned}
\Delta_{j} x_{j, k-1} & =a\left(\sin \theta_{j}-\sin \theta_{j-1}\right) \cos \varphi_{k-1}, \\
\Delta_{j} y_{j, k-1} & =a\left(\sin \theta_{j}-\sin \theta_{j-1}\right) \sin \varphi_{k-1}, \\
\Delta_{j} z_{j, k-1} & =a\left(\cos \theta_{j}-\cos \theta_{j-1}\right) .
\end{aligned}
$$

2. The lower-left finite area element vector $\overrightarrow{\Delta^{2} \sigma_{k, j}}$, expressed in terms of $\left(\theta_{k}, \varphi_{j}\right)$ for $j=$ $0,1,2, \ldots, k$ and $k=0,1,2, \ldots, n$. 
The normal vector is a unit vector $\overrightarrow{n_{k, j}}$, the direction of which is the same as that from the original point to a point on the surface in the case of the sphere,

$$
\overrightarrow{n_{k, j}}=\frac{\overrightarrow{O P_{k, j}}}{\left\|\overrightarrow{O P_{k, j}}\right\|}
$$

for $j=0,1,2, \ldots, k$ and $k=0,1,2, \ldots, n$, as shown in Figure 3. In the case of $\left\|\overrightarrow{O P_{k, j}}\right\|=a, \overrightarrow{n_{k, j}}$ has the following components of

$$
\overrightarrow{n_{k, j}}=\frac{1}{a} \overrightarrow{O P_{k, j}}=\frac{1}{a}\left(\begin{array}{l}
x_{k, j} \\
y_{k, j} \\
z_{k, j}
\end{array}\right)=\frac{1}{a}\left[\left(x^{\mu}\right)_{k, j}\right]
$$

for $\mu=1,2,3$ and $j=0,1,2, \ldots, k$ and $k=0,1,2, \ldots, n$. The Cartesian coordinates of the lower-left finite area element vectors $\left(\theta_{k}, \varphi_{j}\right)$ for $j=0,1,2, \ldots, k$ and $k=0,1,2, \ldots, n$ are written as

$$
\begin{aligned}
x_{k, j} & =a \sin \theta_{k} \cos \varphi_{j}, \\
y_{k, j} & =a \sin \theta_{k} \sin \varphi_{j}, \\
z_{k, j} & =a \cos \theta_{k} .
\end{aligned}
$$

(a) Applying (3.58), (3.59) and (3.60) to (3.19) for $\mu=1,2,3$, respectively, we obtain

$$
\begin{aligned}
& \Delta_{k} x_{k, j}=a\left(\sin \theta_{k}-\sin \theta_{k-1}\right) \cos \varphi_{j}, \\
& \Delta_{k} y_{k, j}=a\left(\sin \theta_{k}-\sin \theta_{k-1}\right) \sin \varphi_{j}, \\
& \Delta_{k} z_{k, j}=a\left(\cos \theta_{k}-\cos \theta_{k-1}\right) .
\end{aligned}
$$

(b) Applying (3.58), (3.59) and (3.60) to (3.20) for $\mu=1,2,3$, respectively, we obtain

$$
\begin{aligned}
& \Delta_{j} x_{k-1, j}=a \sin \theta_{k-1}\left(\cos \varphi_{j}-\cos \varphi_{j-1}\right), \\
& \Delta_{j} y_{k-1, j}=a \sin \theta_{k-1}\left(\sin \varphi_{j}-\sin \varphi_{j-1}\right), \\
& \Delta_{j} z_{k-1, j}=0 .
\end{aligned}
$$

(c) Applying (3.58), (3.59) and (3.60) to (3.24) for $\mu=1,2,3$, respectively, we obtain

$$
\begin{aligned}
& \Delta_{j} x_{k, j}=a \sin \theta_{k}\left(\cos \varphi_{j}-\cos \varphi_{j-1}\right), \\
& \Delta_{j} y_{k, j}=a \sin \theta_{k}\left(\sin \varphi_{j}-\sin \varphi_{j-1}\right), \\
& \Delta_{j} z_{k, j}=0 .
\end{aligned}
$$

(d) Applying (3.58), (3.59) and (3.60) to (3.25) for $\mu=1,2,3$, respectively, we obtain

$$
\begin{aligned}
& \Delta_{k} x_{k, j-1}=a\left(\sin \theta_{k}-\sin \theta_{k-1}\right) \cos \varphi_{j-1}, \\
& \Delta_{k} y_{k, j-1}=a\left(\sin \theta_{k}-\sin \theta_{k-1}\right) \sin \varphi_{j-1}, \\
& \Delta_{k} z_{k, j-1}=a\left(\cos \theta_{k}-\cos \theta_{k-1}\right) .
\end{aligned}
$$


Example 1 The domain of $\theta$ is $0 \leq \theta \leq \pi$ and that of $\varphi$ is $0 \leq \varphi \leq 2 \pi$. The surface area of the sphere is divided into two parts as follows.

1. For the upper-right finite area element vector, $\theta_{j}$ and $\varphi_{k}$ are respectively introduced as

$$
\begin{aligned}
& \theta_{j}=\frac{j}{n} \pi, \\
& \varphi_{k}=2 \frac{k}{n} \pi
\end{aligned}
$$

for $j=0,1,2, \ldots, k$ and $k=0,1,2, \ldots, n$, as shown in Figure 2. The concrete increments are shown in the first part of Appendix A. Using (3.10), (3.11), (3.12), (3.16), (3.17) and (3.18), we obtain the upper-right area as

$$
\lim _{n \rightarrow \infty} \sum_{k=1}^{n} \sum_{j=1}^{k} \overrightarrow{n_{j, k}} \cdot\left(\overrightarrow{\Delta_{j}^{2} \sigma_{j, k}}+\overrightarrow{\Delta_{k}^{2} \sigma_{j, k}}\right)=2 \pi a^{2} .
$$

The calculations in detail are shown in the first part of Appendix B in the case of $b=1$.

2. For the lower-left finite area element vector, $\theta_{k}$ and $\varphi_{j}$ are respectively introduced as

$$
\begin{aligned}
\theta_{k} & =\frac{k}{n} \pi, \\
\varphi_{j} & =2 \frac{j}{n} \pi
\end{aligned}
$$

for $j=0,1,2, \ldots, k$ and $k=0,1,2, \ldots, n$, as shown in Figure 4 . The concrete increments are shown in the second part of Appendix A. Using (3.21), (3.22), (3.23), (3.26), (3.27) and (3.28), we obtain the lower-left area as

$$
\lim _{n \rightarrow \infty} \sum_{k=1}^{n} \sum_{j=1}^{k} \overrightarrow{n_{k, j}} \cdot\left(\overrightarrow{\Delta_{k}^{2} \sigma_{k, j}}+\overrightarrow{\Delta_{j}^{2} \sigma_{k, j}}\right)=2 \pi a^{2} .
$$

The calculations in detail are shown in the second part of Appendix B in the case of $b=1$.

Substituting (3.75) and (3.78) into (3.34), we obtain the surface area of the sphere as

$$
\begin{aligned}
S & =\lim _{n \rightarrow \infty} \sum_{k=1}^{n} \sum_{j=1}^{k}\left(\overrightarrow{n_{j, k}} \cdot \overrightarrow{\Delta^{2} \sigma_{j, k}}+\overrightarrow{n_{k, j}} \cdot \overrightarrow{\Delta^{2} \sigma_{k, j}}\right) \\
& =4 \pi a^{2} .
\end{aligned}
$$

We thus obtained the value of the surface area of the sphere by calculating it as the sum of triangles. This calculation is the limit at infinity $n \rightarrow \infty$ of the finite element method. Using formula manipulation software, the approximate values according to the finite element method are calculated in Table 1 and their graph is plotted in Figure 9. 
Table 1 Representative approximate values of the surface areas of the sphere

\begin{tabular}{|c|c|c|c|}
\hline$n$ & $\frac{1}{a^{2}} \sum_{k=1}^{n} \sum_{j=1}^{k}\left(\overrightarrow{n_{j, k}} \cdot \overrightarrow{\Delta^{2} \sigma_{j, k}}+\overrightarrow{n_{k, j}} \cdot \overrightarrow{\Delta^{2} \sigma_{k, j}}\right)$ & $\frac{1}{a^{2}} \sum_{k=1}^{n} \overrightarrow{n_{k, k}} \cdot \overrightarrow{\Delta^{2} \sigma_{k, k}}$ & $\frac{1}{a^{2}} S_{n^{2}}$ \\
\hline 4 & $8.53551 \ldots$ & $1.70709 \ldots$ & $6.82842 \ldots$ \\
\hline 8 & $12.24348 \ldots$ & $1.36038 \ldots$ & $10.88310 \ldots$ \\
\hline 16 & $12.88623 \ldots$ & $0.75801 \ldots$ & $12.12819 \ldots$ \\
\hline 32 & $12.84477 \ldots$ & $0.38922 \ldots$ & $12.45552 \ldots$ \\
\hline 64 & $12.73995 \ldots$ & $0.19590 \ldots$ & $12.54405 \ldots$ \\
\hline 128 & $12.65754 \ldots$ & $0.09810 \ldots$ & $12.55941 \ldots$ \\
\hline 256 & $12.61371 \ldots$ & $0.04908 \ldots$ & $12.56463 \ldots$ \\
\hline 512 & $12.59052 \ldots$ & $0.02454 \ldots$ & $12.56598 \ldots$ \\
\hline \multirow[t]{2}{*}{1024} & $12.57852 \ldots$ & $0.01227 \ldots$ & $12.56625 \ldots$ \\
\hline & & . & \\
\hline$\infty$ & $12.56637 \ldots$ & 0 & $12.56637 \ldots$ \\
\hline
\end{tabular}

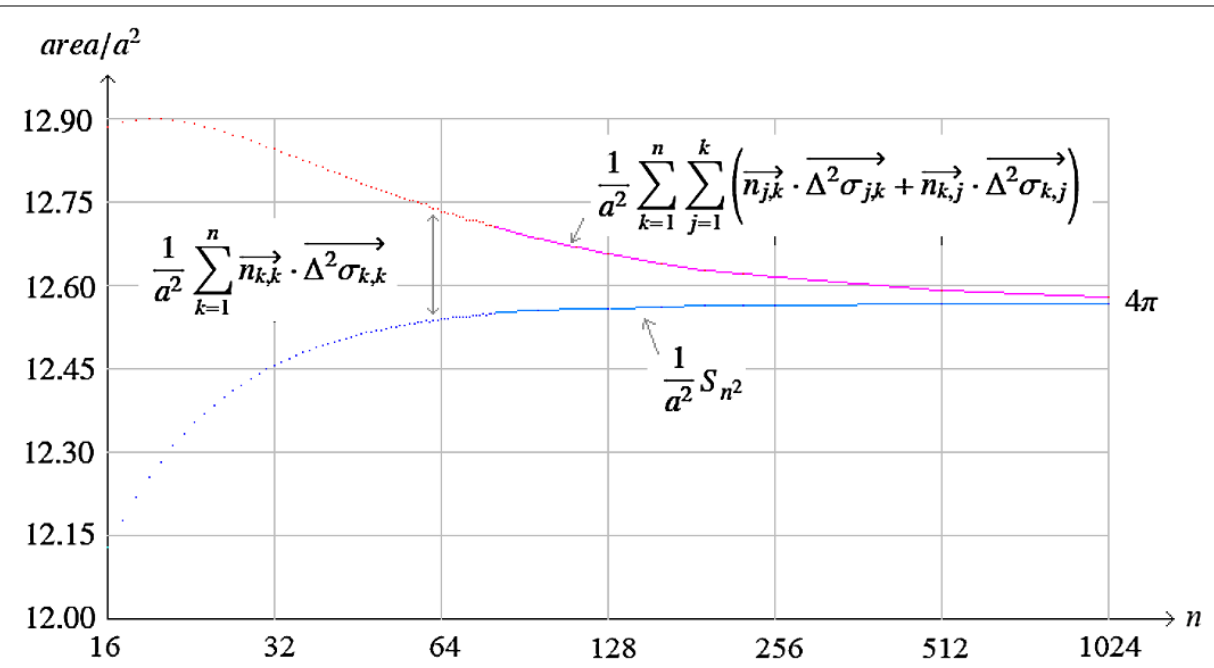

Figure 9 Convergence of the two kinds of approximate values of the surface areas of the sphere. Let $S_{n^{2}}$ be a sum of divided areas of the $2 D$ surface of a $3 D$ solid. $S_{n^{2}}$ is expressed as

$$
S_{n^{2}}=\sum_{k=1}^{n} \sum_{j=1}^{k}\left(\overrightarrow{n_{j, k}} \cdot \overrightarrow{\Delta^{2} \sigma_{j, k}}+\overrightarrow{n_{k j}} \cdot \overrightarrow{\Delta^{2} \sigma_{k, j}}\right)-\sum_{k=1}^{n} \overrightarrow{n_{k, k}} \cdot \overrightarrow{\Delta^{2} \sigma_{k, k}}
$$

where two kinds of finite area element vectors $\overrightarrow{\Delta^{2} \sigma_{j, k}}$ and $\overrightarrow{\Delta^{2} \sigma_{k, j}}$ are divided areas of the $2 \mathrm{D}$ surface and $\overrightarrow{n_{j, k}}$ and $\vec{n}_{k, j}$ are corresponding unit normal vectors of respective divided area elements. As

$\sum_{k=1}^{n} \overrightarrow{n_{k, k}} \cdot \overrightarrow{\Delta^{2} \sigma_{k, k}} \rightarrow 0$,

$$
\sum_{k=1}^{n} \sum_{j=1}^{k}\left(\overrightarrow{n_{j, k}} \cdot \overrightarrow{\Delta^{2} \sigma_{j, k}}+\overrightarrow{n_{k, j}} \cdot \overrightarrow{\Delta^{2} \sigma_{k, j}}\right)
$$




\subsection{Two kinds of finite area element vectors in the 3D space in tensor representation}

Before expressing the following formulae in tensor representation, the antisymmetric symbol in the 3D space is introduced as

$$
\varepsilon^{\alpha \beta \gamma}=\varepsilon_{\alpha \beta \gamma}= \begin{cases}+1 & \text { for even permutation of }\{1,2,3\}, \\ -1 & \text { for odd permutation of }\{1,2,3\}, \\ 0 & \text { otherwise. }\end{cases}
$$

In the 3D space, $\frac{1}{2} \varepsilon_{\alpha \beta \mu} \varepsilon^{\alpha \beta \gamma}=\delta_{\mu}^{\gamma}$ holds for $\gamma, \mu=1,2,3$, where the indices are summed over $\alpha, \beta=1,2,3 . \frac{1}{3 !} \varepsilon_{\alpha \beta \gamma} \varepsilon^{\alpha \beta \gamma}=1$ also holds, where the indices are summed over $\alpha, \beta, \gamma=1,2,3$.

1. The upper-right finite area element vector $\left(\Delta^{2} \sigma_{\mu}\right)_{j, k} \equiv\left(\overrightarrow{\Delta^{2} \sigma_{j, k}}\right)_{\mu}$ for $\mu=1,2,3$ and $j=1,2, \ldots, k$ and $k=1,2, \ldots, n$ is disintegrated into

$$
\left(\overrightarrow{\Delta^{2} \sigma_{j, k}}\right)_{\mu}=\left(\overrightarrow{\Delta_{j}^{2} \sigma_{j, k}}\right)_{\mu}+\left(\overrightarrow{\Delta_{k}^{2} \sigma_{j, k}}\right)_{\mu},
$$

where $\left(\overrightarrow{\Delta^{2} \sigma_{j, k}}\right)_{\mu},\left(\overrightarrow{\Delta_{j}^{2} \sigma_{j, k}}\right)_{\mu}$ and $\left(\overrightarrow{\Delta_{k}^{2} \sigma_{j, k}}\right)_{\mu}$ are respectively $\mu$-components of $\overrightarrow{\Delta^{2} \sigma_{j, k}}, \overrightarrow{\Delta_{j}^{2} \sigma_{j, k}}$ and $\overrightarrow{\Delta_{k}^{2} \sigma_{j, k}}$. Using the antisymmetric symbol shown in (3.80), (3.2) and (3.3) for $\mu=1,2,3$ and $j=1,2, \ldots, k$ and $k=1,2, \ldots, n$ are respectively written as

$$
\begin{aligned}
\left(\overrightarrow{\Delta_{j}^{2} \sigma_{j, k}}\right)_{\mu} & =\frac{1}{2} \varepsilon_{\alpha \beta \mu}\left(\overrightarrow{P_{j, k} P_{j-1, k}}\right)^{\alpha}\left(\overrightarrow{P_{j, k} P_{j-1, k-1}}\right)^{\beta}, \\
\left(\overrightarrow{\Delta_{k}^{2} \sigma_{j, k}}\right)_{\mu} & =\frac{1}{2} \varepsilon_{\alpha \beta \mu}\left(\overrightarrow{P_{j, k} P_{j-1, k-1}}\right)^{\alpha}\left(\overrightarrow{P_{j, k} P_{j, k-1}}\right)^{\beta},
\end{aligned}
$$

where the indices are summed over $\alpha, \beta=1,2,3$. Here, $\left(\overrightarrow{\Delta_{j}^{2} \sigma_{j, k}}\right)_{\mu}$ and $\left(\overrightarrow{\Delta_{k}^{2} \sigma_{j, k}}\right)_{\mu}$ for $\mu=1,2,3$ and $j=1,2, \ldots, k$ and $k=1,2, \ldots, n$ are respectively expressed as

$$
\begin{aligned}
\left(\overrightarrow{\Delta_{j}^{2} \sigma_{j, k}}\right)_{\mu} & =\frac{1}{2} \varepsilon_{\alpha \beta \mu} \Delta_{j}\left(x^{\alpha}\right)_{j, k} \Delta_{k}\left(x^{\beta}\right)_{j-1, k}, \\
\left(\overrightarrow{\Delta_{k}^{2} \sigma_{j, k}}\right)_{\mu} & =\frac{1}{2} \varepsilon_{\alpha \beta \mu} \Delta_{k}\left(x^{\beta}\right)_{j, k} \Delta_{j}\left(x^{\alpha}\right)_{j, k-1},
\end{aligned}
$$

where the indices are summed over $\alpha, \beta=1,2,3$. Substituting (3.84) and (3.85) into (3.81) for $\mu=1,2,3$ and $j=1,2, \ldots, k$ and $k=1,2, \ldots, n$, we may modify it as

$$
\left(\Delta^{2} \sigma_{\mu}\right)_{j, k}=\frac{1}{2} \varepsilon_{\alpha \beta \mu}\left[\Delta_{j}\left(x^{\alpha}\right)_{j, k} \Delta_{k}\left(x^{\beta}\right)_{j-1, k}+\Delta_{k}\left(x^{\beta}\right)_{j, k} \Delta_{j}\left(x^{\alpha}\right)_{j, k-1}\right],
$$

where the indices are summed over $\alpha, \beta=1,2,3$.

2. The lower-left finite area element vector $\left(\Delta^{2} \sigma_{\mu}\right)_{k, j} \equiv\left(\overrightarrow{\Delta^{2} \sigma_{k, j}}\right)_{\mu}$ for $\mu=1,2,3$ and $j=$ $1,2, \ldots, k$ and $k=1,2, \ldots, n$ is also disintegrated into

$$
\left(\overrightarrow{\Delta^{2} \sigma_{k, j}}\right)_{\mu}=\left(\overrightarrow{\Delta_{k}^{2} \sigma_{k, j}}\right)_{\mu}+\left(\overrightarrow{\Delta_{j}^{2} \sigma_{k, j}}\right)_{\mu}
$$

where $\left(\overrightarrow{\Delta^{2} \sigma_{k, j}}\right)_{\mu},\left(\overrightarrow{\Delta_{k}^{2} \sigma_{k, j}}\right)_{\mu}$ and $\left(\overrightarrow{\Delta_{j}^{2} \sigma_{k, j}}\right)_{\mu}$ are respectively $\mu$-components of $\overrightarrow{\Delta^{2} \sigma_{k, j}}, \overrightarrow{\Delta_{k}^{2} \sigma_{k, j}}$ and $\overrightarrow{\Delta_{j}^{2} \sigma_{k, j}}$. Using the antisymmetric symbol shown in (3.80), (3.5) and (3.6) for $\mu=1,2,3$ 
and $j=1,2, \ldots, k$ and $k=1,2, \ldots, n$ are respectively written as

$$
\begin{aligned}
& \left(\overrightarrow{\Delta_{k}^{2} \sigma_{k, j}}\right)_{\mu}=\frac{1}{2} \varepsilon_{\alpha \beta \mu}\left(\overrightarrow{P_{k, j} P_{k-1, j}}\right)^{\alpha}\left(\overrightarrow{P_{k, j} P_{k-1, j-1}}\right)^{\beta}, \\
& \left(\overrightarrow{\Delta_{j}^{2} \sigma_{k, j}}\right)_{\mu}=\frac{1}{2} \varepsilon_{\alpha \beta \mu}\left(\overrightarrow{P_{k, j} P_{k-1, j-1}}\right)^{\alpha}\left(\overrightarrow{P_{k, j} P_{k, j-1}}\right)^{\beta},
\end{aligned}
$$

where the indices are summed over $\alpha, \beta=1,2,3$. Here, $\left(\overrightarrow{\Delta_{k}^{2} \sigma_{k, j}}\right)_{\mu}$ and $\left(\overrightarrow{\Delta_{j}^{2} \sigma_{k, j}}\right)_{\mu}$ for $\mu=1,2,3$ and $j=1,2, \ldots, k$ and $k=1,2, \ldots, n$ are respectively expressed as

$$
\begin{aligned}
& \left(\overrightarrow{\Delta_{k}^{2} \sigma_{k, j}}\right)_{\mu}=\frac{1}{2} \varepsilon_{\alpha \beta \mu} \Delta_{k}\left(x^{\alpha}\right)_{k, j} \Delta_{j}\left(x^{\beta}\right)_{k-1, j}, \\
& \left(\overrightarrow{\Delta_{j}^{2} \sigma_{k, j}}\right)_{\mu}=\frac{1}{2} \varepsilon_{\alpha \beta \mu} \Delta_{j}\left(x^{\beta}\right)_{k, j} \Delta_{k}\left(x^{\alpha}\right)_{k, j-1},
\end{aligned}
$$

where the indices are summed over $\alpha, \beta=1,2,3$. Substituting (3.90) and (3.91) into (3.87) for $\mu=1,2,3$ and $j=1,2, \ldots, k$ and $k=1,2, \ldots, n$, we may modify it as

$$
\left(\Delta^{2} \sigma_{\mu}\right)_{k, j}=\frac{1}{2} \varepsilon_{\alpha \beta \mu}\left[\Delta_{k}\left(x^{\alpha}\right)_{k, j} \Delta_{j}\left(x^{\beta}\right)_{k-1, j}+\Delta_{j}\left(x^{\beta}\right)_{k, j} \Delta_{k}\left(x^{\alpha}\right)_{k, j-1}\right]
$$

where the indices are summed over $\alpha, \beta=1,2,3$

\subsection{The divergence theorem of a triangular integral in the 3D space}

In the following subsection, we prepare definitions and notations for extending Theorem 2 and Corollary 2 in the 3D space. Assume there are three functions $X=X(x, y, z)$, $Y=Y(x, y, z)$ and $Z=Z(x, y, z)$ of the three variables $x, y$ and $z$ in the Cartesian coordinates of the 3D space. Suppose there are two sets of double dependent sequences (3.93) and (3.94) as follows. Denoting $X^{1}=X, X^{2}=Y$ and $X^{3}=Z$, the two sets of integrand vectors $\overrightarrow{W_{j, k}}$ and $\overrightarrow{W_{k, j}}$ for $\mu=1,2,3$ and $j=0,1,2, \ldots, k$ and $k=0,1,2, \ldots, n$ are expressed as

$$
\begin{aligned}
& \overrightarrow{W_{j, k}} \equiv\left(X^{\mu}\right)_{j, k}=\left(X_{j, k}, Y_{j, k}, Z_{j, k}\right), \\
& \overrightarrow{W_{k, j}} \equiv\left(X^{\mu}\right)_{k, j}=\left(X_{k, j}, Y_{k, j}, Z_{k, j}\right) .
\end{aligned}
$$

For integrand vectors $\overrightarrow{W_{j, k}}=\left(X_{j, k}, Y_{j, k}, Z_{j, k}\right)$ and $\overrightarrow{W_{k, j}}=\left(X_{k, j}, Y_{k, j}, Z_{k, j}\right)$, we introduce the following double dependent sums:

$$
\begin{aligned}
& \sum_{k=1}^{n} \sum_{j=1}^{k}\left(\overrightarrow{W_{j, k}} \cdot \overrightarrow{\Delta^{2} \sigma_{j, k}}+\overrightarrow{W_{k, j}} \cdot \overrightarrow{\Delta^{2} \sigma_{k, j}}\right) \\
& \quad \equiv \sum_{k=1}^{n} \sum_{j=1}^{k}\left[\left(X^{\mu}\right)_{j, k}\left(\Delta^{2} \sigma_{\mu}\right)_{j, k}+\left(X^{\mu}\right)_{k, j}\left(\Delta^{2} \sigma_{\mu}\right)_{k, j}\right]
\end{aligned}
$$

where the index is summed over $\mu=1,2,3$. In order to prove Theorem $3,\left(X^{\mu}\right)_{j, k}$ and $\left(X^{\mu}\right)_{k, j}$ are respectively modified in Lemmata 4 and 5 .

1. The total increments of $\left(X^{\mu}\right)_{i, 0}$ for $\mu=1,2,3$ and $i=1,2, \ldots, j$ are denoted as

$$
\Delta_{i}\left(X^{\mu}\right)_{i, 0} \equiv\left(X^{\mu}\right)_{i, 0}-\left(X^{\mu}\right)_{i-1,0}
$$




$$
=X^{\mu}\left(x_{i, 0}, y_{i, 0}, z_{i, 0}\right)-X^{\mu}\left(x_{i-1,0}, y_{i-1,0}, z_{i-1,0}\right)
$$

The increments of $\left(x^{\gamma}\right)_{i, 0}$ for $\gamma=1,2,3$ and $i=1,2, \ldots, j$ are denoted as

$$
\Delta_{i}\left(x^{\gamma}\right)_{i, 0} \equiv\left(x^{\gamma}\right)_{i, 0}-\left(x^{\gamma}\right)_{i-1,0} .
$$

The partial increments of $\left(X^{\mu}\right)_{i, 0}$ for $\mu=1,2,3$ and $i=1,2, \ldots, j$ are denoted as

$$
\begin{aligned}
& \Delta X^{\mu}\left[\Delta_{i} x_{i, 0}\right] \equiv X^{\mu}\left(x_{i, 0}, y_{i, 0}, z_{i, 0}\right)-X^{\mu}\left(x_{i-1,0}, y_{i, 0}, z_{i, 0}\right) \\
& \Delta X^{\mu}\left[\Delta_{i} y_{i, 0}\right] \equiv X^{\mu}\left(x_{i-1,0}, y_{i, 0}, z_{i, 0}\right)-X^{\mu}\left(x_{i-1,0}, y_{i-1,0}, z_{i, 0}\right), \\
& \Delta X^{\mu}\left[\Delta_{i} z_{i, 0}\right] \equiv X^{\mu}\left(x_{i-1,0}, y_{i-1,0}, z_{i, 0}\right)-X^{\mu}\left(x_{i-1,0}, y_{i-1,0}, z_{i-1,0}\right) .
\end{aligned}
$$

2. The total increments of $\left(X^{\mu}\right)_{j, h}$ for $\mu=1,2,3$ and $j, h=1,2, \ldots, k$ are denoted as

$$
\begin{aligned}
\Delta_{h}\left(X^{\mu}\right)_{j, h} & \equiv\left(X^{\mu}\right)_{j, h}-\left(X^{\mu}\right)_{j, h-1} \\
& =X^{\mu}\left(x_{j, h}, y_{j, h}, z_{j, h}\right)-X^{\mu}\left(x_{j, h-1}, y_{j, h-1}, z_{j, h-1}\right) .
\end{aligned}
$$

The increments of $\left(x^{\gamma}\right)_{j, h}$ for $\gamma=1,2,3$ and $j, h=1,2, \ldots, k$ are denoted as

$$
\Delta_{h}\left(x^{\gamma}\right)_{j, h} \equiv\left(x^{\gamma}\right)_{j, h}-\left(x^{\gamma}\right)_{j, h-1} .
$$

The partial increments of $\left(X^{\mu}\right)_{j, h}$ for $\mu=1,2,3$ and $j, h=1,2, \ldots, k$ are denoted as

$$
\begin{aligned}
& \Delta X^{\mu}\left[\Delta_{h} x_{j, h}\right] \equiv X^{\mu}\left(x_{j, h}, y_{j, h}, z_{j, h}\right)-X^{\mu}\left(x_{j, h-1}, y_{j, h}, z_{j, h}\right), \\
& \Delta X^{\mu}\left[\Delta_{h} y_{j, h}\right] \equiv X^{\mu}\left(x_{j, h-1}, y_{j, h}, z_{j, h}\right)-X^{\mu}\left(x_{j, h-1}, y_{j, h-1}, z_{j, h}\right), \\
& \Delta X^{\mu}\left[\Delta_{h} z_{j, h}\right] \equiv X^{\mu}\left(x_{j, h-1}, y_{j, h-1}, z_{j, h}\right)-X^{\mu}\left(x_{j, h-1}, y_{j, h-1}, z_{j, h-1}\right) .
\end{aligned}
$$

Lemma 4 In the case of $(j, k)$ for $\mu=1,2,3$ and $j=1,2, \ldots, k$ and $k=1,2, \ldots, n$, the following holds:

$$
\left(X^{\mu}\right)_{j, k}=\left(X^{\mu}\right)_{0,0}+\sum_{i=1}^{j} \frac{\Delta X^{\mu}\left[\Delta_{i}\left(x^{\gamma}\right)_{i, 0}\right]}{\Delta_{i}\left(x^{\gamma}\right)_{i, 0}} \Delta_{i}\left(x^{\gamma}\right)_{i, 0}+\sum_{h=1}^{k} \frac{\Delta X^{\mu}\left[\Delta_{h}\left(x^{\gamma}\right)_{j, h}\right]}{\Delta_{h}\left(x^{\gamma}\right)_{j, h}} \Delta_{h}\left(x^{\gamma}\right)_{j, h}
$$

where the index is summed over $\gamma=1,2,3$.

Proof Using (3.96) and (3.101), $\left(X^{\mu}\right)_{j, k}$ for $\mu=1,2,3$ and $j=1,2, \ldots, k$ and $k=1,2, \ldots, n$ is disintegrated into

$$
\left(X^{\mu}\right)_{j, k}=\left(X^{\mu}\right)_{0,0}+\sum_{i=1}^{j} \Delta_{i}\left(X^{\mu}\right)_{i, 0}+\sum_{h=1}^{k} \Delta_{h}\left(X^{\mu}\right)_{j, h}
$$

1. Substituting (3.98), (3.99) and (3.100) into (3.96) for $\mu=1,2,3$ and $i=1,2, \ldots, j$, we obtain

$$
\Delta_{i}\left(X^{\mu}\right)_{i, 0}=\Delta X^{\mu}\left[\Delta_{i} x_{i, 0}\right]+\Delta X^{\mu}\left[\Delta_{i} y_{i, 0}\right]+\Delta X^{\mu}\left[\Delta_{i} z_{i, 0}\right]
$$




$$
=\frac{\Delta X^{\mu}\left[\Delta_{i}\left(x^{\gamma}\right)_{i, 0}\right]}{\Delta_{i}\left(x^{\gamma}\right)_{i, 0}} \Delta_{i}\left(x^{\gamma}\right)_{i, 0}
$$

where the index is summed over $\gamma=1,2,3$.

2. Substituting (3.103), (3.104) and (3.105) into (3.101) for $\mu=1,2,3$ and $j, h=1,2, \ldots, k$, we obtain

$$
\begin{aligned}
\Delta_{h}\left(X^{\mu}\right)_{j, h} & =\Delta X^{\mu}\left[\Delta_{h} x_{j, h}\right]+\Delta X^{\mu}\left[\Delta_{h} y_{j, h}\right]+\Delta X^{\mu}\left[\Delta_{h} z_{j, h}\right] \\
& =\frac{\Delta X^{\mu}\left[\Delta_{h}\left(x^{\gamma}\right)_{j, h}\right]}{\Delta_{h}\left(x^{\gamma}\right)_{j, h}} \Delta_{h}\left(x^{\gamma}\right)_{j, h^{\prime}}
\end{aligned}
$$

where the index is summed over $\gamma=1,2,3$.

Substituting (3.108) and (3.109) into (3.107), we obtain (3.106).

Q.E.D.

Lemma 5 In the case of $(k, j)$ for $\mu=1,2,3$ and $j=1,2, \ldots, k$ and $k=1,2, \ldots, n$, the following holds:

$$
\begin{aligned}
\left(X^{\mu}\right)_{k, j}= & \left(X^{\mu}\right)_{0,0}+\sum_{i=1}^{j} \frac{\Delta_{i}\left(X^{\mu}\right)\left[\Delta_{i}\left(x^{\gamma}\right)_{0, i}\right]}{\Delta_{i}\left(x^{\gamma}\right)_{0, i}} \Delta_{i}\left(x^{\gamma}\right)_{0, i} \\
& +\sum_{h=1}^{k} \frac{\Delta_{h}\left(X^{\mu}\right)\left[\Delta_{h}\left(x^{\gamma}\right)_{h, j}\right]}{\Delta_{h}\left(x^{\gamma}\right)_{h, j}} \Delta_{h}\left(x^{\gamma}\right)_{h, j},
\end{aligned}
$$

where the index is summed over $\gamma=1,2,3$.

Proof In the similar manner as Lemma 4, we obtain (3.110).

Q.E.D.

Our triangular double and triple integrals and the divergence theorem of a triangular integral in the 3D space are shown as follows, where $D$ is a domain and $\partial D$ is the boundary of the domain $D$ in the $3 \mathrm{D}$ space.

Definition 5 A triangular area double integral in the 3D space $\iint_{\partial D} X^{\mu} d^{2} \sigma_{\mu}$ is defined as

$$
\iint_{\partial D} X^{\mu} d^{2} \sigma_{\mu}=\lim _{n \rightarrow \infty} \sum_{k=1}^{n} \sum_{j=1}^{k}\left[\left(X^{\mu}\right)_{j, k}\left(\Delta^{2} \sigma_{\mu}\right)_{j, k}+\left(X^{\mu}\right)_{k, j}\left(\Delta^{2} \sigma_{\mu}\right)_{k, j}\right]
$$

where the index is summed over $\mu=1,2,3$.

Definition 6 A triangular triple integral, integrands of which are partial differentials in the $3 \mathrm{D}$ space $\iiint_{D} \frac{\partial X^{\prime \prime \mu}}{\partial x^{\prime \prime \gamma}} d x^{\prime \prime \gamma} d^{2} \sigma_{\mu}$, is defined as

$$
\begin{aligned}
\iiint_{D} \frac{\partial X^{\prime \prime \mu}}{\partial x^{\prime \prime \gamma}} d x^{\prime \prime \gamma} d^{2} \sigma_{\mu}= & \lim _{n \rightarrow \infty} \sum_{k=1}^{n} \sum_{j=1}^{k}\left[\sum_{i=1}^{j}\left(\frac{\Delta_{i}\left(X^{\mu}\right)\left[\Delta_{i}\left(x^{\gamma}\right)_{i, 0}\right]}{\Delta_{i}\left(x^{\gamma}\right)_{i, 0}} \Delta_{i}\left(x^{\gamma}\right)_{i, 0}\right)\right. \\
& \left.+\sum_{h=1}^{k}\left(\frac{\Delta_{h}\left(X^{\mu}\right)\left[\Delta_{h}\left(x^{\gamma}\right)_{j, h}\right]}{\Delta_{h}\left(x^{\gamma}\right)_{j, h}} \Delta_{h}\left(x^{\gamma}\right)_{j, h}\right)\right]\left(\Delta^{2} \sigma_{\mu}\right)_{j, k} \\
& +\lim _{n \rightarrow \infty} \sum_{k=1}^{n} \sum_{j=1}^{k}\left[\sum_{i=1}^{j}\left(\frac{\Delta_{i}\left(X^{\mu}\right)\left[\Delta_{i}\left(x^{\gamma}\right)_{0, i}\right]}{\Delta_{i}\left(x^{\gamma}\right)_{0, i}} \Delta_{i}\left(x^{\gamma}\right)_{0, i}\right)\right.
\end{aligned}
$$




$$
\left.+\sum_{h=1}^{k}\left(\frac{\Delta_{h}\left(X^{\mu}\right)\left[\Delta_{h}\left(x^{\gamma}\right)_{h, j}\right]}{\Delta_{h}\left(x^{\gamma}\right)_{h, j}} \Delta_{h}\left(x^{\gamma}\right)_{h, j}\right)\right]\left(\Delta^{2} \sigma_{\mu}\right)_{k, j}
$$

where the indices are summed over $\gamma, \mu=1,2,3$.

The following proposition is necessary for the condition (3.118) of Theorem 3.

Proposition 3 Denote constants as $C^{1}=C^{x}, C^{2}=C^{y}$ and $C^{3}=C^{z}$, then

$$
C^{\mu} \iint_{\partial D} d^{2} \sigma_{\mu}=C^{\mu} \lim _{n \rightarrow \infty} \sum_{k=1}^{n} \sum_{j=1}^{k}\left[\left(\Delta^{2} \sigma_{\mu}\right)_{j, k}+\left(\Delta^{2} \sigma_{\mu}\right)_{k, j}\right]
$$

holds, where the index is summed over $\mu=1,2,3$.

Proof In the case of $X^{1}=1, X^{2}=0$ and $X^{3}=0$, Definition 5 is reduced to be

$$
\iint_{\partial D} d^{2} \sigma_{x}=\lim _{n \rightarrow \infty} \sum_{k=1}^{n} \sum_{j=1}^{k}\left[\left(\Delta^{2} \sigma_{x}\right)_{j, k}+\left(\Delta^{2} \sigma_{x}\right)_{k, j}\right] .
$$

In the case of $X^{1}=0, X^{2}=1$ and $X^{3}=0$, Definition 5 is reduced to be

$$
\iint_{\partial D} d^{2} \sigma_{y}=\lim _{n \rightarrow \infty} \sum_{k=1}^{n} \sum_{j=1}^{k}\left[\left(\Delta^{2} \sigma_{y}\right)_{j, k}+\left(\Delta^{2} \sigma_{y}\right)_{k, j}\right] .
$$

In the case of $X^{1}=0, X^{2}=0$ and $X^{3}=1$, Definition 5 is reduced to be

$$
\iint_{\partial D} d^{2} \sigma_{z}=\lim _{n \rightarrow \infty} \sum_{k=1}^{n} \sum_{j=1}^{k}\left[\left(\Delta^{2} \sigma_{z}\right)_{j, k}+\left(\Delta^{2} \sigma_{z}\right)_{k, j}\right] .
$$

A linear combination of (3.114), (3.115) and (3.116) is

$$
\begin{aligned}
& C^{x} \iint_{\partial D} d^{2} \sigma_{x}+C^{y} \iint_{\partial D} d^{2} \sigma_{y}+C^{z} \iint_{\partial D} d^{2} \sigma_{z} \\
& =C^{x} \lim _{n \rightarrow \infty} \sum_{k=1}^{n} \sum_{j=1}^{k}\left[\left(\Delta^{2} \sigma_{x}\right)_{j, k}+\left(\Delta^{2} \sigma_{x}\right)_{k, j}\right]+C^{y} \lim _{n \rightarrow \infty} \sum_{k=1}^{n} \sum_{j=1}^{k}\left[\left(\Delta^{2} \sigma_{y}\right)_{j, k}+\left(\Delta^{2} \sigma_{y}\right)_{k, j}\right] \\
& \quad+C^{z} \lim _{n \rightarrow \infty} \sum_{k=1}^{n} \sum_{j=1}^{k}\left[\left(\Delta^{2} \sigma_{z}\right)_{j, k}+\left(\Delta^{2} \sigma_{z}\right)_{k, j}\right] .
\end{aligned}
$$

Theorem 3 shown below is the 3D version of Theorem 2, since it derives (3.123) in Corollary 3. The condition (3.118) or (3.121) is the 3D version of the condition (2.17) or (2.39).

Theorem 3 (The divergence theorem of a triangular integral in the 3D space) Assume D is a domain and $\partial D$ is the boundary of the domain in the xyz-space, expressed in the Cartesian coordinates $(x, y, z) \in \mathbb{R}^{3}$. Let $X^{1}=X=X(x, y, z), X^{2}=Y=Y(x, y, z)$ and $X^{3}=Z=Z(x, y, z)$ 
be partially differentiable functions with respect to $x^{1}=x, x^{2}=y$ and $x^{3}=z$ in $D$. In the case of a closed $2 D$ surface which satisfies

$$
C^{\mu} \iint_{\partial D} d^{2} \sigma_{\mu}=0
$$

the divergence theorem of a triangular integral in the $3 D$ space holds:

$$
\oiint_{\partial D} X^{\mu} d^{2} \sigma_{\mu}=\iiint_{D} \frac{\partial X^{\prime \prime \mu}}{\partial x^{\prime \prime \gamma}} d x^{\prime \prime \gamma} d^{2} \sigma_{\mu}
$$

where the indices are summed over $\gamma, \mu=1,2,3$ and $C^{\mu}$ are constants.

Proof Substituting (3.106) and (3.110) into the right-hand side of (3.95), we obtain

$$
\begin{aligned}
& \sum_{k=1}^{n} \sum_{j=1}^{k}\left[\left(X^{\mu}\right)_{j, k}\left(\Delta^{2} \sigma_{\mu}\right)_{j, k}+\left(X^{\mu}\right)_{k, j}\left(\Delta^{2} \sigma_{\mu}\right)_{k, j}\right] \\
& =\sum_{k=1}^{n} \sum_{j=1}^{k}\left[\sum_{i=1}^{j}\left(\frac{\Delta_{i}\left(X^{\mu}\right)\left[\Delta_{i}\left(x^{\gamma}\right)_{i, 0}\right]}{\Delta_{i}\left(x^{\gamma}\right)_{i, 0}} \Delta_{i}\left(x^{\gamma}\right)_{i, 0}\right)\right. \\
& \left.\quad+\sum_{h=1}^{k}\left(\frac{\Delta_{h}\left(X^{\mu}\right)\left[\Delta_{h}\left(x^{\gamma}\right)_{j, h}\right]}{\Delta_{h}\left(x^{\gamma}\right)_{j, h}} \Delta_{h}\left(x^{\gamma}\right)_{j, h}\right)\right]\left(\Delta^{2} \sigma_{\mu}\right)_{j, k} \\
& \quad+\sum_{k=1}^{n} \sum_{j=1}^{k}\left[\sum_{i=1}^{j}\left(\frac{\Delta_{i}\left(X^{\mu}\right)\left[\Delta_{i}\left(x^{\gamma}\right)_{0, i}\right]}{\Delta_{i}\left(x^{\gamma}\right)_{0, i}} \Delta_{i}\left(x^{\gamma}\right)_{0, i}\right)\right. \\
& \left.\quad+\sum_{h=1}^{k}\left(\frac{\Delta_{h}\left(X^{\mu}\right)\left[\Delta_{h}\left(x^{\gamma}\right)_{h, j}\right]}{\Delta_{h}\left(x^{\gamma}\right)_{h, j}} \Delta_{h}\left(x^{\gamma}\right)_{h, j}\right)\right]\left(\Delta^{2} \sigma_{\mu}\right)_{k, j} \\
& \quad+\left(X^{\mu}\right)_{0,0} \sum_{k=1}^{n} \sum_{j=1}^{k}\left[\left(\Delta^{2} \sigma_{\mu}\right)_{j, k}+\left(\Delta^{2} \sigma_{\mu}\right)_{k, j}\right],
\end{aligned}
$$

where the indices are summed over $\gamma, \mu=1,2,3$. Using Proposition 3, (3.118) is rewritten as

$$
\left(X^{\mu}\right)_{0,0} \lim _{n \rightarrow \infty} \sum_{k=1}^{n} \sum_{j=1}^{k}\left[\left(\Delta^{2} \sigma_{\mu}\right)_{j, k}+\left(\Delta^{2} \sigma_{\mu}\right)_{k, j}\right]=0,
$$

where the index is summed over $\mu=1,2,3$. The limit at infinity $n \rightarrow \infty$ of (3.120) under the condition of a closed surface (3.121) is expressed as (3.119) by Definitions 5 and 6 .

Q.E.D.

Remark The divergence theorem (3.119) shown above is not the conventional one (1.1), which has been familiar to us for two hundred years. The inner product of the nabla and a vector (1.4) is not explicitly shown as an integrand in (3.119).

Corollary 3 shown below derived from Theorem 3 is the 3D version of Corollary 2 derived from Theorem 2. Infinitesimal volume element $d^{3} V$ is introduced as $d^{3} V=$ $\frac{1}{3} d x^{\prime \prime \gamma} d^{2} \sigma_{\gamma}$, where the index is summed over $\gamma=1,2,3$. 
Corollary 3 (Derivation of the inner product of the nabla and a vector) In the case of

$$
\oiint_{\partial D} X^{\mu} d^{2} \sigma_{\mu}=\iiint_{D} \rho d^{3} V
$$

where $\rho=\rho(x, y, z)$ is a given function and the index is summed over $\mu=1,2,3$, the following holds:

$$
\frac{\partial X^{\mu}}{\partial x^{\mu}}=\rho
$$

Proof Applying Theorem 3 to the left-hand side of (3.122), it is modified to be

$$
\begin{aligned}
& \lim _{n \rightarrow \infty} \sum_{k=1}^{n} \sum_{j=1}^{k} \sum_{i=1}^{j} \frac{\Delta_{i}\left(X^{\mu}\right)\left[\Delta_{i}\left(x^{\gamma}\right)_{i, 0}\right]}{\Delta_{i}\left(x^{\gamma}\right)_{i, 0}} \frac{1}{2} \varepsilon_{\alpha \beta \mu} \Delta_{i}\left(x^{\gamma}\right)_{i, 0} \\
& \times\left[\Delta_{j}\left(x^{\alpha}\right)_{j, k} \Delta_{k}\left(x^{\beta}\right)_{j-1, k}+\Delta_{k}\left(x^{\beta}\right)_{j, k} \Delta_{j}\left(x^{\alpha}\right)_{j, k-1}\right] \\
& +\lim _{n \rightarrow \infty} \sum_{k=1}^{n} \sum_{j=1}^{k} \sum_{h=1}^{k} \frac{\Delta_{h}\left(X^{\mu}\right)\left[\Delta_{h}\left(x^{\gamma}\right)_{j, h}\right]}{\Delta_{h}\left(x^{\gamma}\right)_{j, h}} \frac{1}{2} \varepsilon_{\alpha \beta \mu} \Delta_{h}\left(x^{\gamma}\right)_{j, h} \\
& \times\left[\Delta_{j}\left(x^{\alpha}\right)_{j, k} \Delta_{k}\left(x^{\beta}\right)_{j-1, k}+\Delta_{k}\left(x^{\beta}\right)_{j, k} \Delta_{j}\left(x^{\alpha}\right)_{j, k-1}\right] \\
& +\lim _{n \rightarrow \infty} \sum_{k=1}^{n} \sum_{j=1}^{k} \sum_{i=1}^{j} \frac{\Delta_{i}\left(X^{\mu}\right)\left[\Delta_{i}\left(x^{\gamma}\right)_{0, i}\right]}{\Delta_{i}\left(x^{\gamma}\right)_{0, i}} \frac{1}{2} \varepsilon_{\alpha \beta \mu} \Delta_{i}\left(x^{\gamma}\right)_{0, i} \\
& \times\left[\Delta_{k}\left(x^{\alpha}\right)_{k, j} \Delta_{j}\left(x^{\beta}\right)_{k-1, j}+\Delta_{j}\left(x^{\beta}\right)_{k, j} \Delta_{k}\left(x^{\alpha}\right)_{k, j-1}\right] \\
& +\lim _{n \rightarrow \infty} \sum_{k=1}^{n} \sum_{j=1}^{k} \sum_{h=1}^{k} \frac{\Delta_{h}\left(X^{\mu}\right)\left[\Delta_{h}\left(x^{\gamma}\right)_{h, j}\right]}{\Delta_{h}\left(x^{\gamma}\right)_{h, j}} \frac{1}{2} \varepsilon_{\alpha \beta \mu} \Delta_{h}\left(x^{\gamma}\right)_{h, j} \\
& \times\left[\Delta_{k}\left(x^{\alpha}\right)_{k, j} \Delta_{j}\left(x^{\beta}\right)_{k-1, j}+\Delta_{j}\left(x^{\beta}\right)_{k, j} \Delta_{k}\left(x^{\alpha}\right)_{k, j-1}\right] \\
& =\lim _{n \rightarrow \infty} \sum_{k=1}^{n} \sum_{j=1}^{k} \sum_{i=1}^{j} \frac{\rho}{3 !} \varepsilon_{\alpha \beta \gamma} \Delta_{i}\left(x^{\gamma}\right)_{i, 0}\left[\Delta_{j}\left(x^{\alpha}\right)_{j, k} \Delta_{k}\left(x^{\beta}\right)_{j-1, k}+\Delta_{k}\left(x^{\beta}\right)_{j, k} \Delta_{j}\left(x^{\alpha}\right)_{j, k-1}\right] \\
& +\lim _{n \rightarrow \infty} \sum_{k=1}^{n} \sum_{j=1}^{k} \sum_{h=1}^{k} \frac{\rho}{3 !} \varepsilon_{\alpha \beta \gamma} \Delta_{h}\left(x^{\gamma}\right)_{j, h}\left[\Delta_{j}\left(x^{\alpha}\right)_{j, k} \Delta_{k}\left(x^{\beta}\right)_{j-1, k}+\Delta_{k}\left(x^{\beta}\right)_{j, k} \Delta_{j}\left(x^{\alpha}\right)_{j, k-1}\right] \\
& +\lim _{n \rightarrow \infty} \sum_{k=1}^{n} \sum_{j=1}^{k} \sum_{i=1}^{j} \frac{\rho}{3 !} \varepsilon_{\alpha \beta \gamma} \Delta_{i}\left(x^{\gamma}\right)_{0, i}\left[\Delta_{k}\left(x^{\alpha}\right)_{k, j} \Delta_{j}\left(x^{\beta}\right)_{k-1, j}+\Delta_{j}\left(x^{\beta}\right)_{k, j} \Delta_{k}\left(x^{\alpha}\right)_{k, j-1}\right] \\
& +\lim _{n \rightarrow \infty} \sum_{k=1}^{n} \sum_{j=1}^{k} \sum_{h=1}^{k} \frac{\rho}{3 !} \varepsilon_{\alpha \beta \gamma} \Delta_{h}\left(x^{\gamma}\right)_{h, j} \\
& \times\left[\Delta_{k}\left(x^{\alpha}\right)_{k, j} \Delta_{j}\left(x^{\beta}\right)_{k-1, j}+\Delta_{j}\left(x^{\beta}\right)_{k, j} \Delta_{k}\left(x^{\alpha}\right)_{k, j-1}\right],
\end{aligned}
$$

where the indices are summed over $\alpha, \beta, \gamma, \mu=1,2,3$. In order to hold (3.124) for any value of integral variables, the following four kinds of formulae in two categories are demanded.

1 . In the case of $i=1,2, \ldots, j$,

$$
\lim _{\Delta_{i}\left(x^{\gamma}\right)_{i, 0} \rightarrow 0} \frac{\Delta_{i}\left(X^{\mu}\right)\left[\Delta_{i}\left(x^{\gamma}\right)_{i, 0}\right]}{\Delta_{i}\left(x^{\gamma}\right)_{i, 0}} \frac{1}{2} \varepsilon_{\alpha \beta \mu}=\frac{\rho}{3 !} \varepsilon_{\alpha \beta \gamma}
$$




$$
\lim _{\Delta_{i}\left(x^{\gamma}\right)_{0, i} \rightarrow 0} \frac{\Delta_{i}\left(X^{\mu}\right)\left[\Delta_{i}\left(x^{\gamma}\right)_{0, i}\right]}{\Delta_{i}\left(x^{\gamma}\right)_{0, i}} \frac{1}{2} \varepsilon_{\alpha \beta \mu}=\frac{\rho}{3 !} \varepsilon_{\alpha \beta \gamma}
$$

for $\alpha, \beta, \gamma=1,2,3$, where the index is summed over $\mu=1,2,3$.

2. In the case of $h, j=1,2, \ldots, k$,

$$
\begin{aligned}
\lim _{\Delta_{h}\left(x^{\gamma}\right)_{j, h \rightarrow 0} \rightarrow 0} \frac{\Delta_{h}\left(X^{\mu}\right)\left[\Delta_{h}\left(x^{\gamma}\right)_{j, h}\right]}{\Delta_{h}\left(x^{\gamma}\right)_{j, h}} \frac{1}{2} \varepsilon_{\alpha \beta \mu} & =\frac{\rho}{3 !} \varepsilon_{\alpha \beta \gamma}, \\
\lim _{\Delta_{h}\left(x^{\gamma}\right)_{h, j} \rightarrow 0} \frac{\Delta_{h}\left(X^{\mu}\right)\left[\Delta_{h}\left(x^{\gamma}\right)_{h, j}\right]}{\Delta_{h}\left(x^{\gamma}\right)_{h, j}} \frac{1}{2} \varepsilon_{\alpha \beta \mu} & =\frac{\rho}{3 !} \varepsilon_{\alpha \beta \gamma}
\end{aligned}
$$

for $\alpha, \beta, \gamma=1,2,3$, where the index is summed over $\mu=1,2,3$.

Multiplying $\varepsilon^{\alpha \beta \gamma}$ to the both sides of the four kinds of formulae (3.125), (3.126), (3.127) and (3.128), they are reduced to be the differential equation (3.123).

Remark We finally obtained the inner product of the nabla and a vector (1.4) by using the antisymmetric symbol, which has explicitly appeared in the conventional divergence theorem (1.1) as the integrand.

Example 2 (A spherical example of the divergence theorem of a triangular integral in the 3D space) First of all, we see an example which satisfies the condition (3.121) of the divergence theorem (3.118) or (3.121). The condition (3.121) in Theorem 3 is expressed in component representation as

$$
\begin{aligned}
& X_{0,0} \lim _{n \rightarrow \infty} \sum_{k=1}^{n} \sum_{j=1}^{k}\left[\left(\Delta^{2} \sigma_{x}\right)_{j, k}+\left(\Delta^{2} \sigma_{x}\right)_{k, j}\right]+Y_{0,0} \lim _{n \rightarrow \infty} \sum_{k=1}^{n} \sum_{j=1}^{k}\left[\left(\Delta^{2} \sigma_{y}\right)_{j, k}+\left(\Delta^{2} \sigma_{y}\right)_{k, j}\right] \\
& +Z_{0,0} \lim _{n \rightarrow \infty} \sum_{k=1}^{n} \sum_{j=1}^{k}\left[\left(\Delta^{2} \sigma_{z}\right)_{j, k}+\left(\Delta^{2} \sigma_{z}\right)_{k, j}\right]=0 .
\end{aligned}
$$

We consider the case of $X^{\mu}=\frac{1}{3} x^{\mu}$. Using (A.1) and (A.2) or (A.14) and (A.15), we obtain $X_{0,0}=Y_{0,0}=0$. Thus, the condition (3.129) of the divergence theorem of a triangular integral is reduced to be

$$
Z_{0,0} \lim _{n \rightarrow \infty} \sum_{k=1}^{n} \sum_{j=1}^{k}\left[\left(\Delta^{2} \sigma_{z}\right)_{j, k}+\left(\Delta^{2} \sigma_{z}\right)_{k, j}\right]=0 .
$$

Using (A.3) or (A.16), we obtain $Z_{0,0}=\frac{a}{3}$. In the case of the sphere, (3.130) is satisfied. The details are shown in Appendix $\mathrm{C}$. It is concluded that we are able to apply Theorem 3 for this integrand $\vec{W}=\frac{b}{a} \vec{r}$ and the region of the sphere $x^{2}+y^{2}+z^{2}=\left(\frac{b}{a}\right)^{2}$.

Next, the left-hand side of (3.119) is calculated as follows. Calculations in detail are shown in Appendix B in the case of $b=\frac{1}{3} a$. The each value of the first term of the lefthand side of (3.120) is

$$
\lim _{n \rightarrow \infty} \sum_{k=1}^{n} \sum_{j=1}^{k} X_{j, k}\left(\Delta^{2} \sigma_{x}\right)_{j, k}=\frac{2}{9} \pi a^{3},
$$




$$
\begin{aligned}
& \lim _{n \rightarrow \infty} \sum_{k=1}^{n} \sum_{j=1}^{k} Y_{j, k}\left(\Delta^{2} \sigma_{y}\right)_{j, k}=\frac{2}{9} \pi a^{3}, \\
& \lim _{n \rightarrow \infty} \sum_{k=1}^{n} \sum_{j=1}^{k} Z_{j, k}\left(\Delta^{2} \sigma_{z}\right)_{j, k}=\frac{2}{9} \pi a^{3} .
\end{aligned}
$$

The each value of the second term of the left-hand side of (3.120) is

$$
\begin{aligned}
& \lim _{n \rightarrow \infty} \sum_{k=1}^{n} \sum_{j=1}^{k} X_{k, j}\left(\Delta^{2} \sigma_{x}\right)_{k, j}=\frac{2}{9} \pi a^{3}, \\
& \lim _{n \rightarrow \infty} \sum_{k=1}^{n} \sum_{j=1}^{k} Y_{k, j}\left(\Delta^{2} \sigma_{y}\right)_{k, j}=\frac{2}{9} \pi a^{3}, \\
& \lim _{n \rightarrow \infty} \sum_{k=1}^{n} \sum_{j=1}^{k} Z_{k, j}\left(\Delta^{2} \sigma_{z}\right)_{k, j}=\frac{2}{9} \pi a^{3} .
\end{aligned}
$$

Using (3.111) in Definition 5, the left-hand side of (3.119) is calculated to be

$$
\oiint_{\partial D} X^{\mu} d^{2} \sigma_{\mu}=\frac{4}{3} \pi a^{3}
$$

where the index is summed over $\mu=1,2,3$.

Finally, the right-hand side of (3.119) is calculated as follows. The first term of the righthand side of (3.120) is reduced to be

$$
\begin{aligned}
& \lim _{n \rightarrow \infty} \sum_{k=1}^{n} \sum_{j=1}^{k}\left[\sum_{i=1}^{j}\left(\frac{\Delta_{i}\left(X^{\mu}\right)\left[\Delta_{i}\left(x^{\gamma}\right)_{i, 0}\right]}{\Delta_{i}\left(x^{\gamma}\right)_{i, 0}} \Delta_{i}\left(x^{\gamma}\right)_{i, 0}\right)\right. \\
& \left.\quad+\sum_{h=1}^{k}\left(\frac{\Delta_{h}\left(X^{\mu}\right)\left[\Delta_{h}\left(x^{\gamma}\right)_{j, h}\right]}{\Delta_{h}\left(x^{\gamma}\right)_{j, h}} \Delta_{h}\left(x^{\gamma}\right)_{j, h}\right)\right]\left(\Delta^{2} \sigma_{\mu}\right)_{j, k} \\
& =\frac{1}{3} \lim _{n \rightarrow \infty} \sum_{k=1}^{n} \sum_{j=1}^{k}\left(x_{j, k}-x_{0,0}\right)\left(\Delta^{2} \sigma_{x}\right)_{j, k}+\frac{1}{3} \lim _{n \rightarrow \infty} \sum_{k=1}^{n} \sum_{j=1}^{k}\left(y_{j, k}-y_{0,0}\right)\left(\Delta^{2} \sigma_{y}\right)_{j, k} \\
& \quad+\frac{1}{3} \lim _{n \rightarrow \infty} \sum_{k=1}^{n} \sum_{j=1}^{k}\left(z_{j, k}-z_{0,0}\right)\left(\Delta^{2} \sigma_{z}\right)_{j, k}
\end{aligned}
$$

where the indices are summed over $\gamma, \mu=1,2,3$. The value of each term of the right-hand side of (3.138) is

$$
\begin{aligned}
& \frac{1}{3} \lim _{n \rightarrow \infty} \sum_{k=1}^{n} \sum_{j=1}^{k}\left(x_{j, k}-x_{0,0}\right)\left(\Delta^{2} \sigma_{x}\right)_{j, k}=\frac{2}{9} \pi a^{3}, \\
& \frac{1}{3} \lim _{n \rightarrow \infty} \sum_{k=1}^{n} \sum_{j=1}^{k}\left(y_{j, k}-y_{0,0}\right)\left(\Delta^{2} \sigma_{y}\right)_{j, k}=\frac{2}{9} \pi a^{3}, \\
& \frac{1}{3} \lim _{n \rightarrow \infty} \sum_{k=1}^{n} \sum_{j=1}^{k}\left(z_{j, k}-z_{0,0}\right)\left(\Delta^{2} \sigma_{z}\right)_{j, k}=\frac{1}{18} \pi a^{3} .
\end{aligned}
$$


Substituting (3.139), (3.140) and (3.141) into the right-hand side of (3.138), the value of the first term of the right-hand side of (3.120) is

$$
\begin{aligned}
& \lim _{n \rightarrow \infty} \sum_{k=1}^{n} \sum_{j=1}^{k}\left[\sum_{i=1}^{j}\left(\frac{\Delta_{i}\left(X^{\mu}\right)\left[\Delta_{i}\left(x^{\gamma}\right)_{i, 0}\right]}{\Delta_{i}\left(x^{\gamma}\right)_{i, 0}} \Delta_{i}\left(x^{\gamma}\right)_{i, 0}\right)\right. \\
& \left.\quad+\sum_{h=1}^{k}\left(\frac{\Delta_{h}\left(X^{\mu}\right)\left[\Delta_{h}\left(x^{\gamma}\right)_{j, h}\right]}{\Delta_{h}\left(x^{\gamma}\right)_{j, h}} \Delta_{h}\left(x^{\gamma}\right)_{j, h}\right)\right]\left(\Delta^{2} \sigma_{\mu}\right)_{j, k}=\frac{1}{2} \pi a^{3},
\end{aligned}
$$

where the indices are summed over $\gamma, \mu=1,2,3$. The second term of the right-hand side of (3.120) is reduced to be

$$
\begin{aligned}
& \lim _{n \rightarrow \infty} \sum_{k=1}^{n} \sum_{j=1}^{k}\left[\sum_{i=1}^{j}\left(\frac{\Delta_{i}\left(X^{\mu}\right)\left[\Delta_{i}\left(x^{\gamma}\right)_{0, i}\right]}{\Delta_{i}\left(x^{\gamma}\right)_{0, i}} \Delta_{i}\left(x^{\gamma}\right)_{0, i}\right)\right. \\
& \left.\quad+\sum_{h=1}^{k}\left(\frac{\Delta_{h}\left(X^{\mu}\right)\left[\Delta_{h}\left(x^{\gamma}\right)_{h, j}\right]}{\Delta_{h}\left(x^{\gamma}\right)_{h, j}} \Delta_{h}\left(x^{\gamma}\right)_{h, j}\right)\right]\left(\Delta^{2} \sigma_{\mu}\right)_{k, j} \\
& =\frac{1}{3} \lim _{n \rightarrow \infty} \sum_{k=1}^{n} \sum_{j=1}^{k}\left(x_{k, j}-x_{0,0}\right)\left(\Delta^{2} \sigma_{x}\right)_{k, j}+\frac{1}{3} \lim _{n \rightarrow \infty} \sum_{k=1}^{n} \sum_{j=1}^{k}\left(y_{k, j}-y_{0,0}\right)\left(\Delta^{2} \sigma_{y}\right)_{k, j} \\
& \quad+\frac{1}{3} \lim _{n \rightarrow \infty} \sum_{k=1}^{n} \sum_{j=1}^{k}\left(z_{k, j}-z_{0,0}\right)\left(\Delta^{2} \sigma_{z}\right)_{k, j},
\end{aligned}
$$

where the indices are summed over $\gamma, \mu=1,2,3$. The value of each term of the right-hand side of (3.143) is

$$
\begin{aligned}
& \frac{1}{3} \lim _{n \rightarrow \infty} \sum_{k=1}^{n} \sum_{j=1}^{k}\left(x_{k, j}-x_{0,0}\right)\left(\Delta^{2} \sigma_{x}\right)_{k, j}=\frac{2}{9} \pi a^{3}, \\
& \frac{1}{3} \lim _{n \rightarrow \infty} \sum_{k=1}^{n} \sum_{j=1}^{k}\left(y_{k, j}-y_{0,0}\right)\left(\Delta^{2} \sigma_{y}\right)_{k, j}=\frac{2}{9} \pi a^{3}, \\
& \frac{1}{3} \lim _{n \rightarrow \infty} \sum_{k=1}^{n} \sum_{j=1}^{k}\left(z_{k, j}-z_{0,0}\right)\left(\Delta^{2} \sigma_{z}\right)_{k, j}=\frac{7}{18} \pi a^{3} .
\end{aligned}
$$

Substituting (3.144), (3.145) and (3.146) into the right-hand side of (3.143), the value of the second term of the right-hand side of (3.120) is

$$
\begin{aligned}
& \lim _{n \rightarrow \infty} \sum_{k=1}^{n} \sum_{j=1}^{k}\left[\sum_{i=1}^{j}\left(\frac{\Delta_{i}\left(X^{\mu}\right)\left[\Delta_{i}\left(x^{\gamma}\right)_{0, i}\right]}{\Delta_{i}\left(x^{\gamma}\right)_{0, i}} \Delta_{i}\left(x^{\gamma}\right)_{0, i}\right)\right. \\
& \left.\quad+\sum_{h=1}^{k}\left(\frac{\Delta_{h}\left(X^{\mu}\right)\left[\Delta_{h}\left(x^{\gamma}\right)_{h, j}\right]}{\Delta_{h}\left(x^{\gamma}\right)_{h, j}} \Delta_{h}\left(x^{\gamma}\right)_{h, j}\right)\right]\left(\Delta^{2} \sigma_{\mu}\right)_{k, j}=\frac{5}{6} \pi a^{3},
\end{aligned}
$$


where the indices are summed over $\gamma, \mu=1,2,3$. Using (3.112) in Definition 6, the sum of (3.142) and (3.147) is the value of the right-hand side of (3.119) as follows:

$$
\iiint_{D} \frac{\partial X^{\prime \prime \mu}}{\partial x^{\prime \prime \gamma}} d x^{\prime \prime \gamma} d^{2} \sigma_{\mu}=\frac{4}{3} \pi a^{3}
$$

where the indices are summed over $\gamma, \mu=1,2,3$. For the example of the volume of the sphere, the values of both sides of (3.119) are calculated to be (3.137) and (3.148).

\section{Integral in the 4D time-space}

The quadruple integral is demanded to derive the fundamental equations of the relativistic theories from the variational principle in physics. As the curl theorem of a triangular integral in the 4D time-space was derived in the previous paper [4], the divergence theorem of a triangular integral in the 4D time-space is derived in this paper. In the 3D space, the surface area is divided into $(3-1) !=2$ parts and each trapezium is divided into $3-1=2$ triangles. In the $4 \mathrm{D}$ time-space, the hyper-surface area is divided into $(4-1) !=6$ parts and each hyper-trapezium is divided into $4-1=3$ tetrahedra.

Let $h(t, x, y, z)=0$ be a piecewise smooth hyper-surface of the equation in the txyz-timespace, expressed in the Cartesian coordinates $(t, x, y, z) \in \mathbb{R}^{4}$. Suppose there are $(4-1) !=$ 6 kinds of triple sequences of points $\left\{P_{j, k, l}\right\},\left\{P_{j, l, k}\right\},\left\{P_{l, j, k}\right\},\left\{P_{l, k, j}\right\},\left\{P_{k, l, j}\right\}$ and $\left\{P_{k, j, l}\right\}$ for $l=0,1,2, \ldots, j$ and $j=0,1,2, \ldots, k$ and $k=0,1,2, \ldots, n$ on $h(t, x, y, z)=0$. In the previous section, $(3-1) !=2$ kinds of finite area element vectors $\overrightarrow{\Delta^{2} \sigma_{j, k}}$ and $\overrightarrow{\Delta^{2} \sigma_{k, j}}$ in the $3 \mathrm{D}$ space are respectively introduced as (3.1) and (3.4). In this section, we introduce $(4-1) !=6$ kinds of finite hyper-surface element vectors $\overrightarrow{\Delta^{3} V_{j, k, l}}, \overrightarrow{\Delta^{3} V_{j, l, k}}, \overrightarrow{\Delta^{3} V_{l, j, k}}, \overrightarrow{\Delta^{3} V_{l, k, j}}, \overrightarrow{\Delta^{3} V_{k, l, j}}$ and $\overrightarrow{\Delta^{3} V_{k, j, l}}$ in the 4D time-space.

\subsection{Six kinds of finite hyper-surface element vectors in the 4D time-space}

In the following, the Cartesian coordinates are denoted as $x^{0}=t, x^{1}=x, x^{2}=y$ and $x^{3}=z$. Before expressing the following formulae in tensor representation, the antisymmetric symbol in the $4 \mathrm{D}$ time-space is introduced as

$$
\varepsilon^{\alpha \beta \gamma \delta}=\varepsilon_{\alpha \beta \gamma \delta}= \begin{cases}+1 & \text { for even permutation of }\{0,1,2,3\} \\ -1 & \text { for odd permutation of }\{0,1,2,3\} \\ 0 & \text { otherwise }\end{cases}
$$

In the $4 \mathrm{D}$ time-space, $\frac{1}{3 !} \varepsilon_{\alpha \beta \gamma \mu} \varepsilon^{\alpha \beta \gamma \delta}=\delta_{\mu}^{\delta}$ holds for $\delta, \mu=0,1,2,3$, where the indices are summed over $\alpha, \beta, \gamma=0,1,2,3$.

1. The first finite hyper-surface element vector $\left(\Delta^{3} V_{\mu}\right)_{j, k, l} \equiv\left(\overrightarrow{\Delta^{3} V_{j, k, l}}\right)_{\mu}$ for $\mu=0,1,2,3$ and $l=1,2, \ldots, j$ and $j=1,2, \ldots, k$ and $k=1,2, \ldots, n$ is disintegrated into three parts,

$$
\left(\overrightarrow{\Delta^{3} V_{j, k, l}}\right)_{\mu}=\left(\overrightarrow{\Delta_{j}^{3} V_{j, k, l}}\right)_{\mu}+\left(\overrightarrow{\Delta_{k}^{3} V_{j, k, l}}\right)_{\mu}+\left(\overrightarrow{\Delta_{l}^{3} V_{j, k, l}}\right)_{\mu},
$$

where $\left(\overrightarrow{\Delta^{3} V_{j, k, l}}\right)_{\mu},\left(\overrightarrow{\left.\Delta_{j}^{3} V_{j, k, l}\right)_{\mu}},\left(\overrightarrow{\Delta_{k}^{3} V_{j, k, l}}\right)_{\mu}\right.$ and $\left(\overrightarrow{\Delta_{l}^{3} V_{j, k, l}}\right)_{\mu}$ are respectively $\mu$-components of $\overrightarrow{\Delta^{3} V_{j, k, l}}, \overrightarrow{\Delta_{j}^{3} V_{j, k, l}}, \overrightarrow{\Delta_{k}^{3} V_{j, k, l}}$ and $\overrightarrow{\Delta_{l}^{3} V_{j, k, l}}$. Here, $\left(\overrightarrow{\Delta_{j}^{3} V_{j, k, l}}\right)_{\mu},\left(\overrightarrow{\Delta_{k}^{3} V_{j, k, l}}\right)_{\mu}$ and $\left(\overrightarrow{\Delta_{l}^{3} V_{j, k, l}}\right)_{\mu}$ for $\mu=$ 
$0,1,2,3$ and $l=1,2, \ldots, j$ and $j=1,2, \ldots, k$ and $k=1,2, \ldots, n$ are respectively inferred as

$$
\begin{aligned}
\left(\overrightarrow{\Delta_{j}^{3} V_{j, k, l}}\right)_{\mu} & =\frac{1}{3 !} \varepsilon_{\alpha \beta \gamma \mu}\left(\overrightarrow{P_{j, k, l} P_{j-1, k, l}}\right)^{\alpha}\left(\overrightarrow{P_{j, k, l} P_{j-1, k-1, l}}\right)^{\beta}\left(\overrightarrow{P_{j, k, l} P_{j-1, k-1, l-1}}\right)^{\gamma}, \\
\left(\overrightarrow{\Delta_{k}^{3} V_{j, k, l}}\right)_{\mu} & =\frac{1}{3 !} \varepsilon_{\alpha \beta \gamma \mu}\left(\overrightarrow{P_{j, k, l} P_{j-1, k-1, l-1}}\right)^{\alpha}\left(\overrightarrow{P_{j, k, l} P_{j, k-1, l}}\right)^{\beta}\left(\overrightarrow{P_{j, k, l} P_{j, k-1, l-1}}\right)^{\gamma}, \\
\left(\overrightarrow{\Delta_{l}^{3} V_{j, k, l}}\right)_{\mu} & =\frac{1}{3 !} \varepsilon_{\alpha \beta \gamma \mu}\left(\overrightarrow{P_{j, k, l} P_{j-1, k, l-1}}\right)^{\alpha}\left(\overrightarrow{P_{j, k, l} P_{j-1, k-1, l-1}}\right)^{\beta}\left(\overrightarrow{P_{j, k, l} P_{j, k, l-1}}\right)^{\gamma},
\end{aligned}
$$

where the indices are summed over $\alpha, \beta, \gamma=0,1,2,3$.

(a) Introducing

$$
\begin{aligned}
& \Delta_{j}\left(x^{\mu}\right)_{j, k, l} \equiv\left(x^{\mu}\right)_{j, k, l}-\left(x^{\mu}\right)_{j-1, k, l}, \\
& \Delta_{k}\left(x^{\mu}\right)_{j-1, k, l} \equiv\left(x^{\mu}\right)_{j-1, k, l}-\left(x^{\mu}\right)_{j-1, k-1, l}, \\
& \Delta_{l}\left(x^{\mu}\right)_{j-1, k-1, l} \equiv\left(x^{\mu}\right)_{j-1, k-1, l}-\left(x^{\mu}\right)_{j-1, k-1, l-1}
\end{aligned}
$$

for $\alpha, \beta, \gamma, \mu=0,1,2,3$ and $l=1,2, \ldots, j$ and $j=1,2, \ldots, k$ and $k=1,2, \ldots, n$, we may modify each factor of (4.3) as follows:

$$
\begin{aligned}
\left(\overrightarrow{P_{j, k, l} P_{j-1, k, l}}\right)^{\alpha}= & \left(\overrightarrow{O P_{j-1, k, l}}\right)^{\alpha}-\left(\overrightarrow{O P_{j, k, l}}\right)^{\alpha} \\
= & \left(x^{\alpha}\right)_{j-1, k, l}-\left(x^{\alpha}\right)_{j, k, l} \\
= & -\Delta_{j}\left(x^{\alpha}\right)_{j, k, l}, \\
\left(\overrightarrow{P_{j, k, l} P_{j-1, k-1, l}}\right)^{\beta}= & \left(\overrightarrow{O P_{j-1, k-1, l}}\right)^{\beta}-\left(\overrightarrow{O P_{j, k, l}}\right)^{\beta} \\
= & \left(x^{\beta}\right)_{j-1, k-1, l}-\left(x^{\beta}\right)_{j, k, l} \\
= & -\Delta_{j}\left(x^{\beta}\right)_{j, k, l}-\Delta_{k}\left(x^{\beta}\right)_{j-1, k, l} \\
\left(\overrightarrow{P_{j, k, l} P_{j-1, k-1, l-1}}\right)^{\gamma} & =\left(\overrightarrow{O P_{j-1, k-1, l-1}}\right)^{\gamma}-\left(\overrightarrow{O P_{j, k, l}}\right)^{\gamma} \\
& =\left(x^{\gamma}\right)_{j-1, k-1, l-1}-\left(x^{\gamma}\right)_{j, k, l} \\
& =-\Delta_{j}\left(x^{\gamma}\right)_{j, k, l}-\Delta_{k}\left(x^{\gamma}\right)_{j-1, k, l}-\Delta_{l}\left(x^{\gamma}\right)_{j-1, k-1, l}
\end{aligned}
$$

Substituting (4.9), (4.10) and (4.11) in (4.3) for $\mu=0,1,2,3$ and $l=1,2, \ldots, j$ and $j=$ $1,2, \ldots, k$ and $k=1,2, \ldots, n$, we may modify it as

$$
\begin{aligned}
\left(\overrightarrow{\Delta_{j}^{3} V_{j, k, l}}\right)_{\mu}= & -\frac{1}{3 !} \varepsilon_{\alpha \beta \gamma \mu} \Delta_{j}\left(x^{\alpha}\right)_{j, k, l}\left[\Delta_{j}\left(x^{\beta}\right)_{j, k, l}+\Delta_{k}\left(x^{\beta}\right)_{j-1, k, l}\right] \\
& \times\left[\Delta_{j}\left(x^{\gamma}\right)_{j, k, l}+\Delta_{k}\left(x^{\gamma}\right)_{j-1, k, l}+\Delta_{l}\left(x^{\gamma}\right)_{j-1, k-1, l}\right],
\end{aligned}
$$

where the indices are summed over $\alpha, \beta, \gamma=0,1,2,3$.

(b) In the similar manner, (4.4) for $\mu=0,1,2,3$ and $l=1,2, \ldots, j$ and $j=1,2, \ldots, k$ and $k=1,2, \ldots, n$ is modified to be

$$
\begin{aligned}
\left(\overrightarrow{\Delta_{k}^{3} V_{j, k, l}}\right)_{\mu}= & -\frac{1}{3 !} \varepsilon_{\alpha \beta \gamma \mu} \Delta_{k}\left(x^{\beta}\right)_{j, k, l}\left[\Delta_{k}\left(x^{\gamma}\right)_{j, k, l}+\Delta_{l}\left(x^{\gamma}\right)_{j, k-1, l}\right] \\
& \times\left[\Delta_{k}\left(x^{\alpha}\right)_{j, k, l}+\Delta_{l}\left(x^{\alpha}\right)_{j, k-1, l}+\Delta_{j}\left(x^{\alpha}\right)_{j, k-1, l-1}\right]
\end{aligned}
$$

where the indices are summed over $\alpha, \beta, \gamma=0,1,2,3$. 
(c) In the similar manner, (4.5) for $\mu=0,1,2,3$ and $l=1,2, \ldots, j$ and $j=1,2, \ldots, k$ and $k=1,2, \ldots, n$ is modified to be

$$
\begin{aligned}
\left(\overrightarrow{\Delta_{l}^{3} V_{j, k, l}}\right)_{\mu}= & -\frac{1}{3 !} \varepsilon_{\alpha \beta \gamma \mu} \Delta_{l}\left(x^{\gamma}\right)_{j, k, l}\left[\Delta_{l}\left(x^{\alpha}\right)_{j, k, l}+\Delta_{j}\left(x^{\alpha}\right)_{j, k, l-1}\right] \\
& \times\left[\Delta_{l}\left(x^{\beta}\right)_{j, k, l}+\Delta_{j}\left(x^{\beta}\right)_{j, k, l-1}+\Delta_{k}\left(x^{\beta}\right)_{j-1, k, l-1}\right]
\end{aligned}
$$

where the indices are summed over $\alpha, \beta, \gamma=0,1,2,3$.

Substituting (4.12), (4.13) and (4.14) into (4.2) for $\mu=0,1,2,3$ and $l=1,2, \ldots, j$ and $j=$ $1,2, \ldots, k$ and $k=1,2, \ldots, n$, we may modify it as

$$
\begin{aligned}
\left(\Delta^{3} V_{\mu}\right)_{j, k, l}= & -\frac{1}{3 !} \varepsilon_{\alpha \beta \gamma \mu}\left\{\Delta_{j}\left(x^{\alpha}\right)_{j, k, l}\left[\Delta_{j}\left(x^{\beta}\right)_{j, k, l}+\Delta_{k}\left(x^{\beta}\right)_{j-1, k, l}\right]\right. \\
& \times\left[\Delta_{j}\left(x^{\gamma}\right)_{j, k, l}+\Delta_{k}\left(x^{\gamma}\right)_{j-1, k, l}+\Delta_{l}\left(x^{\gamma}\right)_{j-1, k-1, l}\right] \\
& +\Delta_{k}\left(x^{\beta}\right)_{j, k, l}\left[\Delta_{k}\left(x^{\gamma}\right)_{j, k, l}+\Delta_{l}\left(x^{\gamma}\right)_{j, k-1, l}\right] \\
& \times\left[\Delta_{k}\left(x^{\alpha}\right)_{j, k, l}+\Delta_{l}\left(x^{\alpha}\right)_{j, k-1, l}+\Delta_{j}\left(x^{\alpha}\right)_{j, k-1, l-1}\right] \\
& +\Delta_{l}\left(x^{\gamma}\right)_{j, k, l}\left[\Delta_{l}\left(x^{\alpha}\right)_{j, k, l}+\Delta_{j}\left(x^{\alpha}\right)_{j, k, l-1}\right] \\
& \left.\times\left[\Delta_{l}\left(x^{\beta}\right)_{j, k, l}+\Delta_{j}\left(x^{\beta}\right)_{j, k, l-1}+\Delta_{k}\left(x^{\beta}\right)_{j-1, k, l-1}\right]\right\}
\end{aligned}
$$

where the indices are summed over $\alpha, \beta, \gamma=0,1,2,3$.

In the similar manner, we calculate the case of $(j, l, k),(k, j, l),(k, l, j),(l, k, j)$ and $(l, j, k)$ as follows.

2. The second finite hyper-surface element vector $\left(\Delta^{3} V_{\mu}\right)_{j, l, k} \equiv\left(\overrightarrow{\Delta^{3} V_{j, l, k}}\right)_{\mu}$ for $\mu=$ $0,1,2,3$ and $l=1,2, \ldots, j$ and $j=1,2, \ldots, k$ and $k=1,2, \ldots, n$ is

$$
\begin{aligned}
\left(\Delta^{3} V_{\mu}\right)_{j, l, k}= & -\frac{1}{3 !} \varepsilon_{\alpha \beta \gamma \mu}\left\{\Delta_{j}\left(x^{\alpha}\right)_{j, l, k}\left[\Delta_{j}\left(x^{\beta}\right)_{j, l, k}+\Delta_{l}\left(x^{\beta}\right)_{j-1, l, k}\right]\right. \\
& \times\left[\Delta_{j}\left(x^{\gamma}\right)_{j, l, k}+\Delta_{l}\left(x^{\gamma}\right)_{j-1, l, k}+\Delta_{k}\left(x^{\gamma}\right)_{j-1, l-1, k}\right] \\
& +\Delta_{l}\left(x^{\beta}\right)_{j, l, k}\left[\Delta_{l}\left(x^{\gamma}\right)_{j, l, k}+\Delta_{k}\left(x^{\gamma}\right)_{j, l-1, k}\right] \\
& \times\left[\Delta_{l}\left(x^{\alpha}\right)_{j, l, k}+\Delta_{k}\left(x^{\alpha}\right)_{j, l-1, k}+\Delta_{j}\left(x^{\alpha}\right)_{j, l-1, k-1}\right] \\
& +\Delta_{k}\left(x^{\gamma}\right)_{j, l, k}\left[\Delta_{k}\left(x^{\alpha}\right)_{j, l, k}+\Delta_{j}\left(x^{\alpha}\right)_{j, l, k-1}\right] \\
& \left.\times\left[\Delta_{k}\left(x^{\beta}\right)_{j, l, k}+\Delta_{j}\left(x^{\beta}\right)_{j, l, k-1}+\Delta_{l}\left(x^{\beta}\right)_{j-1, l, k-1}\right]\right\},
\end{aligned}
$$

where the indices are summed over $\alpha, \beta, \gamma=0,1,2,3$.

3. The third finite hyper-surface element vector $\left(\Delta^{3} V_{\mu}\right)_{l, j, k} \equiv\left(\overrightarrow{\Delta^{3} V_{l, j, k}}\right)_{\mu}$ for $\mu=0,1,2,3$ and $l=1,2, \ldots, j$ and $j=1,2, \ldots, k$ and $k=1,2, \ldots, n$ is

$$
\begin{aligned}
\left(\Delta^{3} V_{\mu}\right)_{l, j, k}= & -\frac{1}{3 !} \varepsilon_{\alpha \beta \gamma \mu}\left\{\Delta_{l}\left(x^{\alpha}\right)_{l, j, k}\left[\Delta_{l}\left(x^{\beta}\right)_{l, j, k}+\Delta_{j}\left(x^{\beta}\right)_{l-1, j, k}\right]\right. \\
& \times\left[\Delta_{l}\left(x^{\gamma}\right)_{l, j, k}+\Delta_{j}\left(x^{\gamma}\right)_{l-1, j, k}+\Delta_{k}\left(x^{\gamma}\right)_{l-1, j-1, k}\right] \\
& +\Delta_{j}\left(x^{\beta}\right)_{l, j, k}\left[\Delta_{j}\left(x^{\gamma}\right)_{l, j, k}+\Delta_{k}\left(x^{\gamma}\right)_{l, j-1, k}\right] \\
& \times\left[\Delta_{j}\left(x^{\alpha}\right)_{l, j, k}+\Delta_{k}\left(x^{\alpha}\right)_{l, j-1, k}+\Delta_{l}\left(x^{\alpha}\right)_{l, j-1, k-1}\right]
\end{aligned}
$$


Tokunaga Advances in Difference Equations 2012, 2012:168

Page 37 of 59

http://www.advancesindifferenceequations.com/content/2012/1/168

$$
\begin{aligned}
& +\Delta_{k}\left(x^{\gamma}\right)_{l, j, k}\left[\Delta_{k}\left(x^{\alpha}\right)_{l, j, k}+\Delta_{l}\left(x^{\alpha}\right)_{l, j, k-1}\right] \\
& \left.\times\left[\Delta_{k}\left(x^{\beta}\right)_{l, j, k}+\Delta_{l}\left(x^{\beta}\right)_{l, j, k-1}+\Delta_{j}\left(x^{\beta}\right)_{l-1, j, k-1}\right]\right\},
\end{aligned}
$$

where the indices are summed over $\alpha, \beta, \gamma=0,1,2,3$.

4. The fourth finite hyper-surface element vector $\left(\Delta^{3} V_{\mu}\right)_{l, k, j} \equiv\left(\overrightarrow{\Delta^{3} V_{l, k, j}}\right)_{\mu}$ for $\mu=0,1,2,3$ and $l=1,2, \ldots, j$ and $j=1,2, \ldots, k$ and $k=1,2, \ldots, n$ is

$$
\begin{aligned}
\left(\Delta^{3} V_{\mu}\right)_{l, k, j}= & -\frac{1}{3 !} \varepsilon_{\alpha \beta \gamma \mu}\left\{\Delta_{l}\left(x^{\alpha}\right)_{l, k, j}\left[\Delta_{l}\left(x^{\beta}\right)_{l, k, j}+\Delta_{k}\left(x^{\beta}\right)_{l-1, k, j}\right]\right. \\
& \times\left[\Delta_{l}\left(x^{\gamma}\right)_{l, k, j}+\Delta_{k}\left(x^{\gamma}\right)_{l-1, k, j}+\Delta_{j}\left(x^{\gamma}\right)_{l-1, k-1, j}\right] \\
& +\Delta_{k}\left(x^{\beta}\right)_{l, k, j}\left[\Delta_{k}\left(x^{\gamma}\right)_{l, k, j}+\Delta_{j}\left(x^{\gamma}\right)_{l, k-1, j}\right] \\
& \times\left[\Delta_{k}\left(x^{\alpha}\right)_{l, k, j}+\Delta_{j}\left(x^{\alpha}\right)_{l, k-1, j}+\Delta_{l}\left(x^{\alpha}\right)_{l, k-1, j-1}\right] \\
& +\Delta_{j}\left(x^{\gamma}\right)_{l, k, j}\left[\Delta_{j}\left(x^{\alpha}\right)_{l, k, j}+\Delta_{l}\left(x^{\alpha}\right)_{l, k, j-1}\right] \\
& \left.\times\left[\Delta_{j}\left(x^{\beta}\right)_{l, k, j}+\Delta_{l}\left(x^{\beta}\right)_{l, k, j-1}+\Delta_{k}\left(x^{\beta}\right)_{l-1, k, j-1}\right]\right\},
\end{aligned}
$$

where the indices are summed over $\alpha, \beta, \gamma=0,1,2,3$.

5. The fifth finite hyper-surface element vector $\left(\Delta^{3} V_{\mu}\right)_{k, l, j} \equiv\left(\overrightarrow{\Delta^{3} V_{k, l, j}}\right)_{\mu}$ for $\mu=0,1,2,3$ and $l=1,2, \ldots, j$ and $j=1,2, \ldots, k$ and $k=1,2, \ldots, n$ is

$$
\begin{aligned}
\left(\Delta^{3} V_{\mu}\right)_{k, l, j}= & -\frac{1}{3 !} \varepsilon_{\alpha \beta \gamma \mu}\left\{\Delta_{k}\left(x^{\alpha}\right)_{k, l, j}\left[\Delta_{k}\left(x^{\beta}\right)_{k, l, j}+\Delta_{l}\left(x^{\beta}\right)_{k-1, l, j}\right]\right. \\
& \times\left[\Delta_{k}\left(x^{\gamma}\right)_{k, l, j}+\Delta_{l}\left(x^{\gamma}\right)_{k-1, l, j}+\Delta_{j}\left(x^{\gamma}\right)_{k-1, l-1, j}\right] \\
& +\Delta_{l}\left(x^{\beta}\right)_{k, l, j}\left[\Delta_{l}\left(x^{\gamma}\right)_{k, l, j}+\Delta_{j}\left(x^{\gamma}\right)_{k, l-1, j}\right] \\
& \times\left[\Delta_{l}\left(x^{\alpha}\right)_{k, l, j}+\Delta_{j}\left(x^{\alpha}\right)_{k, l-1, j}+\Delta_{k}\left(x^{\alpha}\right)_{k, l-1, j-1}\right] \\
& +\Delta_{j}\left(x^{\gamma}\right)_{k, l, j}\left[\Delta_{j}\left(x^{\alpha}\right)_{k, l, j}+\Delta_{k}\left(x^{\alpha}\right)_{k, l, j-1}\right] \\
& \left.\times\left[\Delta_{j}\left(x^{\beta}\right)_{k, l, j}+\Delta_{k}\left(x^{\beta}\right)_{k, l, j-1}+\Delta_{l}\left(x^{\beta}\right)_{k-1, l, j-1}\right]\right\}
\end{aligned}
$$

where the indices are summed over $\alpha, \beta, \gamma=0,1,2,3$.

6. The sixth finite hyper-surface element vector $\left(\Delta^{3} V_{\mu}\right)_{k, j, l} \equiv\left(\overrightarrow{\Delta^{3} V_{k, j, l}}\right)_{\mu}$ for $\mu=0,1,2,3$ and $l=1,2, \ldots, j$ and $j=1,2, \ldots, k$ and $k=1,2, \ldots, n$ is

$$
\begin{aligned}
\left(\Delta^{3} V_{\mu}\right)_{k, j, l}= & -\frac{1}{3 !} \varepsilon_{\alpha \beta \gamma \mu}\left\{\Delta_{k}\left(x^{\alpha}\right)_{k, j, l}\left[\Delta_{k}\left(x^{\beta}\right)_{k, j, l}+\Delta_{j}\left(x^{\beta}\right)_{k-1, j, l}\right]\right. \\
& \times\left[\Delta_{k}\left(x^{\gamma}\right)_{k, j, l}+\Delta_{j}\left(x^{\gamma}\right)_{k-1, j, l}+\Delta_{l}\left(x^{\gamma}\right)_{k-1, j-1, l}\right] \\
& +\Delta_{j}\left(x^{\beta}\right)_{k, j, l}\left[\Delta_{j}\left(x^{\gamma}\right)_{k, j, l}+\Delta_{l}\left(x^{\gamma}\right)_{k, j-1, l}\right] \\
& \times\left[\Delta_{j}\left(x^{\alpha}\right)_{k, j, l}+\Delta_{l}\left(x^{\alpha}\right)_{k, j-1, l}+\Delta_{k}\left(x^{\alpha}\right)_{k, j-1, l-1}\right] \\
& +\Delta_{l}\left(x^{\gamma}\right)_{k, j, l}\left[\Delta_{l}\left(x^{\alpha}\right)_{k, j, l}+\Delta_{k}\left(x^{\alpha}\right)_{k, j, l-1}\right] \\
& \left.\times\left[\Delta_{l}\left(x^{\beta}\right)_{k, j, l}+\Delta_{k}\left(x^{\beta}\right)_{k, j, l-1}+\Delta_{j}\left(x^{\beta}\right)_{k-1, j, l-1}\right]\right\},
\end{aligned}
$$

where the indices are summed over $\alpha, \beta, \gamma=0,1,2,3$. 
Proposition 4 The volume $V$ of the $3 D$ hyper-surface of the $4 D$ hyper-body in $\mathbb{R}^{4}$ is expressed as

$$
\begin{aligned}
V= & \lim _{n \rightarrow \infty} \sum_{k=1}^{n} \sum_{j=1}^{k} \sum_{l=1}^{j}\left(\overrightarrow{n_{j, k, l}} \cdot \overrightarrow{\Delta^{3} V_{j, k, l}}+\overrightarrow{n_{j, l, k}} \cdot \overrightarrow{\Delta^{3} V_{j, l, k}}+\overrightarrow{n_{l, j, k}} \cdot \overrightarrow{\Delta^{3} V_{l, j, k}}\right. \\
& \left.+\overrightarrow{n_{l, k, j}} \cdot \overrightarrow{\Delta^{3} V_{l, k, j}}+\overrightarrow{n_{k, l, j}} \cdot \overrightarrow{\Delta^{3} V_{k, l, j}}+\overrightarrow{n_{k, j, l}} \cdot \overrightarrow{\Delta^{3} V_{k, j, l}}\right)
\end{aligned}
$$

under the condition of

$$
\lim _{n \rightarrow \infty} \sum_{k=1}^{n} \overrightarrow{n_{k, k, k}} \cdot \overrightarrow{\Delta^{3} V_{k, k, k}}=0
$$

where $\overrightarrow{\Delta^{3} V_{j, k, l}}, \overrightarrow{\Delta^{3} V_{j, l, k}}, \overrightarrow{\Delta^{3} V_{l, j, k}}, \overrightarrow{\Delta^{3} V_{l, k, j}}, \overrightarrow{\Delta^{3} V_{k, l, j}}$ and $\overrightarrow{\Delta^{3} V_{k, j, l}}$ are the finite hyper-surface elements and $\overrightarrow{n_{j, l, k}}, \overrightarrow{n_{j, l, k}}, \overrightarrow{n_{l, j, k}}, \overrightarrow{n_{l, k, j}}, \overrightarrow{n_{k, l, j}}$ and $\overrightarrow{n_{k, j, l}}$ are corresponding unit normal vectors of respective finite hyper-surface elements.

\subsection{The divergence theorem of a triangular integral in the 4D time-space}

In the following subsection, we prepare definitions and notations for extending Theorem 3 and Corollary 3 in the 4D time-space. Assume there are four functions $T=T(t, x, y, z)$, $X=X(t, x, y, z), Y=Y(t, x, y, z)$ and $Z=Z(t, x, y, z)$ of the four variables $t, x, y$ and $z$ in the Cartesian coordinates of the $4 \mathrm{D}$ time-space. Suppose there are $(4-1) !=6$ sets of triple dependent sequences (4.23), (4.24), (4.25), (4.26), (4.27) and (4.28) as follows. Denoting $X^{0}=T, X^{1}=X, X^{2}=Y$ and $X^{3}=Z$, the $(4-1) !=6$ kinds of integrand vectors $\overrightarrow{W_{j, k, l}}, \overrightarrow{W_{j, l, k}}$, $\overrightarrow{W_{l, j, k}}, \overrightarrow{W_{l, k, j}}, \overrightarrow{W_{k, l, j}}$ and $\overrightarrow{W_{k, j, l}}$ for $\mu=0,1,2,3$ and $l=0,1,2, \ldots, j$ and $j=0,1,2, \ldots, k$ and $k=0,1,2, \ldots, n$ are expressed as

$$
\begin{aligned}
& \overrightarrow{W_{j, k, l}} \equiv\left(X^{\mu}\right)_{j, k, l}=\left(T_{j, k, l}, X_{j, k, l}, Y_{j, k, l}, Z_{j, k, l}\right), \\
& \overrightarrow{W_{j, l, k}} \equiv\left(X^{\mu}\right)_{j, l, k}=\left(T_{j, l, k}, X_{j, l, k}, Y_{j, l, k}, Z_{j, l, k}\right), \\
& \overrightarrow{W_{l, j, k}} \equiv\left(X^{\mu}\right)_{l, j, k}=\left(T_{l, j, k}, X_{l, j, k}, Y_{l, j, k}, Z_{l, j, k}\right), \\
& \overrightarrow{W_{l, k, j}} \equiv\left(X^{\mu}\right)_{l, k, j}=\left(T_{l, k, j}, X_{l, k, j}, Y_{l, k, j}, Z_{l, k, j}\right), \\
& \overrightarrow{W_{k, l, j}} \equiv\left(X^{\mu}\right)_{k, l, j}=\left(T_{k, l, j}, X_{k, l, j}, Y_{k, l, j}, Z_{k, l, j}\right), \\
& \overrightarrow{W_{k, j, l}} \equiv\left(X^{\mu}\right)_{k, j, l}=\left(T_{k, j, l}, X_{k, j, l}, Y_{k, j, l}, Z_{k, j, l}\right) .
\end{aligned}
$$

Replacing a normal vector $\vec{n}$ in the 4D time-space by a general vector $\vec{W}=\left(X^{\mu}\right)=$ $\left(X^{0}, X^{1}, X^{2}, X^{3}\right)=(T, X, Y, Z)$ in the $4 \mathrm{D}$ time-space, where $X^{\mu}=X^{\mu}\left(x^{\alpha}\right)$, we introduce the following triple dependent sums:

$$
\begin{aligned}
\sum_{k=1}^{n} & \sum_{j=1}^{k} \sum_{l=1}^{j}\left(\overrightarrow{W_{j, k, l}} \cdot \overrightarrow{\Delta^{3} V_{j, k, l}}+\overrightarrow{W_{j, l, k}} \cdot \overrightarrow{\Delta^{3} V_{j, l, k}}+\overrightarrow{W_{l, j, k}} \cdot \overrightarrow{\Delta^{3} V_{l, j, k}}\right. \\
& \left.+\overrightarrow{W_{l, k, j}} \cdot \overrightarrow{\Delta^{3} V_{l, k, j}}+\overrightarrow{W_{k, l, j}} \cdot \overrightarrow{\Delta^{3} V_{k, l, j}}+\overrightarrow{W_{k, j, l}} \cdot \overrightarrow{\Delta^{3} V_{k, j, l}}\right)
\end{aligned}
$$




$$
\begin{aligned}
\equiv & \sum_{k=1}^{n} \sum_{j=1}^{k} \sum_{l=1}^{j}\left[\left(X^{\mu}\right)_{j, k, l}\left(\Delta^{3} V_{\mu}\right)_{j, k, l}+\left(X^{\mu}\right)_{j, l, k}\left(\Delta^{3} V_{\mu}\right)_{j, l, k}+\left(X^{\mu}\right)_{l, j, k}\left(\Delta^{3} V_{\mu}\right)_{l, j, k}\right. \\
& \left.+\left(X^{\mu}\right)_{l, k, j}\left(\Delta^{3} V_{\mu}\right)_{l, k, j}+\left(X^{\mu}\right)_{k, l, j}\left(\Delta^{3} V_{\mu}\right)_{k, l, j}+\left(X^{\mu}\right)_{k, j, l}\left(\Delta^{3} V_{\mu}\right)_{k, j, l}\right]
\end{aligned}
$$

where the index is summed over $\mu=0,1,2,3$. In order to prove Theorem $4,\left(X^{\mu}\right)_{j, k, l}$, $\left(X^{\mu}\right)_{j, l, k},\left(X^{\mu}\right)_{l, j, k},\left(X^{\mu}\right)_{l, k, j,},\left(X^{\mu}\right)_{k, l, j}$ and $\left(X^{\mu}\right)_{k, j, l}$ are respectively modified in Lemmata 6,7 , $8,9,10$ and 11 .

1. The total increments of $\left(X^{\mu}\right)_{0,0, m}$ for $\mu=0,1,2,3$ and $m=1,2, \ldots, l$ are denoted as

$$
\begin{aligned}
\Delta_{m}\left(X^{\mu}\right)_{0,0, m} \equiv & \left(X^{\mu}\right)_{0,0, m}-\left(X^{\mu}\right)_{0,0, m-1} \\
= & X^{\mu}\left(t_{0,0, m}, x_{0,0, m}, y_{0,0, m}, z_{0,0, m}\right) \\
& -X^{\mu}\left(t_{0,0, m-1}, x_{0,0, m-1}, y_{0,0, m-1}, z_{0,0, m-1}\right) .
\end{aligned}
$$

The increments of $\Delta_{m}\left(x^{\delta}\right)_{0,0, m}$ for $\delta=0,1,2,3$ and $m=1,2, \ldots, l$ are denoted as

$$
\Delta_{m}\left(x^{\delta}\right)_{0,0, m} \equiv\left(x^{\delta}\right)_{0,0, m}-\left(x^{\delta}\right)_{0,0, m-1} .
$$

The partial increments of $\Delta_{m}\left(X^{\mu}\right)_{0,0, m}$ for $\mu=0,1,2,3$ and $m=1,2, \ldots, l$ are denoted as

$$
\begin{aligned}
\Delta_{m}\left(X^{\mu}\right)\left[\Delta_{m} t_{0,0, m}\right] \equiv & X^{\mu}\left(t_{0,0, m}, x_{0,0, m}, y_{0,0, m}, z_{0,0, m}\right) \\
& -X^{\mu}\left(t_{0,0, m-1}, x_{0,0, m}, y_{0,0, m}, z_{0,0, m}\right), \\
\Delta_{m}\left(X^{\mu}\right)\left[\Delta_{m} x_{0,0, m}\right] \equiv & X^{\mu}\left(t_{0,0, m-1}, x_{0,0, m}, y_{0,0, m}, z_{0,0, m}\right) \\
& -X^{\mu}\left(t_{0,0, m-1}, x_{0,0, m-1}, y_{0,0, m}, z_{0,0, m}\right), \\
\Delta_{m}\left(X^{\mu}\right)\left[\Delta_{m} y_{0,0, m}\right] \equiv & X^{\mu}\left(t_{0,0, m-1}, x_{0,0, m-1}, y_{0,0, m}, z_{0,0, m}\right) \\
& -X^{\mu}\left(t_{0,0, m-1}, x_{0,0, m-1}, y_{0,0, m-1}, z_{0,0, m}\right), \\
\Delta_{m}\left(X^{\mu}\right)\left[\Delta_{m} z_{0,0, m}\right] \equiv & X^{\mu}\left(t_{0,0, m-1}, x_{0,0, m-1}, y_{0,0, m-1}, z_{0,0, m}\right) \\
& -X^{\mu}\left(t_{0,0, m-1}, x_{0,0, m-1}, y_{0,0, m-1}, z_{0,0, m-1}\right) .
\end{aligned}
$$

2. The total increments of $\left(X^{\mu}\right)_{i, 0, l}$ for $\mu=0,1,2,3$ and $i, l=1,2, \ldots, j$ are denoted as

$$
\begin{aligned}
\Delta_{i}\left(X^{\mu}\right)_{i, 0, l} & \equiv\left(X^{\mu}\right)_{i, 0, l}-\left(X^{\mu}\right)_{i-1,0, l} \\
& =X^{\mu}\left(t_{i, 0, l}, x_{i, 0, l}, y_{i, 0, l}, z_{i, 0, l}\right)-X^{\mu}\left(t_{i-1,0, l}, x_{i-1,0, l}, y_{i-1,0, l}, z_{i-1,0, l}\right) .
\end{aligned}
$$

The increments of $\Delta_{i}\left(x^{\delta}\right)_{i, 0, l}$ for $\delta=0,1,2,3$ and $i, l=1,2, \ldots, j$ are denoted as

$$
\Delta_{i}\left(x^{\delta}\right)_{i, 0, l} \equiv\left(x^{\delta}\right)_{i, 0, l}-\left(x^{\delta}\right)_{i-1,0, l^{*}}
$$

The partial increments of $\Delta_{i}\left(X^{\mu}\right)_{i, 0, l}$ for $\mu=0,1,2,3$ and $i, l=1,2, \ldots, j$ are denoted as

$$
\begin{aligned}
& \Delta_{i}\left(X^{\mu}\right)\left[\Delta_{i} t_{i, 0, l}\right] \equiv X^{\mu}\left(t_{i, 0, l}, x_{i, 0, l}, y_{i, 0, l}, z_{i, 0, l}\right)-X^{\mu}\left(t_{i-1,0, l}, x_{i, 0, l}, y_{i, 0, l}, z_{i, 0, l}\right), \\
& \Delta_{i}\left(X^{\mu}\right)\left[\Delta_{i} x_{i, 0, l}\right] \equiv X^{\mu}\left(t_{i-1,0, l}, x_{i, 0, l}, y_{i, 0, l}, z_{i, 0, l}\right)-X^{\mu}\left(t_{i-1,0, l}, x_{i-1,0, l}, y_{i, 0, l}, z_{i, 0, l}\right),
\end{aligned}
$$




$$
\begin{aligned}
\Delta_{i}\left(X^{\mu}\right)\left[\Delta_{i} y_{i, 0, l}\right] \equiv & X^{\mu}\left(t_{i-1,0, l}, x_{i-1,0, l}, y_{i, 0, l}, z_{i, 0, l}\right) \\
& -X^{\mu}\left(t_{i-1,0, l}, x_{i-1,0, l}, y_{i-1,0, l}, z_{i, 0, l}\right), \\
\Delta_{i}\left(X^{\mu}\right)\left[\Delta_{i} z_{i, 0, l}\right] \equiv & X^{\mu}\left(t_{i-1,0, l}, x_{i-1,0, l}, y_{i-1,0, l}, z_{i, 0, l}\right) \\
& -X^{\mu}\left(t_{i-1,0, l}, x_{i-1,0, l}, y_{i-1,0, l}, z_{i-1,0, l}\right) .
\end{aligned}
$$

3. The total increments of $\left(X^{\mu}\right)_{j, h, l}$ for $\mu=0,1,2,3$ and $l=1,2, \ldots, j$ and $h, j=1,2, \ldots, k$ are denoted as

$$
\begin{aligned}
\Delta_{h}\left(X^{\mu}\right)_{j, h, l} & \equiv\left(X^{\mu}\right)_{j, h, l}-\left(X^{\mu}\right)_{j, h-1, l} \\
& =X^{\mu}\left(t_{j, h, l}, x_{j, h, l}, y_{j, h, l}, z_{j, h, l}\right)-X^{\mu}\left(t_{j, h-1, l}, x_{j, h-1, l}, y_{j, h-1, l}, z_{j, h-1, l}\right) .
\end{aligned}
$$

The increments of $\left(x^{\delta}\right)_{j, h, l}$ for $\delta=0,1,2,3$ and $l=1,2, \ldots, j$ and $h, j=1,2, \ldots, k$ are denoted as

$$
\Delta_{h}\left(x^{\delta}\right)_{j, h, l} \equiv\left(x^{\delta}\right)_{j, h, l}-\left(x^{\delta}\right)_{j, h-1, l}
$$

The partial increments of $\left(X^{\mu}\right)_{j, h, l}$ for $\mu=0,1,2,3$ and $l=1,2, \ldots, j$ and $h, j=1,2, \ldots, k$ are denoted as

$$
\begin{aligned}
\Delta_{h}\left(X^{\mu}\right)\left[\Delta_{h} t_{j, h, l}\right] \equiv & X^{\mu}\left(t_{j, h, l}, x_{j, h, l}, y_{j, h, l}, z_{j, h, l}\right)-X^{\mu}\left(t_{j, h-1, l}, x_{j, h, l}, y_{j, h, l}, z_{j, h, l}\right), \\
\Delta_{h}\left(X^{\mu}\right)\left[\Delta_{h} x_{j, h, l}\right] \equiv & X^{\mu}\left(t_{j, h-1, l}, x_{j, h, l}, y_{j, h, l}, z_{j, h, l}\right)-X^{\mu}\left(t_{j, h-1, l}, x_{j, h-1, l}, y_{j, h, l}, z_{j, h, l}\right), \\
\Delta_{h}\left(X^{\mu}\right)\left[\Delta_{h} y_{j, h, l}\right] \equiv & X^{\mu}\left(t_{j, h-1, l}, x_{j, h-1, l}, y_{j, h, l}, z_{j, h, l}\right) \\
& -X^{\mu}\left(t_{j, h-1, l}, x_{j, h-1, l}, y_{j, h-1, l}, z_{j, h, l}\right), \\
\Delta_{h}\left(X^{\mu}\right)\left[\Delta_{h} z_{j, h, l}\right] \equiv & X^{\mu}\left(t_{j, h-1, l}, x_{j, h-1, l}, y_{j, h-1, l}, z_{j, h, l}\right) \\
& -X^{\mu}\left(t_{j, h-1, l}, x_{j, h-1, l}, y_{j, h-1, l}, z_{j, h-1, l}\right) .
\end{aligned}
$$

Lemma 6 In the case of $(j, k, l)$ for $\mu=0,1,2,3$ and $l=1,2, \ldots, j$ and $j=1,2, \ldots, k$ and $k=$ $1,2, \ldots, n$, the following holds:

$$
\begin{aligned}
\left(X^{\mu}\right)_{j, k, l}= & \left(X^{\mu}\right)_{0,0,0}+\sum_{m=1}^{l} \frac{\Delta_{m}\left(X^{\mu}\right)\left[\Delta_{m}\left(x^{\delta}\right)_{0,0, m}\right]}{\Delta_{m}\left(x^{\delta}\right)_{0,0, m}} \Delta_{m}\left(x^{\delta}\right)_{0,0, m} \\
& +\sum_{i=1}^{j} \frac{\Delta_{i}\left(X^{\mu}\right)\left[\Delta_{i}\left(x^{\delta}\right)_{i, 0, l}\right]}{\Delta_{i}\left(x^{\delta}\right)_{i, 0, l}} \Delta_{i}\left(x^{\delta}\right)_{i, 0, l} \\
& +\sum_{h=1}^{k} \frac{\Delta_{h}\left(X^{\mu}\right)\left[\Delta_{h}\left(x^{\delta}\right)_{j, h, l}\right]}{\Delta_{h}\left(x^{\delta}\right)_{j, h, l}} \Delta_{h}\left(x^{\delta}\right)_{j, h, l},
\end{aligned}
$$

where the index is summed over $\delta=0,1,2,3$. 
Proof Using (4.30), (4.36) and (4.42), $\left(X^{\mu}\right)_{j, k, l}$ for $\mu=0,1,2,3$ and $l=1,2, \ldots, j$ and $j=$ $1,2, \ldots, k$ and $k=1,2, \ldots, n$ is disintegrated into

$$
\left(X^{\mu}\right)_{j, k, l}=\left(X^{\mu}\right)_{0,0,0}+\sum_{m=1}^{l} \Delta_{m}\left(X^{\mu}\right)_{0,0, m}+\sum_{i=1}^{j} \Delta_{i}\left(X^{\mu}\right)_{i, 0, l}+\sum_{h=1}^{k} \Delta_{h}\left(X^{\mu}\right)_{j, h, l}
$$

1. Substituting (4.32), (4.33), (4.34) and (4.35) into (4.30) for $\mu=0,1,2,3$ and $m=$ $1,2, \ldots, l$, we obtain

$$
\begin{aligned}
\Delta_{m}\left(X^{\mu}\right)_{0,0, m}= & \Delta_{m}\left(X^{\mu}\right)\left[\Delta_{m} t_{0,0, m}\right]+\Delta_{m}\left(X^{\mu}\right)\left[\Delta_{m} x_{0,0, m}\right] \\
& +\Delta_{m}\left(X^{\mu}\right)\left[\Delta_{m} y_{0,0, m}\right]+\Delta_{m}\left(X^{\mu}\right)\left[\Delta_{m} z_{0,0, m}\right] \\
= & \frac{\Delta_{m}\left(X^{\mu}\right)\left[\Delta_{m}\left(x^{\delta}\right)_{0,0, m}\right]}{\Delta_{m}\left(x^{\delta}\right)_{0,0, m}} \Delta_{m}\left(x^{\delta}\right)_{0,0, m}
\end{aligned}
$$

where the index is summed over $\delta=0,1,2,3$.

2. Substituting (4.38), (4.39), (4.40) and (4.41) into (4.36) for $\mu=0,1,2,3$ and $i, l=$ $1,2, \ldots, j$, we obtain

$$
\begin{aligned}
\Delta_{i}\left(X^{\mu}\right)_{i, 0, l}= & \Delta_{i}\left(X^{\mu}\right)\left[\Delta_{i} t_{i, 0, l}\right]+\Delta_{i}\left(X^{\mu}\right)\left[\Delta_{i} x_{i, 0, l}\right] \\
& +\Delta_{i}\left(X^{\mu}\right)\left[\Delta_{i} y_{i, 0, l}\right]+\Delta_{i}\left(X^{\mu}\right)\left[\Delta_{i} z_{i, 0, l}\right] \\
= & \frac{\Delta_{i}\left(X^{\mu}\right)\left[\Delta_{i}\left(x^{\delta}\right)_{i, 0, l}\right]}{\Delta_{i}\left(x^{\delta}\right)_{i, 0, l}} \Delta_{i}\left(x^{\delta}\right)_{i, 0, l}
\end{aligned}
$$

where the index is summed over $\delta=0,1,2,3$.

3. Substituting (4.44), (4.45), (4.46) and (4.47) into (4.42) for $\mu=0,1,2,3$ and $l=$ $1,2, \ldots, j$ and $h, j=1,2, \ldots, k$, we obtain

$$
\begin{aligned}
\Delta_{h}\left(X^{\mu}\right)_{j, h, l}= & \Delta_{h}\left(X^{\mu}\right)\left[\Delta_{h} t_{j, h, l}\right]+\Delta_{h}\left(X^{\mu}\right)\left[\Delta_{h} x_{j, h, l}\right] \\
& +\Delta_{h}\left(X^{\mu}\right)\left[\Delta_{h} y_{j, h, l}\right]+\Delta_{h}\left(X^{\mu}\right)\left[\Delta_{h} z_{j, h, l}\right] \\
= & \frac{\Delta_{h}\left(X^{\mu}\right)\left[\Delta_{h}\left(x^{\delta}\right)_{j, h, l}\right]}{\Delta_{h}\left(x^{\delta}\right)_{j, h, l}} \Delta_{h}\left(x^{\delta}\right)_{j, h, l}
\end{aligned}
$$

where the index is summed over $\delta=0,1,2,3$.

Substituting (4.50), (4.51) and (4.52) into (4.49), we obtain (4.48).

Q.E.D.

In the similar manner, we obtain the cases of $(j, l, k),(l, j, k),(l, k, j),(k . l, j)$ and $(k, j, l)$ as follows.

Lemma 7 In the case of $(j, l, k)$ for $\mu=0,1,2,3$ and $l=1,2, \ldots, j$ and $j=1,2, \ldots, k$ and $k=$ $1,2, \ldots, n$, the following holds

$$
\begin{aligned}
\left(X^{\mu}\right)_{j, l, k}= & \left(X^{\mu}\right)_{0,0,0}+\sum_{m=1}^{l} \frac{\Delta_{m}\left(X^{\mu}\right)\left[\Delta_{m}\left(x^{\delta}\right)_{0, m, 0}\right]}{\Delta_{m}\left(x^{\delta}\right)_{0, m, 0}} \Delta_{m}\left(x^{\delta}\right)_{0, m, 0} \\
& +\sum_{i=1}^{j} \frac{\Delta_{i}\left(X^{\mu}\right)\left[\Delta_{i}\left(x^{\delta}\right)_{i, l, 0}\right]}{\Delta_{i}\left(x^{\delta}\right)_{i, l, 0}} \Delta_{i}\left(x^{\delta}\right)_{i, l, 0}
\end{aligned}
$$




$$
+\sum_{h=1}^{k} \frac{\Delta_{h}\left(X^{\mu}\right)\left[\Delta_{h}\left(x^{\delta}\right)_{j, l, h}\right]}{\Delta_{h}\left(x^{\delta}\right)_{j, l, h}} \Delta_{h}\left(x^{\delta}\right)_{j, l, h^{\prime}}
$$

where the index is summed over $\delta=0,1,2,3$.

Lemma 8 In the case of $(l, j, k)$ for $\mu=0,1,2,3$ and $l=1,2, \ldots, j$ and $j=1,2, \ldots, k$ and $k=$ $1,2, \ldots, n$, the following holds:

$$
\begin{aligned}
\left(X^{\mu}\right)_{l, j, k}= & \left(X^{\mu}\right)_{0,0,0}+\sum_{m=1}^{l} \frac{\Delta_{m}\left(X^{\mu}\right)\left[\Delta_{m}\left(x^{\delta}\right)_{m, 0,0}\right]}{\Delta_{m}\left(x^{\delta}\right)_{m, 0,0}} \Delta_{m}\left(x^{\delta}\right)_{m, 0,0} \\
& +\sum_{i=1}^{j} \frac{\Delta_{i}\left(X^{\mu}\right)\left[\Delta_{i}\left(x^{\delta}\right)_{l, i, 0}\right]}{\Delta_{i}\left(x^{\delta}\right)_{l, i, 0}} \Delta_{i}\left(x^{\delta}\right)_{l, i, 0} \\
& +\sum_{h=1}^{k} \frac{\Delta_{h}\left(X^{\mu}\right)\left[\Delta_{h}\left(x^{\delta}\right)_{l, j, h}\right]}{\Delta_{h}\left(x^{\delta}\right)_{l, j, h}} \Delta_{h}\left(x^{\delta}\right)_{l, j, h^{\prime}}
\end{aligned}
$$

where the index is summed over $\delta=0,1,2,3$.

Lemma 9 In the case of $(l, k, j)$ for $\mu=0,1,2,3$ and $l=1,2, \ldots, j$ and $j=1,2, \ldots, k$ and $k=$ $1,2, \ldots, n$, the following holds:

$$
\begin{aligned}
\left(X^{\mu}\right)_{l, k, j}= & \left(X^{\mu}\right)_{0,0,0}+\sum_{m=1}^{l} \frac{\Delta_{m}\left(X^{\mu}\right)\left[\Delta_{m}\left(x^{\delta}\right)_{m, 0,0}\right]}{\Delta_{m}\left(x^{\delta}\right)_{m, 0,0}} \Delta_{m}\left(x^{\delta}\right)_{m, 0,0} \\
& +\sum_{i=1}^{j} \frac{\Delta_{i}\left(X^{\mu}\right)\left[\Delta_{i}\left(x^{\delta}\right)_{l, 0, i}\right]}{\Delta_{i}\left(x^{\delta}\right)_{l, 0, i}} \Delta_{i}\left(x^{\delta}\right)_{l, 0, i} \\
& +\sum_{h=1}^{k} \frac{\Delta_{h}\left(X^{\mu}\right)\left[\Delta_{h}\left(x^{\delta}\right)_{l, h, j}\right]}{\Delta_{h}\left(x^{\delta}\right)_{l, h, j}} \Delta_{h}\left(x^{\delta}\right)_{l, h, j}
\end{aligned}
$$

where the index is summed over $\delta=0,1,2,3$.

Lemma 10 In the case of $(k, l, j)$ for $\mu=0,1,2,3$ and $l=1,2, \ldots, j$ and $j=1,2, \ldots, k$ and $k=1,2, \ldots, n$, the following holds:

$$
\begin{aligned}
\left(X^{\mu}\right)_{k, l, j}= & \left(X^{\mu}\right)_{0,0,0}+\sum_{m=1}^{l} \frac{\Delta_{m}\left(X^{\mu}\right)\left[\Delta_{m}\left(x^{\delta}\right)_{0, m, 0}\right]}{\Delta_{m}\left(x^{\delta}\right)_{0, m, 0}} \Delta_{m}\left(x^{\delta}\right)_{0, m, 0} \\
& +\sum_{i=1}^{j} \frac{\Delta_{i}\left(X^{\mu}\right)\left[\Delta_{i}\left(x^{\delta}\right)_{0, l, i}\right]}{\Delta_{i}\left(x^{\delta}\right)_{0, l, i}} \Delta_{i}\left(x^{\delta}\right)_{0, l, i} \\
& +\sum_{h=1}^{k} \frac{\Delta_{h}\left(X^{\mu}\right)\left[\Delta_{h}\left(x^{\delta}\right)_{h, l, j}\right]}{\Delta_{h}\left(x^{\delta}\right)_{h, l, j}} \Delta_{h}\left(x^{\delta}\right)_{h, l, j}
\end{aligned}
$$

where the index is summed over $\delta=0,1,2,3$. 
Lemma 11 In the case of $(k, j, l)$ for $\mu=0,1,2,3$ and $l=1,2, \ldots, j$ and $j=1,2, \ldots, k$ and $k=1,2, \ldots, n$, the following holds:

$$
\begin{aligned}
\left(X^{\mu}\right)_{k, j, l}= & \left(X^{\mu}\right)_{0,0,0}+\sum_{m=1}^{l} \frac{\Delta_{m}\left(X^{\mu}\right)\left[\Delta_{m}\left(x^{\delta}\right)_{0,0, m}\right]}{\Delta_{m}\left(x^{\delta}\right)_{0,0, m}} \Delta_{m}\left(x^{\delta}\right)_{0,0, m} \\
& +\sum_{i=1}^{j} \frac{\Delta_{i}\left(X^{\mu}\right)\left[\Delta_{i}\left(x^{\delta}\right)_{0, i, l}\right]}{\Delta_{i}\left(x^{\delta}\right)_{0, i, l}} \Delta_{i}\left(x^{\delta}\right)_{0, i, l} \\
& +\sum_{h=1}^{k} \frac{\Delta_{h}\left(X^{\mu}\right)\left[\Delta_{h}\left(x^{\delta}\right)_{h, j, l}\right]}{\Delta_{h}\left(x^{\delta}\right)_{h, j, l}} \Delta_{h}\left(x^{\delta}\right)_{h, j, l}
\end{aligned}
$$

where the index is summed over $\delta=0,1,2,3$.

Our triangular triple and quadruple integrals and the divergence theorem of a triangular integral in the $4 \mathrm{D}$ time-space are shown as follows, where $D$ is a domain and $\partial D$ is the boundary of the domain in the 4D time-space.

Definition 7 A triangular hyper-surface triple integral in the 4 D time-space $\iiint_{\partial D} X^{\mu} d^{3} V_{\mu}$ is defined as

$$
\begin{aligned}
& \iiint_{\partial D} X^{\mu} d^{3} V_{\mu} \\
& =\lim _{n \rightarrow \infty} \sum_{k=1}^{n} \sum_{j=1}^{k} \sum_{l=1}^{j}\left[\left(X^{\mu}\right)_{j, k, l}\left(\Delta^{3} V_{\mu}\right)_{j, k, l}+\left(X^{\mu}\right)_{j, l, k}\left(\Delta^{3} V_{\mu}\right)_{j, l, k}+\left(X^{\mu}\right)_{l, j, k}\left(\Delta^{3} V_{\mu}\right)_{l, j, k}\right. \\
& \left.\quad+\left(X^{\mu}\right)_{l, k, j}\left(\Delta^{3} V_{\mu}\right)_{l, k, j}+\left(X^{\mu}\right)_{k, l, j}\left(\Delta^{3} V_{\mu}\right)_{k, l, j}+\left(X^{\mu}\right)_{k, j, l}\left(\Delta^{3} V_{\mu}\right)_{k, j, l}\right]
\end{aligned}
$$

where the index is summed over $\mu=0,1,2,3$.

Definition 8 A triangular quadruple integral, integrands of which are partial differentials in the 4D time-space $\iiint \int_{D} \frac{\partial X^{\prime \prime \prime}}{\partial x^{\prime \prime \prime}} d x^{\prime \prime \prime} d d^{3} V_{\mu}$, is defined as

$$
\begin{aligned}
\iint & \iint_{D} \frac{\partial X^{\prime \prime \prime \mu}}{\partial x^{\prime \prime \prime}} d x^{\prime \prime \prime} d^{3} V_{\mu} \\
= & \lim _{n \rightarrow \infty} \sum_{k=1}^{n} \sum_{j=1}^{k} \sum_{l=1}^{j}\left\{\left[\sum_{m=1}^{l} \frac{\Delta_{m}\left(X^{\mu}\right)\left[\Delta_{m}\left(x^{\delta}\right)_{0,0, m}\right]}{\Delta_{m}\left(x^{\delta}\right)_{0,0, m}} \Delta_{m}\left(x^{\delta}\right)_{0,0, m}\right.\right. \\
& +\sum_{i=1}^{j} \frac{\Delta_{i}\left(X^{\mu}\right)\left[\Delta_{i}\left(x^{\delta}\right)_{i, 0, l}\right]}{\Delta_{i}\left(x^{\delta}\right)_{i, 0, l}} \Delta_{i}\left(x^{\delta}\right)_{i, 0, l} \\
& \left.+\sum_{h=1}^{k} \frac{\Delta_{h}\left(X^{\mu}\right)\left[\Delta_{h}\left(x^{\delta}\right)_{j, h, l}\right]}{\Delta_{h}\left(x^{\delta}\right)_{j, h, l}} \Delta_{h}\left(x^{\delta}\right)_{j, h, l}\right]\left(\Delta^{3} V_{\mu}\right)_{j, k, l} \\
& +\left[\sum_{m=1}^{l} \frac{\Delta_{m}\left(X^{\mu}\right)\left[\Delta_{m}\left(x^{\delta}\right)_{0, m, 0}\right]}{\Delta_{m}\left(x^{\delta}\right)_{0, m, 0}} \Delta_{m}\left(x^{\delta}\right)_{0, m, 0}+\sum_{i=1}^{j} \frac{\Delta_{i}\left(X^{\mu}\right)\left[\Delta_{i}\left(x^{\delta}\right)_{i, l, 0}\right]}{\Delta_{i}\left(x^{\delta}\right)_{i, l, 0}} \Delta_{i}\left(x^{\delta}\right)_{i, l, 0}\right. \\
& \left.+\sum_{h=1}^{k} \frac{\Delta_{h}\left(X^{\mu}\right)\left[\Delta_{h}\left(x^{\delta}\right)_{j, l, h}\right]}{\Delta_{h}\left(x^{\delta}\right)_{j, l, h}} \Delta_{h}\left(x^{\delta}\right)_{j, l, h}\right]\left(\Delta^{3} V_{\mu}\right)_{j, l, k}
\end{aligned}
$$




$$
\begin{aligned}
& +\left[\sum_{m=1}^{l} \frac{\Delta_{m}\left(X^{\mu}\right)\left[\Delta_{m}\left(x^{\delta}\right)_{m, 0,0}\right]}{\Delta_{m}\left(x^{\delta}\right)_{m, 0,0}} \Delta_{m}\left(x^{\delta}\right)_{m, 0,0}+\sum_{i=1}^{j} \frac{\Delta_{i}\left(X^{\mu}\right)\left[\Delta_{i}\left(x^{\delta}\right)_{l, i, 0}\right]}{\Delta_{i}\left(x^{\delta}\right)_{l, i, 0}} \Delta_{i}\left(x^{\delta}\right)_{l, i, 0}\right. \\
& \left.+\sum_{h=1}^{k} \frac{\Delta_{h}\left(X^{\mu}\right)\left[\Delta_{h}\left(x^{\delta}\right)_{l, j, h}\right]}{\Delta_{h}\left(x^{\delta}\right)_{l, j, h}} \Delta_{h}\left(x^{\delta}\right)_{l, j, h}\right]\left(\Delta^{3} V_{\mu}\right)_{l, j, k} \\
& +\left[\sum_{m=1}^{l} \frac{\Delta_{m}\left(X^{\mu}\right)\left[\Delta_{m}\left(x^{\delta}\right)_{m, 0,0}\right]}{\Delta_{m}\left(x^{\delta}\right)_{m, 0,0}} \Delta_{m}\left(x^{\delta}\right)_{m, 0,0}+\sum_{i=1}^{j} \frac{\Delta_{i}\left(X^{\mu}\right)\left[\Delta_{i}\left(x^{\delta}\right)_{l, 0, i}\right]}{\Delta_{i}\left(x^{\delta}\right)_{l, 0, i}} \Delta_{i}\left(x^{\delta}\right)_{l, 0, i}\right. \\
& \left.+\sum_{h=1}^{k} \frac{\Delta_{h}\left(X^{\mu}\right)\left[\Delta_{h}\left(x^{\delta}\right)_{l, h, j}\right]}{\Delta_{h}\left(x^{\delta}\right)_{l, h, j}} \Delta_{h}\left(x^{\delta}\right)_{l, h, j}\right]\left(\Delta^{3} V_{\mu}\right)_{l, k, j} \\
& +\left[\sum_{m=1}^{l} \frac{\Delta_{m}\left(X^{\mu}\right)\left[\Delta_{m}\left(x^{\delta}\right)_{0, m, 0}\right]}{\Delta_{m}\left(x^{\delta}\right)_{0, m, 0}} \Delta_{m}\left(x^{\delta}\right)_{0, m, 0}+\sum_{i=1}^{j} \frac{\Delta_{i}\left(X^{\mu}\right)\left[\Delta_{i}\left(x^{\delta}\right)_{0, l, i}\right]}{\Delta_{i}\left(x^{\delta}\right)_{0, l, i}} \Delta_{i}\left(x^{\delta}\right)_{0, l, i}\right. \\
& \left.+\sum_{h=1}^{k} \frac{\Delta_{h}\left(X^{\mu}\right)\left[\Delta_{h}\left(x^{\delta}\right)_{h, l, j}\right]}{\Delta_{h}\left(x^{\delta}\right)_{h, l, j}} \Delta_{h}\left(x^{\delta}\right)_{h, l, j}\right]\left(\Delta^{3} V_{\mu}\right)_{k, l, j} \\
& +\left[\sum_{m=1}^{l} \frac{\Delta_{m}\left(X^{\mu}\right)\left[\Delta_{m}\left(x^{\delta}\right)_{0,0, m}\right]}{\Delta_{m}\left(x^{\delta}\right)_{0,0, m}} \Delta_{m}\left(x^{\delta}\right)_{0,0, m}+\sum_{i=1}^{j} \frac{\Delta_{i}\left(X^{\mu}\right)\left[\Delta_{i}\left(x^{\delta}\right)_{0, i, l}\right]}{\Delta_{i}\left(x^{\delta}\right)_{0, i, l}} \Delta_{i}\left(x^{\delta}\right)_{0, i, l}\right. \\
& \left.\left.+\sum_{h=1}^{k} \frac{\Delta_{h}\left(X^{\mu}\right)\left[\Delta_{h}\left(x^{\delta}\right)_{h, j, l}\right]}{\Delta_{h}\left(x^{\delta}\right)_{h, j, l}} \Delta_{h}\left(x^{\delta}\right)_{h, j, l}\right]\left(\Delta^{3} V_{\mu}\right)_{k, j, l}\right\}
\end{aligned}
$$

where the indices are summed over $\delta, \mu=0,1,2,3$.

Proposition 5 Denote constants as $C^{0}=C^{t}, C^{1}=C^{x}, C^{2}=C^{y}$ and $C^{3}=C^{z}$, then

$$
\begin{aligned}
C^{\mu} \iiint_{\partial D} d^{3} V_{\mu}= & C^{\mu} \lim _{n \rightarrow \infty} \sum_{k=1}^{n} \sum_{j=1}^{k} \sum_{l=1}^{j}\left[\left(\Delta^{3} V_{\mu}\right)_{j, k, l}+\left(\Delta^{3} V_{\mu}\right)_{j, l, k}+\left(\Delta^{3} V_{\mu}\right)_{l, j, k}\right. \\
& \left.+\left(\Delta^{3} V_{\mu}\right)_{l, k, j}+\left(\Delta^{3} V_{\mu}\right)_{k, l, j}+\left(\Delta^{3} V_{\mu}\right)_{k, j, l}\right]
\end{aligned}
$$

holds, where the index is summed over $\mu=0,1,2,3$.

Proof In the case of $X^{0}=1, X^{1}=0, X^{2}=0$ and $X^{3}=0$, Definition 7 is reduced to be

$$
\begin{aligned}
\iiint_{\partial D} d^{3} V_{t}= & \lim _{n \rightarrow \infty} \sum_{k=1}^{n} \sum_{j=1}^{k} \sum_{l=1}^{j}\left[\left(\Delta^{3} V_{t}\right)_{j, k, l}+\left(\Delta^{3} V_{t}\right)_{j, l, k}+\left(\Delta^{3} V_{t}\right)_{l, j, k}\right. \\
& \left.+\left(\Delta^{3} V_{t}\right)_{l, k, j}+\left(\Delta^{3} V_{t}\right)_{k, l, j}+\left(\Delta^{3} V_{t}\right)_{k, j, l}\right] .
\end{aligned}
$$

In the case of $X^{0}=0, X^{1}=1, X^{2}=0$ and $X^{3}=0$, Definition 7 is reduced to be

$$
\begin{aligned}
\iiint_{\partial D} d^{3} V_{x}= & \lim _{n \rightarrow \infty} \sum_{k=1}^{n} \sum_{j=1}^{k} \sum_{l=1}^{j}\left[\left(\Delta^{3} V_{x}\right)_{j, k, l}+\left(\Delta^{3} V_{x}\right)_{j, l, k}+\left(\Delta^{3} V_{x}\right)_{l, j, k}\right. \\
& \left.+\left(\Delta^{3} V_{x}\right)_{l, k, j}+\left(\Delta^{3} V_{x}\right)_{k, l, j}+\left(\Delta^{3} V_{x}\right)_{k, j, l}\right] .
\end{aligned}
$$


In the case of $X^{0}=0, X^{1}=0, X^{2}=1$ and $X^{3}=0$, Definition 7 is reduced to be

$$
\begin{aligned}
\iiint_{\partial D} d^{3} V_{y}= & \lim _{n \rightarrow \infty} \sum_{k=1}^{n} \sum_{j=1}^{k} \sum_{l=1}^{j}\left[\left(\Delta^{3} V_{y}\right)_{j, k, l}+\left(\Delta^{3} V_{y}\right)_{j, l, k}+\left(\Delta^{3} V_{y}\right)_{l, j, k}\right. \\
& \left.+\left(\Delta^{3} V_{y}\right)_{l, k, j}+\left(\Delta^{3} V_{y}\right)_{k, l, j}+\left(\Delta^{3} V_{y}\right)_{k, j, l}\right] .
\end{aligned}
$$

In the case of $X^{0}=0, X^{1}=0, X^{2}=0$ and $X^{3}=1$, Definition 7 is reduced to be

$$
\begin{aligned}
\iiint_{\partial D} d^{3} V_{z}= & \lim _{n \rightarrow \infty} \sum_{k=1}^{n} \sum_{j=1}^{k} \sum_{l=1}^{j}\left[\left(\Delta^{3} V_{z}\right)_{j, k, l}+\left(\Delta^{3} V_{z}\right)_{j, l, k}+\left(\Delta^{3} V_{z}\right)_{l, j, k}\right. \\
& \left.+\left(\Delta^{3} V_{z}\right)_{l, k, j}+\left(\Delta^{3} V_{z}\right)_{k, l, j}+\left(\Delta^{3} V_{z}\right)_{k, j, l}\right] .
\end{aligned}
$$

A linear combination of (4.61), (4.62), (4.63) and (4.64) is

$$
\begin{aligned}
& C^{t} \iiint_{\partial D} d^{3} V_{t}+C^{x} \iiint_{\partial D} d^{3} V_{x}+C^{y} \iiint_{\partial D} d^{3} V_{y}+C^{z} \iiint_{\partial D} d^{3} V_{z} \\
& =C^{t} \lim _{n \rightarrow \infty} \sum_{k=1}^{n} \sum_{j=1}^{k} \sum_{l=1}^{j}\left[\left(\Delta^{3} V_{t}\right)_{j, k, l}+\left(\Delta^{3} V_{t}\right)_{j, l, k}+\left(\Delta^{3} V_{t}\right)_{l, j, k}\right. \\
& \left.\quad+\left(\Delta^{3} V_{t}\right)_{l, k, j}+\left(\Delta^{3} V_{t}\right)_{k, l, j}+\left(\Delta^{3} V_{t}\right)_{k, j, l}\right] \\
& \quad+C^{x} \lim _{n \rightarrow \infty} \sum_{k=1}^{n} \sum_{j=1}^{k} \sum_{l=1}^{j}\left[\left(\Delta^{3} V_{x}\right)_{j, k, l}+\left(\Delta^{3} V_{x}\right)_{j, l, k}+\left(\Delta^{3} V_{x}\right)_{l, j, k}\right. \\
& \left.\quad+\left(\Delta^{3} V_{x}\right)_{l, k, j}+\left(\Delta^{3} V_{x}\right)_{k, l, j}+\left(\Delta^{3} V_{x}\right)_{k, j, l}\right] \\
& \quad+C^{y} \lim _{n \rightarrow \infty} \sum_{k=1}^{n} \sum_{j=1}^{k} \sum_{l=1}^{j}\left[\left(\Delta^{3} V_{y}\right)_{j, k, l}+\left(\Delta^{3} V_{y}\right)_{j, l, k}+\left(\Delta^{3} V_{y}\right)_{l, j, k}\right. \\
& \left.\quad+\left(\Delta^{3} V_{y}\right)_{l, k, j}+\left(\Delta^{3} V_{y}\right)_{k, l, j}+\left(\Delta^{3} V_{y}\right)_{k, j, l}\right] \\
& \quad+C^{z} \lim _{n \rightarrow \infty} \sum_{k=1}^{n} \sum_{j=1}^{k} \sum_{l=1}^{j}\left[\left(\Delta^{3} V_{z}\right)_{j, k, l}+\left(\Delta^{3} V_{z}\right)_{j, l, k}+\left(\Delta^{3} V_{z}\right)_{l, j, k}\right. \\
& \left.+\left(\Delta^{3} V_{z}\right)_{l, k, j}+\left(\Delta^{3} V_{z}\right)_{k, l, j}+\left(\Delta^{3} V_{z}\right)_{k, j, l}\right] .
\end{aligned}
$$

Theorem 4 shown below is the $4 \mathrm{D}$ version of Theorems 2 and 3 . The condition (4.66) or (4.69) is the $4 \mathrm{D}$ version of the condition (2.17) or (2.39) on the $2 \mathrm{D}$ plane and the condition (3.118) or (3.121) in the 3D space.

Theorem 4 (The divergence theorem of a triangular integral in the 4D time-space) Assume $D$ is a domain and $\partial D$ is the boundary of the domain in the txyz-time-space, expressed in the Cartesian coordinates $(t, x, y, z) \in \mathbb{R}^{4}$. Let $X^{0}=T=T(t, x, y, z), X^{1}=X=X(t, x, y, z)$, $X^{2}=Y=Y(t, x, y, z)$ and $X^{3}=Z=Z(t, x, y, z)$ be partially differentiable functions with respect to $x^{0}=t, x^{1}=x, x^{2}=y$ and $x^{3}=z$ in $D$. In the case of a closed $3 D$ hyper-surface which 
satisfies

$$
C^{\mu} \iiint_{\partial D} d^{3} V_{\mu}=0
$$

the divergence theorem of a triangular integral in the 4D time-space holds:

$$
\oiiint_{\partial D} X^{\mu} d^{3} V_{\mu}=\iiint \int_{D} \frac{\partial X^{\prime \prime \prime} \mu}{\partial x^{\prime \prime \prime} \delta} d x^{\prime \prime \prime} d^{3} V_{\mu}
$$

where the indices are summed over $\delta, \mu=0,1,2,3$ and $C^{\mu}$ are constants.

Proof Substituting (4.48), (4.53), (4.54), (4.55), (4.56) and (4.57) into the right-hand side of (4.29), we obtain

$$
\begin{aligned}
& \sum_{k=1}^{n} \sum_{j=1}^{k} \sum_{l=1}^{j}\left[\left(X^{\mu}\right)_{j, k, l}\left(\Delta^{3} V_{\mu}\right)_{j, k, l}+\left(X^{\mu}\right)_{j, l, k}\left(\Delta^{3} V_{\mu}\right)_{j, l, k}+\left(X^{\mu}\right)_{l, j, k}\left(\Delta^{3} V_{\mu}\right)_{l, j, k}\right. \\
& \left.+\left(X^{\mu}\right)_{l, k, j}\left(\Delta^{3} V_{\mu}\right)_{l, k, j}+\left(X^{\mu}\right)_{k, l, j}\left(\Delta^{3} V_{\mu}\right)_{k, l, j}+\left(X^{\mu}\right)_{k, j, l}\left(\Delta^{3} V_{\mu}\right)_{k, j, l}\right] \\
& =\sum_{k=1}^{n} \sum_{j=1}^{k} \sum_{l=1}^{j}\left\{\left[\sum_{m=1}^{l} \frac{\Delta_{m}\left(X^{\mu}\right)\left[\Delta_{m}\left(x^{\delta}\right)_{0,0, m}\right]}{\Delta_{m}\left(x^{\delta}\right)_{0,0, m}} \Delta_{m}\left(x^{\delta}\right)_{0,0, m}\right.\right. \\
& +\sum_{i=1}^{j} \frac{\Delta_{i}\left(X^{\mu}\right)\left[\Delta_{i}\left(x^{\delta}\right)_{i, 0, l}\right]}{\Delta_{i}\left(x^{\delta}\right)_{i, 0, l}} \Delta_{i}\left(x^{\delta}\right)_{i, 0, l} \\
& \left.+\sum_{h=1}^{k} \frac{\Delta_{h}\left(X^{\mu}\right)\left[\Delta_{h}\left(x^{\delta}\right)_{j, h, l}\right]}{\Delta_{h}\left(x^{\delta}\right)_{j, h, l}} \Delta_{h}\left(x^{\delta}\right)_{j, h, l}\right]\left(\Delta^{3} V_{\mu}\right)_{j, k, l} \\
& +\left[\sum_{m=1}^{l} \frac{\Delta_{m}\left(X^{\mu}\right)\left[\Delta_{m}\left(x^{\delta}\right)_{0, m, 0}\right]}{\Delta_{m}\left(x^{\delta}\right)_{0, m, 0}} \Delta_{m}\left(x^{\delta}\right)_{0, m, 0}+\sum_{i=1}^{j} \frac{\Delta_{i}\left(X^{\mu}\right)\left[\Delta_{i}\left(x^{\delta}\right)_{i, l, 0}\right]}{\Delta_{i}\left(x^{\delta}\right)_{i, l, 0}} \Delta_{i}\left(x^{\delta}\right)_{i, l, 0}\right. \\
& \left.+\sum_{h=1}^{k} \frac{\Delta_{h}\left(X^{\mu}\right)\left[\Delta_{h}\left(x^{\delta}\right)_{j, l, h}\right]}{\Delta_{h}\left(x^{\delta}\right)_{j, l, h}} \Delta_{h}\left(x^{\delta}\right)_{j, l, h}\right]\left(\Delta^{3} V_{\mu}\right)_{j, l, k} \\
& +\left[\sum_{m=1}^{l} \frac{\Delta_{m}\left(X^{\mu}\right)\left[\Delta_{m}\left(x^{\delta}\right)_{m, 0,0}\right]}{\Delta_{m}\left(x^{\delta}\right)_{m, 0,0}} \Delta_{m}\left(x^{\delta}\right)_{m, 0,0}+\sum_{i=1}^{j} \frac{\Delta_{i}\left(X^{\mu}\right)\left[\Delta_{i}\left(x^{\delta}\right)_{l, i, 0}\right]}{\Delta_{i}\left(x^{\delta}\right)_{l, i, 0}} \Delta_{i}\left(x^{\delta}\right)_{l, i, 0}\right. \\
& \left.+\sum_{h=1}^{k} \frac{\Delta_{h}\left(X^{\mu}\right)\left[\Delta_{h}\left(x^{\delta}\right)_{l, j, h}\right]}{\Delta_{h}\left(x^{\delta}\right)_{l, j, h}} \Delta_{h}\left(x^{\delta}\right)_{l, j, h}\right]\left(\Delta^{3} V_{\mu}\right)_{l, j, k} \\
& +\left[\sum_{m=1}^{l} \frac{\Delta_{m}\left(X^{\mu}\right)\left[\Delta_{m}\left(x^{\delta}\right)_{m, 0,0}\right]}{\Delta_{m}\left(x^{\delta}\right)_{m, 0,0}} \Delta_{m}\left(x^{\delta}\right)_{m, 0,0}+\sum_{i=1}^{j} \frac{\Delta_{i}\left(X^{\mu}\right)\left[\Delta_{i}\left(x^{\delta}\right)_{l, 0, i}\right]}{\Delta_{i}\left(x^{\delta}\right)_{l, 0, i}} \Delta_{i}\left(x^{\delta}\right)_{l, 0, i}\right. \\
& \left.+\sum_{h=1}^{k} \frac{\Delta_{h}\left(X^{\mu}\right)\left[\Delta_{h}\left(x^{\delta}\right)_{l, h, j}\right]}{\Delta_{h}\left(x^{\delta}\right)_{l, h, j}} \Delta_{h}\left(x^{\delta}\right)_{l, h, j}\right]\left(\Delta^{3} V_{\mu}\right)_{l, k, j} \\
& +\left[\sum_{m=1}^{l} \frac{\Delta_{m}\left(X^{\mu}\right)\left[\Delta_{m}\left(x^{\delta}\right)_{0, m, 0}\right]}{\Delta_{m}\left(x^{\delta}\right)_{0, m, 0}} \Delta_{m}\left(x^{\delta}\right)_{0, m, 0}+\sum_{i=1}^{j} \frac{\Delta_{i}\left(X^{\mu}\right)\left[\Delta_{i}\left(x^{\delta}\right)_{0, l, i}\right]}{\Delta_{i}\left(x^{\delta}\right)_{0, l, i}} \Delta_{i}\left(x^{\delta}\right)_{0, l, i}\right.
\end{aligned}
$$




$$
\begin{aligned}
& \left.+\sum_{h=1}^{k} \frac{\Delta_{h}\left(X^{\mu}\right)\left[\Delta_{h}\left(x^{\delta}\right)_{h, l, j}\right]}{\Delta_{h}\left(x^{\delta}\right)_{h, l, j}} \Delta_{h}\left(x^{\delta}\right)_{h, l, j}\right]\left(\Delta^{3} V_{\mu}\right)_{k, l, j} \\
& +\left[\sum_{m=1}^{l} \frac{\Delta_{m}\left(X^{\mu}\right)\left[\Delta_{m}\left(x^{\delta}\right)_{0,0, m}\right]}{\Delta_{m}\left(x^{\delta}\right)_{0,0, m}} \Delta_{m}\left(x^{\delta}\right)_{0,0, m}+\sum_{i=1}^{j} \frac{\Delta_{i}\left(X^{\mu}\right)\left[\Delta_{i}\left(x^{\delta}\right)_{0, i, l}\right]}{\Delta_{i}\left(x^{\delta}\right)_{0, i, l}} \Delta_{i}\left(x^{\delta}\right)_{0, i, l}\right. \\
& \left.\left.+\sum_{h=1}^{k} \frac{\Delta_{h}\left(X^{\mu}\right)\left[\Delta_{h}\left(x^{\delta}\right)_{h, j, l}\right]}{\Delta_{h}\left(x^{\delta}\right)_{h, j, l}} \Delta_{h}\left(x^{\delta}\right)_{h, j, l}\right]\left(\Delta^{3} V_{\mu}\right)_{k, j, l}\right\} \\
& +\left(X^{\mu}\right)_{0,0,0} \sum_{k=1}^{n} \sum_{j=1}^{k} \sum_{l=1}^{j}\left[\left(\Delta^{3} V_{\mu}\right)_{j, k, l}+\left(\Delta^{3} V_{\mu}\right)_{j, l, k}+\left(\Delta^{3} V_{\mu}\right)_{l, j, k}\right. \\
& \left.+\left(\Delta^{3} V_{\mu}\right)_{l, k, j}+\left(\Delta^{3} V_{\mu}\right)_{k, l, j}+\left(\Delta^{3} V_{\mu}\right)_{k, j, l}\right]
\end{aligned}
$$

where the indices are summed over $\delta, \mu=0,1,2,3$. Using Proposition 5, (4.66) is rewritten as

$$
\begin{aligned}
& \left(X^{\mu}\right)_{0,0,0} \lim _{n \rightarrow \infty} \sum_{k=1}^{n} \sum_{j=1}^{k} \sum_{l=1}^{j}\left[\left(\Delta^{3} V_{\mu}\right)_{j, k, l}+\left(\Delta^{3} V_{\mu}\right)_{j, l, k}+\left(\Delta^{3} V_{\mu}\right)_{l, j, k}\right. \\
& \left.+\left(\Delta^{3} V_{\mu}\right)_{l, k, j}+\left(\Delta^{3} V_{\mu}\right)_{k, l, j}+\left(\Delta^{3} V_{\mu}\right)_{k, j, l}\right]=0
\end{aligned}
$$

where the index is summed over $\mu=0,1,2,3$. The limit at infinity $n \rightarrow \infty$ of (4.68) under the condition of (4.69) is expressed as (4.67) by Definitions 7 and 8 .

Q.E.D.

Corollary 4 shown below is the 4D version of Corollary 3 . We only consider the case of $\rho=0$ for simplicity.

\section{Corollary 4 In the case of}

$$
\oiiint_{\partial D} X^{\mu} d^{3} V_{\mu}=0
$$

where the index is summed over $\mu=0,1,2,3$, the following holds:

$$
\frac{\partial X^{\mu}}{\partial x^{\mu}}=0
$$

Proof Applying Theorem 4 to the left-hand side of (4.70), it is modified to be

$$
\begin{aligned}
\lim _{n \rightarrow \infty} & \sum_{k=1}^{n} \sum_{j=1}^{k} \sum_{l=1}^{j}\left[\sum_{m=1}^{l} \frac{\Delta_{m}\left(X^{\mu}\right)\left[\Delta_{m}\left(x^{\delta}\right)_{0,0, m}\right]}{\Delta_{m}\left(x^{\delta}\right)_{0,0, m}} \Delta_{m}\left(x^{\delta}\right)_{0,0, m}\right. \\
& \left.+\sum_{i=1}^{j} \frac{\Delta_{i}\left(X^{\mu}\right)\left[\Delta_{i}\left(x^{\delta}\right)_{i, 0, l}\right]}{\Delta_{i}\left(x^{\delta}\right)_{i, 0, l}} \Delta_{i}\left(x^{\delta}\right)_{i, 0, l}+\sum_{h=1}^{k} \frac{\Delta_{h}\left(X^{\mu}\right)\left[\Delta_{h}\left(x^{\delta}\right)_{j, h, l}\right]}{\Delta_{h}\left(x^{\delta}\right)_{j, h, l}} \Delta_{h}\left(x^{\delta}\right)_{j, h, l}\right] \\
& \times \frac{1}{3 !} \varepsilon_{\alpha \beta \gamma \mu}\left\{\Delta_{j}\left(x^{\alpha}\right)_{j, k, l}\left[\Delta_{j}\left(x^{\beta}\right)_{j, k, l}+\Delta_{k}\left(x^{\beta}\right)_{j-1, k, l}\right]\right. \\
& \times\left[\Delta_{j}\left(x^{\gamma}\right)_{j, k, l}+\Delta_{k}\left(x^{\gamma}\right)_{j-1, k, l}+\Delta_{l}\left(x^{\gamma}\right)_{j-1, k-1, l}\right] \\
& +\Delta_{k}\left(x^{\beta}\right)_{j, k, l}\left[\Delta_{k}\left(x^{\gamma}\right)_{j, k, l}+\Delta_{l}\left(x^{\gamma}\right)_{j, k-1, l}\right]\left[\Delta_{k}\left(x^{\alpha}\right)_{j, k, l}+\Delta_{l}\left(x^{\alpha}\right)_{j, k-1, l}+\Delta_{j}\left(x^{\alpha}\right)_{j, k-1, l-1}\right]
\end{aligned}
$$




$$
\begin{aligned}
& \left.+\Delta_{l}\left(x^{\gamma}\right)_{j, k, l}\left[\Delta_{l}\left(x^{\alpha}\right)_{j, k, l}+\Delta_{j}\left(x^{\alpha}\right)_{j, k, l-1}\right]\left[\Delta_{l}\left(x^{\beta}\right)_{j, k, l}+\Delta_{j}\left(x^{\beta}\right)_{j, k, l-1}+\Delta_{k}\left(x^{\beta}\right)_{j-1, k, l-1}\right]\right\} \\
& +\lim _{n \rightarrow \infty} \sum_{k=1}^{n} \sum_{j=1}^{k} \sum_{l=1}^{j}\left[\sum_{m=1}^{l} \frac{\Delta_{m}\left(X^{\mu}\right)\left[\Delta_{m}\left(x^{\delta}\right)_{0, m, 0}\right]}{\Delta_{m}\left(x^{\delta}\right)_{0, m, 0}} \Delta_{m}\left(x^{\delta}\right)_{0, m, 0}\right. \\
& \left.+\sum_{i=1}^{j} \frac{\Delta_{i}\left(X^{\mu}\right)\left[\Delta_{i}\left(x^{\delta}\right)_{i, l, 0}\right]}{\Delta_{i}\left(x^{\delta}\right)_{i, l, 0}} \Delta_{i}\left(x^{\delta}\right)_{i, l, 0}+\sum_{h=1}^{k} \frac{\Delta_{h}\left(X^{\mu}\right)\left[\Delta_{h}\left(x^{\delta}\right)_{j, l, h}\right]}{\Delta_{h}\left(x^{\delta}\right)_{j, l, h}} \Delta_{h}\left(x^{\delta}\right)_{j, l, h}\right] \\
& \times \frac{1}{3 !} \varepsilon_{\alpha \beta \gamma \mu}\left\{\Delta_{j}\left(x^{\alpha}\right)_{j, l, k}\left[\Delta_{j}\left(x^{\beta}\right)_{j, l, k}+\Delta_{l}\left(x^{\beta}\right)_{j-1, l, k}\right]\right. \\
& \times\left[\Delta_{j}\left(x^{\gamma}\right)_{j, l, k}+\Delta_{l}\left(x^{\gamma}\right)_{j-1, l, k}+\Delta_{k}\left(x^{\gamma}\right)_{j-1, l-1, k}\right] \\
& +\Delta_{l}\left(x^{\beta}\right)_{j, l, k}\left[\Delta_{l}\left(x^{\gamma}\right)_{j, l, k}+\Delta_{k}\left(x^{\gamma}\right)_{j, l-1, k}\right]\left[\Delta_{l}\left(x^{\alpha}\right)_{j, l, k}+\Delta_{k}\left(x^{\alpha}\right)_{j, l-1, k}+\Delta_{j}\left(x^{\alpha}\right)_{j, l-1, k-1}\right] \\
& +\Delta_{k}\left(x^{\gamma}\right)_{j, l, k}\left[\Delta_{k}\left(x^{\alpha}\right)_{j, l, k}+\Delta_{j}\left(x^{\alpha}\right)_{j, l, k-1}\right] \\
& \left.\times\left[\Delta_{k}\left(x^{\beta}\right)_{j, l, k}+\Delta_{j}\left(x^{\beta}\right)_{j, l, k-1}+\Delta_{l}\left(x^{\beta}\right)_{j-1, l, k-1}\right]\right\} \\
& +\lim _{n \rightarrow \infty} \sum_{k=1}^{n} \sum_{j=1}^{k} \sum_{l=1}^{j}\left[\sum_{m=1}^{l} \frac{\Delta_{m}\left(X^{\mu}\right)\left[\Delta_{m}\left(x^{\delta}\right)_{m, 0,0}\right]}{\Delta_{m}\left(x^{\delta}\right)_{m, 0,0}} \Delta_{m}\left(x^{\delta}\right)_{m, 0,0}\right. \\
& \left.+\sum_{i=1}^{j} \frac{\Delta_{i}\left(X^{\mu}\right)\left[\Delta_{i}\left(x^{\delta}\right)_{l, i, 0}\right]}{\Delta_{i}\left(x^{\delta}\right)_{l, i, 0}} \Delta_{i}\left(x^{\delta}\right)_{l, i, 0}+\sum_{h=1}^{k} \frac{\Delta_{h}\left(X^{\mu}\right)\left[\Delta_{h}\left(x^{\delta}\right)_{l, j, h}\right]}{\Delta_{h}\left(x^{\delta}\right)_{l, j, h}} \Delta_{h}\left(x^{\delta}\right)_{l, j, h}\right] \\
& \times \frac{1}{3 !} \varepsilon_{\alpha \beta \gamma \mu}\left\{\Delta_{l}\left(x^{\alpha}\right)_{l, j, k}\left[\Delta_{l}\left(x^{\beta}\right)_{l, j, k}+\Delta_{j}\left(x^{\beta}\right)_{l-1, j, k}\right]\right. \\
& \times\left[\Delta_{l}\left(x^{\gamma}\right)_{l, j, k}+\Delta_{j}\left(x^{\gamma}\right)_{l-1, j, k}+\Delta_{k}\left(x^{\gamma}\right)_{l-1, j-1, k}\right] \\
& +\Delta_{j}\left(x^{\beta}\right)_{l, j, k}\left[\Delta_{j}\left(x^{\gamma}\right)_{l, j, k}+\Delta_{k}\left(x^{\gamma}\right)_{l, j-1, k}\right]\left[\Delta_{j}\left(x^{\alpha}\right)_{l, j, k}+\Delta_{k}\left(x^{\alpha}\right)_{l, j-1, k}+\Delta_{l}\left(x^{\alpha}\right)_{l, j-1, k-1}\right] \\
& +\Delta_{k}\left(x^{\gamma}\right)_{l, j, k}\left[\Delta_{k}\left(x^{\alpha}\right)_{l, j, k}+\Delta_{l}\left(x^{\alpha}\right)_{l, j, k-1}\right] \\
& \left.\times\left[\Delta_{k}\left(x^{\beta}\right)_{l, j, k}+\Delta_{l}\left(x^{\beta}\right)_{l, j, k-1}+\Delta_{j}\left(x^{\beta}\right)_{l-1, j, k-1}\right]\right\} \\
& +\lim _{n \rightarrow \infty} \sum_{k=1}^{n} \sum_{j=1}^{k} \sum_{l=1}^{j}\left[\sum_{m=1}^{l} \frac{\Delta_{m}\left(X^{\mu}\right)\left[\Delta_{m}\left(x^{\delta}\right)_{m, 0,0}\right]}{\Delta_{m}\left(x^{\delta}\right)_{m, 0,0}} \Delta_{m}\left(x^{\delta}\right)_{m, 0,0}\right. \\
& \left.+\sum_{i=1}^{j} \frac{\Delta_{i}\left(X^{\mu}\right)\left[\Delta_{i}\left(x^{\delta}\right)_{l, 0, i}\right]}{\Delta_{i}\left(x^{\delta}\right)_{l, 0, i}} \Delta_{i}\left(x^{\delta}\right)_{l, 0, i}+\sum_{h=1}^{k} \frac{\Delta_{h}\left(X^{\mu}\right)\left[\Delta_{h}\left(x^{\delta}\right)_{l, h, j}\right]}{\Delta_{h}\left(x^{\delta}\right)_{l, h, j}} \Delta_{h}\left(x^{\delta}\right)_{l, h, j}\right] \\
& \times \frac{1}{3 !} \varepsilon_{\alpha \beta \gamma \mu}\left\{\Delta_{l}\left(x^{\alpha}\right)_{l, k, j}\left[\Delta_{l}\left(x^{\beta}\right)_{l, k, j}+\Delta_{k}\left(x^{\beta}\right)_{l-1, k, j}\right]\right. \\
& \times\left[\Delta_{l}\left(x^{\gamma}\right)_{l, k, j}+\Delta_{k}\left(x^{\gamma}\right)_{l-1, k, j}+\Delta_{j}\left(x^{\gamma}\right)_{l-1, k-1, j}\right] \\
& +\Delta_{k}\left(x^{\beta}\right)_{l, k, j}\left[\Delta_{k}\left(x^{\gamma}\right)_{l, k, j}+\Delta_{j}\left(x^{\gamma}\right)_{l, k-1, j}\right]\left[\Delta_{k}\left(x^{\alpha}\right)_{l, k, j}+\Delta_{j}\left(x^{\alpha}\right)_{l, k-1, j}+\Delta_{l}\left(x^{\alpha}\right)_{l, k-1, j-1}\right] \\
& \left.+\Delta_{j}\left(x^{\gamma}\right)_{l, k, j}\left[\Delta_{j}\left(x^{\alpha}\right)_{l, k, j}+\Delta_{l}\left(x^{\alpha}\right)_{l, k, j-1}\right]\left[\Delta_{j}\left(x^{\beta}\right)_{l, k, j}+\Delta_{l}\left(x^{\beta}\right)_{l, k, j-1}+\Delta_{k}\left(x^{\beta}\right)_{l-1, k, j-1}\right]\right\} \\
& +\lim _{n \rightarrow \infty} \sum_{k=1}^{n} \sum_{j=1}^{k} \sum_{l=1}^{j}\left[\sum_{m=1}^{l} \frac{\Delta_{m}\left(X^{\mu}\right)\left[\Delta_{m}\left(x^{\delta}\right)_{0, m, 0}\right]}{\Delta_{m}\left(x^{\delta}\right)_{0, m, 0}} \Delta_{m}\left(x^{\delta}\right)_{0, m, 0}\right. \\
& +\sum_{i=1}^{j} \frac{\Delta_{i}\left(X^{\mu}\right)\left[\Delta_{i}\left(x^{\delta}\right)_{0, l, i}\right]}{\Delta_{i}\left(x^{\delta}\right)_{0, l, i}} \Delta_{i}\left(x^{\delta}\right)_{0, l, i}
\end{aligned}
$$




$$
\begin{aligned}
& \left.+\sum_{h=1}^{k} \frac{\Delta_{h}\left(X^{\mu}\right)\left[\Delta_{h}\left(x^{\delta}\right)_{h, l, j}\right]}{\Delta_{h}\left(x^{\delta}\right)_{h, l, j}} \Delta_{h}\left(x^{\delta}\right)_{h, l, j}\right] \\
& \times \frac{1}{3 !} \varepsilon_{\alpha \beta \gamma \mu}\left\{\Delta_{k}\left(x^{\alpha}\right)_{k, l, j}\left[\Delta_{k}\left(x^{\beta}\right)_{k, l, j}+\Delta_{l}\left(x^{\beta}\right)_{k-1, l, j}\right]\right. \\
& \times\left[\Delta_{k}\left(x^{\gamma}\right)_{k, l, j}+\Delta_{l}\left(x^{\gamma}\right)_{k-1, l, j}+\Delta_{j}\left(x^{\gamma}\right)_{k-1, l-1, j}\right] \\
& +\Delta_{l}\left(x^{\beta}\right)_{k, l, j}\left[\Delta_{l}\left(x^{\gamma}\right)_{k, l, j}+\Delta_{j}\left(x^{\gamma}\right)_{k, l-1, j}\right]\left[\Delta_{l}\left(x^{\alpha}\right)_{k, l, j}+\Delta_{j}\left(x^{\alpha}\right)_{k, l-1, j}+\Delta_{k}\left(x^{\alpha}\right)_{k, l-1, j-1}\right] \\
& +\Delta_{j}\left(x^{\gamma}\right)_{k, l, j}\left[\Delta_{j}\left(x^{\alpha}\right)_{k, l, j}+\Delta_{k}\left(x^{\alpha}\right)_{k, l, j-1}\right] \\
& \left.\times\left[\Delta_{j}\left(x^{\beta}\right)_{k, l, j}+\Delta_{k}\left(x^{\beta}\right)_{k, l, j-1}+\Delta_{l}\left(x^{\beta}\right)_{k-1, l, j-1}\right]\right\} \\
& +\lim _{n \rightarrow \infty} \sum_{k=1}^{n} \sum_{j=1}^{k} \sum_{l=1}^{j}\left[\sum_{m=1}^{l} \frac{\Delta_{m}\left(X^{\mu}\right)\left[\Delta_{m}\left(x^{\delta}\right)_{0,0, m}\right]}{\Delta_{m}\left(x^{\delta}\right)_{0,0, m}} \Delta_{m}\left(x^{\delta}\right)_{0,0, m}\right. \\
& +\sum_{i=1}^{j} \frac{\Delta_{i}\left(X^{\mu}\right)\left[\Delta_{i}\left(x^{\delta}\right)_{0, i, l}\right]}{\Delta_{i}\left(x^{\delta}\right)_{0, i, l}} \Delta_{i}\left(x^{\delta}\right)_{0, i, l} \\
& \left.+\sum_{h=1}^{k} \frac{\Delta_{h}\left(X^{\mu}\right)\left[\Delta_{h}\left(x^{\delta}\right)_{h, j, l}\right]}{\Delta_{h}\left(x^{\delta}\right)_{h, j, l}} \Delta_{h}\left(x^{\delta}\right)_{h, j, l}\right] \\
& \times \frac{1}{3 !} \varepsilon_{\alpha \beta \gamma \mu}\left\{\Delta_{k}\left(x^{\alpha}\right)_{k, j, l}\left[\Delta_{k}\left(x^{\beta}\right)_{k, j, l}+\Delta_{j}\left(x^{\beta}\right)_{k-1, j, l}\right]\right. \\
& \times\left[\Delta_{k}\left(x^{\gamma}\right)_{k, j, l}+\Delta_{j}\left(x^{\gamma}\right)_{k-1, j, l}+\Delta_{l}\left(x^{\gamma}\right)_{k-1, j-1, l}\right] \\
& +\Delta_{j}\left(x^{\beta}\right)_{k, j, l}\left[\Delta_{j}\left(x^{\gamma}\right)_{k, j, l}+\Delta_{l}\left(x^{\gamma}\right)_{k, j-1, l}\right]\left[\Delta_{j}\left(x^{\alpha}\right)_{k, j, l}+\Delta_{l}\left(x^{\alpha}\right)_{k, j-1, l}+\Delta_{k}\left(x^{\alpha}\right)_{k, j-1, l-1}\right] \\
& +\Delta_{l}\left(x^{\gamma}\right)_{k, j, l}\left[\Delta_{l}\left(x^{\alpha}\right)_{k, j, l}+\Delta_{k}\left(x^{\alpha}\right)_{k, j, l-1}\right] \\
& \left.\times\left[\Delta_{l}\left(x^{\beta}\right)_{k, j, l}+\Delta_{k}\left(x^{\beta}\right)_{k, j, l-1}+\Delta_{j}\left(x^{\beta}\right)_{k-1, j, l-1}\right]\right\} \\
& =0 \text {, }
\end{aligned}
$$

where the indices are summed over $\alpha, \beta, \gamma, \delta, \mu=0,1,2,3$. In order to hold (4.72) for any value of integral variables, the following fifteen kinds of formulae in three categories are demanded.

1. In the case of $m=1,2, \ldots, l$,

$$
\begin{aligned}
\lim _{\Delta_{m}\left(x^{\delta}\right)_{0,0, m} \rightarrow 0} \frac{\Delta_{m}\left(X^{\mu}\right)\left[\Delta_{m}\left(x^{\delta}\right)_{0,0, m}\right]}{\Delta_{m}\left(x^{\delta}\right)_{0,0, m}} \frac{1}{3 !} \varepsilon_{\alpha \beta \gamma \mu} & =0, \\
\lim _{\Delta_{m}\left(x^{\delta}\right)_{0, m, 0} \rightarrow 0} \frac{\Delta_{m}\left(X^{\mu}\right)\left[\Delta_{m}\left(x^{\delta}\right)_{0, m, 0}\right]}{\Delta_{m}\left(x^{\delta}\right)_{0, m, 0}} \frac{1}{3 !} \varepsilon_{\alpha \beta \gamma \mu} & =0, \\
\lim _{\Delta_{m}\left(x^{\delta}\right)_{m, 0,0} \rightarrow 0} \frac{\Delta_{m}\left(X^{\mu}\right)\left[\Delta_{m}\left(x^{\delta}\right)_{m, 0,0}\right]}{\Delta_{m}\left(x^{\delta}\right)_{m, 0,0}} \frac{1}{3 !} \varepsilon_{\alpha \beta \gamma \mu} & =0
\end{aligned}
$$

for $\alpha, \beta, \gamma, \delta=0,1,2,3$, where the index is summed over $\mu=0,1,2,3$.

2 . In the case of $i, l=1,2, \ldots, j$,

$$
\begin{aligned}
\lim _{\Delta_{i}\left(x^{\delta}\right)_{i, 0, l} \rightarrow 0} \frac{\Delta_{i}\left(X^{\mu}\right)\left[\Delta_{i}\left(x^{\delta}\right)_{i, 0, l}\right]}{\Delta_{i}\left(x^{\delta}\right)_{i, 0, l}} \frac{1}{3 !} \varepsilon_{\alpha \beta \gamma \mu}=0, \\
\lim _{\Delta_{i}\left(x^{\delta}\right)_{i, l, 0} \rightarrow 0} \frac{\Delta_{i}\left(X^{\mu}\right)\left[\Delta_{i}\left(x^{\delta}\right)_{i, l, 0}\right]}{\Delta_{i}\left(x^{\delta}\right)_{i, l, 0}} \frac{1}{3 !} \varepsilon_{\alpha \beta \gamma \mu}=0,
\end{aligned}
$$




$$
\begin{array}{r}
\lim _{\Delta_{i}\left(x^{\delta}\right)_{l, i, 0} \rightarrow 0} \frac{\Delta_{i}\left(X^{\mu}\right)\left[\Delta_{i}\left(x^{\delta}\right)_{l, i, 0}\right]}{\Delta_{i}\left(x^{\delta}\right)_{l, i, 0}} \frac{1}{3 !} \varepsilon_{\alpha \beta \gamma \mu}=0, \\
\lim _{\Delta_{i}\left(x^{\delta}\right)_{l, 0, i} \rightarrow 0} \frac{\Delta_{i}\left(X^{\mu}\right)\left[\Delta_{i}\left(x^{\delta}\right)_{l, 0, i}\right]}{\Delta_{i}\left(x^{\delta}\right)_{l, 0, i}} \frac{1}{3 !} \varepsilon_{\alpha \beta \gamma \mu}=0, \\
\lim _{\Delta_{i}\left(x^{\delta}\right)_{0, l, i} \rightarrow 0} \frac{\Delta_{i}\left(X^{\mu}\right)\left[\Delta_{i}\left(x^{\delta}\right)_{0, l, i}\right]}{\Delta_{i}\left(x^{\delta}\right)_{0, l, i}} \frac{1}{3 !} \varepsilon_{\alpha \beta \gamma \mu}=0, \\
\lim _{\Delta_{i}\left(x^{\delta}\right)_{0, i, l} \rightarrow 0} \frac{\Delta_{i}\left(X^{\mu}\right)\left[\Delta_{i}\left(x^{\delta}\right)_{0, i, l}\right]}{\Delta_{i}\left(x^{\delta}\right)_{0, i, l}} \frac{1}{3 !} \varepsilon_{\alpha \beta \gamma \mu}=0
\end{array}
$$

for $\alpha, \beta, \gamma, \delta=0,1,2,3$, where the index is summed over $\mu=0,1,2,3$.

3. In the case of $l=1,2, \ldots, j$ and $h, j=1,2, \ldots, k$,

$$
\begin{aligned}
\lim _{\Delta_{h}\left(x^{\delta}\right)_{j, h, l} \rightarrow 0} \frac{\Delta_{h}\left(X^{\mu}\right)\left[\Delta_{h}\left(x^{\delta}\right)_{j, h, l}\right]}{\Delta_{h}\left(x^{\delta}\right)_{j, h, l}} \frac{1}{3 !} \varepsilon_{\alpha \beta \gamma \mu} & =0, \\
\lim _{\Delta_{h}\left(x^{\delta}\right)_{j, l, h} \rightarrow 0} \frac{\Delta_{h}\left(X^{\mu}\right)\left[\Delta_{h}\left(x^{\delta}\right)_{j, l, h}\right]}{\Delta_{h}\left(x^{\delta}\right)_{j, l, h}} \frac{1}{3 !} \varepsilon_{\alpha \beta \gamma \mu} & =0, \\
\lim _{\Delta_{h}\left(x^{\delta}\right)_{l, j, h \rightarrow 0}} \frac{\Delta_{h}\left(X^{\mu}\right)\left[\Delta_{h}\left(x^{\delta}\right)_{l, j, h}\right]}{\Delta_{h}\left(x^{\delta}\right)_{l, j, h}} \frac{1}{3 !} \varepsilon_{\alpha \beta \gamma \mu} & =0, \\
\lim _{\Delta_{h}\left(x^{\delta}\right)_{l, h, j} \rightarrow 0} \frac{\Delta_{h}\left(X^{\mu}\right)^{\prime}\left[\Delta_{h}\left(x^{\delta}\right)_{l, h, j}\right]}{\Delta_{h}\left(x^{\delta}\right)_{l, h, j}} \frac{1}{3 !} \varepsilon_{\alpha \beta \gamma \mu} & =0, \\
\lim _{\Delta_{h}\left(x^{\delta}\right)_{h, l, j} \rightarrow 0} \frac{\Delta_{h}\left(X^{\mu}\right)\left[\Delta_{h}\left(x^{\delta}\right)_{h, l, j}\right]}{\Delta_{h}\left(x^{\delta}\right)_{h, l, j}} \frac{1}{3 !} \varepsilon_{\alpha \beta \gamma \mu} & =0, \\
\lim _{\Delta_{h}\left(x^{\delta}\right)_{h, j, l} \rightarrow 0} \frac{\Delta_{h}\left(X^{\mu}\right)\left[\Delta_{h}\left(x^{\delta}\right)_{h, j, l}\right]}{\Delta_{h}\left(x^{\delta}\right)_{h, j, l}} \frac{1}{3 !} \varepsilon_{\alpha \beta \gamma \mu} & =0
\end{aligned}
$$

for $\alpha, \beta, \gamma, \delta=0,1,2,3$, where the index is summed over $\mu=0,1,2,3$.

Multiplying $\varepsilon^{\alpha \beta \gamma \delta}$ to the both sides of the fifteen kinds of formulae (4.73), (4.74), (4.75), (4.76), (4.77), (4.78), (4.79), (4.80), (4.81), (4.82), (4.83), (4.84), (4.85), (4.86) and (4.87), they are reduced to be the differential equation (4.71).

Q.E.D.

\section{Conclusion}

The conventional multiple rectangular integral is merely the inverse operation of the partial differential. It is impossible to regard the conventional multiple rectangular integral as the inverse operation of the total differential in the derivation of the conventional curl or divergence theorem. There should be the inverse operation of the total differential in this sense. Fortunately, both of the curl and the divergence theorems are derived by substituting the total differentials into our new method of a triangular integral. We thus infer that our new method of a triangular integral must be the inverse operation of the total differential.

\section{Appendix A: The concrete forms of increments of the sphere}

1. The upper-right finite area element vector $\Delta^{2} \sigma_{j, k}=\Delta_{j}^{2} \sigma_{j, k}+\Delta_{k}^{2} \sigma_{j, k}$. 
Substituting (3.73) and (3.74) into (3.41), (3.42) and (3.43), we respectively obtain

$$
\begin{aligned}
x_{j, k} & =a \sin \left(\frac{j}{n} \pi\right) \cos \left(2 \frac{k}{n} \pi\right), \\
y_{j, k} & =a \sin \left(\frac{j}{n} \pi\right) \sin \left(2 \frac{k}{n} \pi\right), \\
z_{j, k} & =a \cos \left(\frac{j}{n} \pi\right)
\end{aligned}
$$

for $j=0,1,2, \ldots, k$ and $k=0,1,2, \ldots, n$.

(a) The concrete forms of $\Delta_{j} x_{j, k}, \Delta_{j} y_{j, k}$ and $\Delta_{j} z_{j, k}$ in the case of the sphere.

Applying (A.1), (A.2) and (A.3) to (3.44), (3.45) and (3.46), respectively, we obtain

$$
\begin{aligned}
& \Delta_{j} x_{j, k}=a\left[\sin \left(\frac{j}{n} \pi\right)-\sin \left(\frac{j-1}{n} \pi\right)\right] \cos \left(2 \pi \frac{k}{n}\right), \\
& \Delta_{j} y_{j, k}=a\left[\sin \left(\frac{j}{n} \pi\right)-\sin \left(\frac{j-1}{n} \pi\right)\right] \sin \left(2 \pi \frac{k}{n}\right), \\
& \Delta_{j} z_{j, k}=a\left[\cos \left(\frac{j}{n} \pi\right)-\cos \left(\frac{j-1}{n} \pi\right)\right]
\end{aligned}
$$

for $j=1,2, \ldots, k$ and $k=1,2, \ldots, n$.

(b) The concrete forms of $\Delta_{k} x_{j-1, k}, \Delta_{k} y_{j-1, k}$ and $\Delta_{k} z_{j-1, k}$ in the case of the sphere. Applying (A.1) and (A.2) to (3.47) and (3.48), respectively, we obtain

$$
\begin{aligned}
& \Delta_{k} x_{j-1, k}=a \sin \left(\frac{j-1}{n} \pi\right)\left[\cos \left(2 \pi \frac{k}{n}\right)-\cos \left(2 \pi \frac{k-1}{n}\right)\right], \\
& \Delta_{k} y_{j-1, k}=a \sin \left(\frac{j-1}{n} \pi\right)\left[\sin \left(2 \pi \frac{k}{n}\right)-\sin \left(2 \pi \frac{k-1}{n}\right)\right]
\end{aligned}
$$

for $j=1,2, \ldots, k$ and $k=1,2, \ldots, n$.

(c) The concrete forms of $\Delta_{k} x_{j, k}, \Delta_{k} y_{j, k}$ and $\Delta_{k} z_{j, k}$ in the case of the sphere.

Applying (A.1), (A.2) and (A.3) to (3.50), (3.51) and (3.52), respectively, we obtain

$$
\begin{aligned}
& \Delta_{k} x_{j, k}=a \sin \left(\pi \frac{j}{n}\right)\left[\cos \left(2 \pi \frac{k}{n}\right)-\cos \left(2 \pi \frac{k-1}{n}\right)\right], \\
& \Delta_{k} y_{j, k}=a \sin \left(\pi \frac{j}{n}\right)\left[\sin \left(2 \pi \frac{k}{n}\right)-\sin \left(2 \pi \frac{k-1}{n}\right)\right]
\end{aligned}
$$

for $j=1,2, \ldots, k$ and $k=1,2, \ldots, n$.

(d) The concrete forms of $\Delta_{j} x_{j, k-1}, \Delta_{j} y_{j, k-1}$ and $\Delta_{j} z_{j, k-1}$ in the case of the sphere.

Applying (A.1), (A.2) and (A.3) to (3.53), (3.54) and (3.55), respectively, we obtain

$$
\begin{aligned}
& \Delta_{j} x_{j, k-1}=a\left[\sin \left(\pi \frac{j}{n}\right)-\sin \left(\pi \frac{j-1}{n}\right)\right] \cos \left(2 \pi \frac{k-1}{n}\right), \\
& \Delta_{j} y_{j, k-1}=a\left[\sin \left(\pi \frac{j}{n}\right)-\sin \left(\pi \frac{j-1}{n}\right)\right] \sin \left(2 \pi \frac{k-1}{n}\right),
\end{aligned}
$$




$$
\Delta_{j} z_{j, k-1}=a\left[\cos \left(\pi \frac{j}{n}\right)-\cos \left(\pi \frac{j-1}{n}\right)\right]
$$

for $j=1,2, \ldots, k$ and $k=1,2, \ldots, n$.

2. The lower-left finite area element vector $\Delta^{2} \sigma_{k, j}=\Delta_{k}^{2} \sigma_{k, j}+\Delta_{j}^{2} \sigma_{k, j}$.

Substituting (3.76) and (3.77) into (3.58), (3.59) and (3.60), we respectively obtain

$$
\begin{aligned}
& x_{k, j}=a \sin \left(\frac{k}{n} \pi\right) \cos \left(2 \frac{j}{n} \pi\right), \\
& y_{k, j}=a \sin \left(\frac{k}{n} \pi\right) \sin \left(2 \frac{j}{n} \pi\right), \\
& z_{k, j}=a \cos \left(\frac{k}{n} \pi\right)
\end{aligned}
$$

for $j=0,1,2, \ldots, k$ and $k=0,1,2, \ldots, n$.

(a) The concrete forms of $\Delta_{k} x_{k, j}, \Delta_{k} y_{k, j}$ and $\Delta_{k} z_{k, j}$ in the case of the sphere.

Applying (A.14), (A.15) and (A.16) to (3.61), (3.62) and (3.63), respectively, we obtain

$$
\begin{aligned}
& \Delta_{k} x_{k, j}=a\left[\sin \left(\pi \frac{k}{n}\right)-\sin \left(\pi \frac{k-1}{n}\right)\right] \cos \left(2 \pi \frac{j}{n}\right), \\
& \Delta_{k} y_{k, j}=a\left[\sin \left(\pi \frac{k}{n}\right)-\sin \left(\pi \frac{k-1}{n}\right)\right] \sin \left(2 \pi \frac{j}{n}\right), \\
& \Delta_{k} z_{k, j}=a\left[\cos \left(\pi \frac{k}{n}\right)-\cos \left(\pi \frac{k-1}{n}\right)\right]
\end{aligned}
$$

for $j=1,2, \ldots, k$ and $k=1,2, \ldots, n$.

(b) The concrete forms of $\Delta_{j} x_{k-1, j}, \Delta_{j} y_{k-1, j}$ and $\Delta_{j} z_{k-1, j}$ in the case of the sphere. Applying (A.14) and (A.15) to (3.64) and (3.65), respectively, we obtain

$$
\begin{aligned}
& \Delta_{j} x_{k-1, j}=a \sin \left(\pi \frac{k-1}{n}\right)\left[\cos \left(2 \pi \frac{j}{n}\right)-\cos \left(2 \pi \frac{j-1}{n}\right)\right], \\
& \Delta_{j} y_{k-1, j}=a \sin \left(\pi \frac{k-1}{n}\right)\left[\sin \left(2 \pi \frac{j}{n}\right)-\sin \left(2 \pi \frac{j-1}{n}\right)\right]
\end{aligned}
$$

for $j=1,2, \ldots, k$ and $k=1,2, \ldots, n$.

(c) The concrete forms of $\Delta_{j} x_{k, j}, \Delta_{j} y_{k, j}$ and $\Delta_{j} z_{k, j}$ in the case of the sphere.

Applying (A.14) and (A.15) to (3.67) and (3.68), respectively, we obtain

$$
\begin{aligned}
& \Delta_{j} x_{k, j}=a \sin \left(\pi \frac{k}{n}\right)\left[\cos \left(2 \pi \frac{j}{n}\right)-\cos \left(2 \pi \frac{j-1}{n}\right)\right], \\
& \Delta_{j} y_{k, j}=a \sin \left(\pi \frac{k}{n}\right)\left[\sin \left(2 \pi \frac{j}{n}\right)-\sin \left(2 \pi \frac{j-1}{n}\right)\right]
\end{aligned}
$$

for $j=1,2, \ldots, k$ and $k=1,2, \ldots, n$.

(d) The concrete forms of $\Delta_{k} x_{k, j-1}, \Delta_{k} y_{k, j-1}$ and $\Delta_{k} z_{k, j-1}$ in the case of the sphere. Applying (A.14), (A.15) and (A.16) to (3.70), (3.71) and (3.72), respectively, we obtain

$$
\Delta_{k} x_{k, j-1}=a\left[\sin \left(\pi \frac{k}{n}\right)-\sin \left(\pi \frac{k-1}{n}\right)\right] \cos \left(2 \pi \frac{j-1}{n}\right)
$$




$$
\begin{aligned}
& \Delta_{k} y_{k, j-1}=a\left[\sin \left(\pi \frac{k}{n}\right)-\sin \left(\pi \frac{k-1}{n}\right)\right] \sin \left(2 \pi \frac{j-1}{n}\right), \\
& \Delta_{k} z_{k, j-1}=a\left[\cos \left(\pi \frac{k}{n}\right)-\cos \left(\pi \frac{k-1}{n}\right)\right]
\end{aligned}
$$

for $j=1,2, \ldots, k$ and $k=1,2, \ldots, n$.

\section{Appendix B: The surface area and the volume of the sphere}

1. The upper-right finite area element vector $\overrightarrow{\Delta^{2} \sigma_{j, k}}=\overrightarrow{\Delta_{j}^{2} \sigma_{j, k}}+\overrightarrow{\Delta_{k}^{2} \sigma_{j, k}}$.

(a) In the case of a double increment $\overrightarrow{\Delta_{j}^{2} \sigma_{j, k}}$.

Substituting (A.8), (3.49) and (A.6) into (3.10), the value of $\lim _{n \rightarrow \infty} \sum_{k=1}^{n} \sum_{j=1}^{k} b\left(\overrightarrow{n_{j, k}}\right)_{x} \times$ $\left(\overrightarrow{\Delta_{j}^{2} \sigma_{j, k}}\right)_{x}$ is

$$
\begin{aligned}
\lim _{n \rightarrow \infty} & \sum_{k=1}^{n} \sum_{j=1}^{k} b\left(\overrightarrow{n_{j, k}}\right)_{x}\left(\overrightarrow{\Delta_{j}^{2} \sigma_{j, k}}\right)_{x} \\
= & \frac{a^{2} b}{2} \lim _{n \rightarrow \infty} \sum_{k=1}^{n} \sum_{j=1}^{k} \sin \left(\pi \frac{j}{n}\right) \cos \left(2 \pi \frac{k}{n}\right) \\
\times & \left\{-\sin \left(\frac{j-1}{n} \pi\right)\left[\sin \left(2 \pi \frac{k}{n}\right)-\sin \left(2 \pi \frac{k-1}{n}\right)\right]\right. \\
\times & {\left.\left[\cos \left(\frac{j}{n} \pi\right)-\cos \left(\frac{j-1}{n} \pi\right)\right]\right\} } \\
= & \frac{1}{3} \pi a^{2} b .
\end{aligned}
$$

Substituting (A.7), (3.49) and (A.6) into (3.11), the value of $\lim _{n \rightarrow \infty} \sum_{k=1}^{n} \sum_{j=1}^{k} b\left(\overrightarrow{n_{j, k}}\right)_{y} \times$ $\left(\overrightarrow{\Delta_{j}^{2} \sigma_{j, k}}\right)_{y}$ is

$$
\begin{aligned}
\lim _{n \rightarrow \infty} & \sum_{k=1}^{n} \sum_{j=1}^{k} b\left(\overrightarrow{n_{j, k}}\right)_{y}\left(\overrightarrow{\Delta_{j}^{2} \sigma_{j, k}}\right)_{y} \\
= & \frac{a^{2} b}{2} \lim _{n \rightarrow \infty} \sum_{k=1}^{n} \sum_{j=1}^{k} \sin \left(\pi \frac{j}{n}\right) \sin \left(2 \pi \frac{k}{n}\right) \\
& \times\left\{\left[\cos \left(\frac{j}{n} \pi\right)-\cos \left(\frac{j-1}{n} \pi\right)\right] \sin \left(\frac{j-1}{n} \pi\right)\right. \\
& \left.\times\left[\cos \left(2 \pi \frac{k}{n}\right)-\cos \left(2 \pi \frac{k-1}{n}\right)\right]\right\} \\
= & \frac{1}{3} \pi a^{2} b .
\end{aligned}
$$

Substituting (A.7), (A.8), (A.4) and (A.5) into (3.12), the value of

$$
\lim _{n \rightarrow \infty} \sum_{k=1}^{n} \sum_{j=1}^{k} b\left(\overrightarrow{n_{j, k}}\right)_{z}\left(\overrightarrow{\Delta_{j}^{2} \sigma_{j, k}}\right)_{z}
$$


is

$$
\begin{aligned}
\lim _{n \rightarrow \infty} & \sum_{k=1}^{n} \sum_{j=1}^{k} b\left(\overrightarrow{n_{j, k}}\right)_{z}\left(\overrightarrow{\Delta_{j}^{2} \sigma_{j, k}}\right)_{z} \\
= & \frac{a^{2} b}{2} \lim _{n \rightarrow \infty} \sum_{k=1}^{n} \sum_{j=1}^{k} \cos \left(\pi \frac{j}{n}\right) \\
& \times\left\{\cos \left(2 \pi \frac{k}{n}\right)\left[\sin \left(\frac{j}{n} \pi\right)-\sin \left(\frac{j-1}{n} \pi\right)\right]\right. \\
& \times \sin \left(\frac{j-1}{n} \pi\right)\left[\sin \left(2 \pi \frac{k}{n}\right)-\sin \left(2 \pi \frac{k-1}{n}\right)\right] \\
& -\sin \left(\frac{j-1}{n} \pi\right)\left[\cos \left(2 \pi \frac{k}{n}\right)-\cos \left(2 \pi \frac{k-1}{n}\right)\right] \\
& \left.\times \sin \left(2 \pi \frac{k}{n}\right)\left[\sin \left(\frac{j}{n} \pi\right)-\sin \left(\frac{j-1}{n} \pi\right)\right]\right\} \\
= & \frac{1}{3} \pi a^{2} b .
\end{aligned}
$$

(b) In the case of a double increment $\overrightarrow{\Delta_{k}^{2} \sigma_{j, k}}$.

Substituting (A.12), (A.13), (A.10) and (3.52) into (3.16), the value of

$$
\lim _{n \rightarrow \infty} \sum_{k=1}^{n} \sum_{j=1}^{k} b\left(\overrightarrow{n_{j, k}}\right)_{x}\left(\overrightarrow{\Delta_{k}^{2} \sigma_{j, k}}\right)_{x}
$$

is

$$
\begin{aligned}
& \lim _{n \rightarrow \infty} \sum_{k=1}^{n} \sum_{j=1}^{k} b\left(\overrightarrow{n_{j, k}}\right)_{x}\left(\overrightarrow{\Delta_{k}^{2} \sigma_{j, k}}\right)_{x} \\
&= \frac{a^{2} b}{2} \lim _{n \rightarrow \infty} \sum_{k=1}^{n} \sum_{j=1}^{k} \sin \left(\pi \frac{j}{n}\right) \cos \left(2 \pi \frac{k}{n}\right) \\
& \quad \times \sin \left(\pi \frac{j}{n}\right)\left[\sin \left(2 \pi \frac{k}{n}\right)-\sin \left(2 \pi \frac{k-1}{n}\right)\right]\left[\cos \left(\pi \frac{j}{n}\right)-\cos \left(\pi \frac{j-1}{n}\right)\right] \\
&=\frac{1}{3} \pi a^{2} b .
\end{aligned}
$$

Substituting (A.11), (A.13), (A.9) and (3.52) into (3.17), the value of

$$
\lim _{n \rightarrow \infty} \sum_{k=1}^{n} \sum_{j=1}^{k} b\left(\overrightarrow{n_{j, k}}\right)_{y}\left(\overrightarrow{\Delta_{k}^{2} \sigma_{j, k}}\right)_{y}
$$

is

$$
\lim _{n \rightarrow \infty} \sum_{k=1}^{n} \sum_{j=1}^{k} b\left(\overrightarrow{n_{j, k}}\right)_{y}\left(\overrightarrow{\Delta_{k}^{2} \sigma_{j, k}}\right)_{y}
$$




$$
\begin{aligned}
= & \frac{-a^{2} b}{2} \lim _{n \rightarrow \infty} \sum_{k=1}^{n} \sum_{j=1}^{k} \sin \left(\pi \frac{j}{n}\right) \sin \left(2 \pi \frac{k}{n}\right) \\
& \times\left[\cos \left(\pi \frac{j}{n}\right)-\cos \left(\pi \frac{j-1}{n}\right)\right] \sin \left(\pi \frac{j}{n}\right)\left[\cos \left(2 \pi \frac{k}{n}\right)-\cos \left(2 \pi \frac{k-1}{n}\right)\right] \\
= & \frac{1}{3} \pi a^{2} b .
\end{aligned}
$$

Substituting (A.11), (A.12), (A.9) and (A.10) into (3.18), the value of

$$
\lim _{n \rightarrow \infty} \sum_{k=1}^{n} \sum_{j=1}^{k} b\left(\overrightarrow{n_{j, k}}\right)_{z}\left(\overrightarrow{\Delta_{k}^{2} \sigma_{j, k}}\right)_{z}
$$

is

$$
\begin{aligned}
& \lim _{n \rightarrow \infty} \sum_{k=1}^{n} \sum_{j=1}^{k} b\left(\overrightarrow{n_{j, k}}\right)_{z}\left(\overrightarrow{\Delta_{k}^{2} \sigma_{j, k}}\right)_{z} \\
&= \frac{a^{2} b}{2} \lim _{n \rightarrow \infty} \sum_{k=1}^{n} \sum_{j=1}^{k} \cos \left(\pi \frac{j}{n}\right) \\
& \quad \times\left\{\sin \left(\pi \frac{j}{n}\right)\left[\cos \left(2 \pi \frac{k}{n}\right)-\cos \left(2 \pi \frac{k-1}{n}\right)\right]\right. \\
& \quad \times \sin \left(2 \pi \frac{k-1}{n}\right)\left[\sin \left(\pi \frac{j}{n}\right)-\sin \left(\pi \frac{j-1}{n}\right)\right] \\
& \quad-\cos \left(2 \pi \frac{k-1}{n}\right)\left[\sin \left(\pi \frac{j}{n}\right)-\sin \left(\pi \frac{j-1}{n}\right)\right] \\
&\left.\quad \times \sin \left(\pi \frac{j}{n}\right)\left[\sin \left(2 \pi \frac{k}{n}\right)-\sin \left(2 \pi \frac{k-1}{n}\right)\right]\right\} \\
&=\frac{1}{3} \pi a^{2} b .
\end{aligned}
$$

2. The lower-left finite area element vector $\overrightarrow{\Delta^{2} \sigma_{k, j}}=\overrightarrow{\Delta_{k}^{2} \sigma_{k, j}}+\overrightarrow{\Delta_{j}^{2} \sigma_{k, j}}$.

(a) In the case of a double increment $\overrightarrow{\Delta_{k}^{2} \sigma_{k, j}}$.

Substituting (A.21), (3.66), (A.18) and (A.19) into (3.21), the value of

$$
\lim _{n \rightarrow \infty} \sum_{k=1}^{n} \sum_{j=1}^{k} b\left(\overrightarrow{n_{k, j}}\right)_{x}\left(\overrightarrow{\Delta_{k}^{2} \sigma_{k, j}}\right)_{x}
$$

is

$$
\begin{aligned}
\lim _{n \rightarrow \infty} \sum_{k=1}^{n} \sum_{j=1}^{k} b\left(\overrightarrow{n_{k, j}}\right)_{x}\left(\overrightarrow{\Delta_{k}^{2} \sigma_{k, j}}\right)_{x} \\
=\frac{a^{2} b}{2} \lim _{n \rightarrow \infty} \sum_{k=1}^{n} \sum_{j=1}^{k} \sin \left(\pi \frac{k}{n}\right) \cos \left(2 \pi \frac{j}{n}\right) \\
\quad \times\left\{-\sin \left(\frac{k-1}{n} \pi\right)\left[\sin \left(2 \pi \frac{j}{n}\right)-\sin \left(2 \pi \frac{j-1}{n}\right)\right]\left[\cos \left(\frac{k}{n} \pi\right)-\cos \left(\frac{k-1}{n} \pi\right)\right]\right\}
\end{aligned}
$$




$$
=\frac{1}{3} \pi a^{2} b .
$$

Substituting (A.20), (3.66), (A.17) and (A.19) into (3.22), the value of

$$
\lim _{n \rightarrow \infty} \sum_{k=1}^{n} \sum_{j=1}^{k} b\left(\overrightarrow{n_{k, j}}\right)_{y}\left(\overrightarrow{\Delta_{k}^{2} \sigma_{k, j}}\right)_{y}
$$

is

$$
\begin{aligned}
\lim _{n \rightarrow \infty} & \sum_{k=1}^{n} \sum_{j=1}^{k} b\left(\overrightarrow{n_{k, j}}\right)_{y}\left(\overrightarrow{\Delta_{k}^{2} \sigma_{k, j}}\right)_{y} \\
= & \frac{a^{2} b}{2} \lim _{n \rightarrow \infty} \sum_{k=1}^{n} \sum_{j=1}^{k} \sin \left(\pi \frac{k}{n}\right) \sin \left(2 \pi \frac{j}{n}\right) \\
& \times\left\{\left[\cos \left(\frac{k}{n} \pi\right)-\cos \left(\frac{k-1}{n} \pi\right)\right] \sin \left(\frac{k-1}{n} \pi\right)\left[\cos \left(2 \pi \frac{j}{n}\right)-\cos \left(2 \pi \frac{j-1}{n}\right)\right]\right\} \\
= & \frac{1}{3} \pi a^{2} b .
\end{aligned}
$$

Substituting (A.20), (A.21), (A.17) and (A.18) into (3.23), the value of

$$
\lim _{n \rightarrow \infty} \sum_{k=1}^{n} \sum_{j=1}^{k} b\left(\overrightarrow{n_{k, j}}\right)_{z}\left(\overrightarrow{\Delta_{k}^{2} \sigma_{k, j}}\right)_{z}
$$

is

$$
\begin{aligned}
\lim _{n \rightarrow \infty} & \sum_{k=1}^{n} \sum_{j=1}^{k} b\left(\overrightarrow{n_{k, j}}\right)_{z}\left(\overrightarrow{\Delta_{k}^{2} \sigma_{k, j}}\right)_{z} \\
= & \frac{a^{2} b}{2} \lim _{n \rightarrow \infty} \sum_{k=1}^{n} \sum_{j=1}^{k} \cos \left(\pi \frac{k}{n}\right) \\
& \times\left\{\cos \left(2 \pi \frac{j}{n}\right)\left[\sin \left(\frac{k}{n} \pi\right)-\sin \left(\frac{k-1}{n} \pi\right)\right]\right. \\
& \times \sin \left(\frac{k-1}{n} \pi\right)\left[\sin \left(2 \pi \frac{j}{n}\right)-\sin \left(2 \pi \frac{j-1}{n}\right)\right] \\
& -\sin \left(\frac{k-1}{n} \pi\right)\left[\cos \left(2 \pi \frac{j}{n}\right)-\cos \left(2 \pi \frac{j-1}{n}\right)\right] \\
& \left.\times \sin \left(2 \pi \frac{j}{n}\right)\left[\sin \left(\frac{k}{n} \pi\right)-\sin \left(\frac{k-1}{n} \pi\right)\right]\right\} \\
= & \frac{1}{3} \pi a^{2} b .
\end{aligned}
$$

(b) In the case of a double increment $\overrightarrow{\Delta_{j}^{2} \sigma_{k, j}}$. 
Substituting (A.25), (A.26), (A.23) and (3.69) into (3.26), the value of

$$
\lim _{n \rightarrow \infty} \sum_{k=1}^{n} \sum_{j=1}^{k} b\left(\overrightarrow{n_{k, j}}\right)_{x}\left(\overrightarrow{\Delta_{j}^{2} \sigma_{k, j}}\right)_{x}
$$

is

$$
\begin{aligned}
\lim _{n \rightarrow \infty} & \sum_{k=1}^{n} \sum_{j=1}^{k} b\left(\overrightarrow{n_{k, j}}\right)_{x}\left(\overrightarrow{\Delta_{j}^{2} \sigma_{k, j}}\right)_{x} \\
= & \frac{-a^{2} b}{2} \lim _{n \rightarrow \infty} \sum_{k=1}^{n} \sum_{j=1}^{k} \sin \left(\pi \frac{k}{n}\right) \cos \left(2 \pi \frac{j}{n}\right) \\
& \times \sin \left(\pi \frac{k}{n}\right)\left[\sin \left(2 \pi \frac{j}{n}\right)-\sin \left(2 \pi \frac{j-1}{n}\right)\right]\left[\cos \left(\pi \frac{k}{n}\right)-\cos \left(\pi \frac{k-1}{n}\right)\right] \\
= & \frac{1}{3} \pi a^{2} b .
\end{aligned}
$$

Substituting (A.24), (A.26), (A.22) and (3.69) into (3.27), the value of

$$
\lim _{n \rightarrow \infty} \sum_{k=1}^{n} \sum_{j=1}^{k} b\left(\overrightarrow{n_{k, j}}\right)_{y}\left(\overrightarrow{\Delta_{j}^{2} \sigma_{k, j}}\right)_{y}
$$

is

$$
\begin{aligned}
\lim _{n \rightarrow \infty} & \sum_{k=1}^{n} \sum_{j=1}^{k} b\left(\overrightarrow{n_{k, j}}\right)_{y}\left(\overrightarrow{\Delta_{j}^{2} \sigma_{k, j}}\right)_{y} \\
= & \frac{a^{2} b}{2} \lim _{n \rightarrow \infty} \sum_{k=1}^{n} \sum_{j=1}^{k} \sin \left(\pi \frac{k}{n}\right) \sin \left(2 \pi \frac{j}{n}\right) \\
& \times\left[\cos \left(\pi \frac{k}{n}\right)-\cos \left(\pi \frac{k-1}{n}\right)\right] \sin \left(\pi \frac{k}{n}\right)\left[\cos \left(2 \pi \frac{j}{n}\right)-\cos \left(2 \pi \frac{j-1}{n}\right)\right] \\
= & \frac{1}{3} \pi a^{2} b .
\end{aligned}
$$

Substituting (A.24), (A.25), (A.22) and (A.23) into (3.28), the value of

$$
\lim _{n \rightarrow \infty} \sum_{k=1}^{n} \sum_{j=1}^{k} b\left(\overrightarrow{n_{k, j}}\right)_{z}\left(\overrightarrow{\Delta_{j}^{2} \sigma_{k, j}}\right)_{z}
$$

is

$$
\begin{aligned}
\lim _{n \rightarrow \infty} & \sum_{k=1}^{n} \sum_{j=1}^{k} b\left(\overrightarrow{n_{k, j}}\right)_{z}\left(\overrightarrow{\Delta_{j}^{2} \sigma_{k, j}}\right)_{z} \\
= & \frac{-a^{2} b}{2} \lim _{n \rightarrow \infty} \sum_{k=1}^{n} \sum_{j=1}^{k} \cos \left(\pi \frac{k}{n}\right) \\
& \times\left\{\sin \left(\pi \frac{k}{n}\right)\left[\cos \left(2 \pi \frac{j}{n}\right)-\cos \left(2 \pi \frac{j-1}{n}\right)\right]\right.
\end{aligned}
$$




$$
\begin{aligned}
& \times \sin \left(2 \pi \frac{j-1}{n}\right)\left[\sin \left(\pi \frac{k}{n}\right)-\sin \left(\pi \frac{k-1}{n}\right)\right] \\
& -\cos \left(2 \pi \frac{j-1}{n}\right)\left[\sin \left(\pi \frac{k}{n}\right)-\sin \left(\pi \frac{k-1}{n}\right)\right] \\
& \left.\times \sin \left(\pi \frac{k}{n}\right)\left[\sin \left(2 \pi \frac{j}{n}\right)-\sin \left(2 \pi \frac{j-1}{n}\right)\right]\right\} \\
& =\frac{1}{3} \pi a^{2} b .
\end{aligned}
$$

\section{Appendix C: The condition of the closed surface of the sphere}

In the case of the sphere, the calculations of (3.130) in detail are shown as follows:

$$
\begin{aligned}
& \lim _{n \rightarrow \infty} \sum_{k=1}^{n} \sum_{j=1}^{k}\left(\overrightarrow{\Delta^{2} \sigma_{j, k}}\right)_{z}=\lim _{n \rightarrow \infty} \sum_{k=1}^{n} \sum_{j=1}^{k}\left[\left(\overrightarrow{\Delta_{j}^{2} \sigma_{j, k}}\right)_{z}+\left(\overrightarrow{\Delta_{k}^{2} \sigma_{j, k}}\right)_{z}\right] \\
& =\frac{a^{2}}{2} \lim _{n \rightarrow \infty} \sum_{k=1}^{n} \sum_{j=1}^{k}\left[\sin \left(\frac{j}{n} \pi\right)-\sin \left(\frac{j-1}{n} \pi\right)\right] \\
& \times\left\{\left[\sin \left(\frac{j-1}{n} \pi\right) \cos \left(2 \frac{k}{n} \pi\right)+\sin \left(\frac{j}{n} \pi\right) \cos \left(2 \frac{k-1}{n} \pi\right)\right]\right. \\
& \times\left[\sin \left(2 \frac{k}{n} \pi\right)-\sin \left(2 \frac{k-1}{n} \pi\right)\right] \\
& -\left[\sin \left(\frac{j-1}{n} \pi\right) \sin \left(2 \frac{k}{n} \pi\right)+\sin \left(\frac{j}{n} \pi\right) \sin \left(2 \frac{k-1}{n} \pi\right)\right] \\
& \left.\times\left[\cos \left(2 \frac{k}{n} \pi\right)-\cos \left(2 \frac{k-1}{n} \pi\right)\right]\right\} \\
& =\frac{1}{2} \pi a^{2} \text {, } \\
& \lim _{n \rightarrow \infty} \sum_{k=1}^{n} \sum_{j=1}^{k}\left(\overrightarrow{\Delta^{2} \sigma_{k, j}}\right)_{z}=\lim _{n \rightarrow \infty} \sum_{k=1}^{n} \sum_{j=1}^{k}\left[\left(\overrightarrow{\Delta_{k}^{2} \sigma_{k, j}}\right)_{z}+\left(\overrightarrow{\Delta_{j}^{2} \sigma_{k, j}}\right)_{z}\right] \\
& =\frac{a^{2}}{2} \lim _{n \rightarrow \infty} \sum_{k=1}^{n} \sum_{j=1}^{k}\left[\sin \left(\frac{k}{n} \pi\right)-\sin \left(\frac{k-1}{n} \pi\right)\right] \\
& \times\left\{\left[\sin \left(\frac{k-1}{n} \pi\right) \cos \left(2 \frac{j}{n} \pi\right)+\sin \left(\frac{k}{n} \pi\right) \cos \left(2 \frac{j-1}{n} \pi\right)\right]\right. \\
& \times\left[\sin \left(2 \frac{j}{n} \pi\right)-\sin \left(2 \frac{j-1}{n} \pi\right)\right] \\
& -\left[\sin \left(\frac{k-1}{n} \pi\right) \sin \left(2 \frac{j}{n} \pi\right)+\sin \left(\frac{k}{n} \pi\right) \sin \left(2 \frac{j-1}{n} \pi\right)\right] \\
& \left.\times\left[\cos \left(2 \frac{j}{n} \pi\right)-\cos \left(2 \frac{j-1}{n} \pi\right)\right]\right\} \\
& =-\frac{1}{2} \pi a^{2} \text {. }
\end{aligned}
$$


Competing interests

The author declares that he has no competing interests.

\section{Acknowledgements}

The author thanks Professor Susumu Ishikawa at Fukuoka Institute of Technology for refining this theory.

Received: 14 July 2012 Accepted: 10 September 2012 Published: 25 September 2012

\section{References}

1. Lebesgue, H: Intégrale, longueur, aire. Ann. Mat. Pura Appl. 7(3), 231-359 (1902) (Thèses présentées à la Faculté des sciences de Paris pour obtenir le grade de Docteur ès sciences mathématiques. Bernardoni de C. Rebeschini, Milano (1902))

2. Lebesgue, H: Leçon sur L'intégration et la Recherche des Fonctions Primitives. Gauthier-Villars, Paris (1904)

3. Cartan, É: Sur l'intégration des systèmes d'équations aux différentielles totales. Ann. Sci. Éc. Norm. Super. 18(3), 241-311 (1901)

4. Tokunaga, K: The curl theorem of a triangular integral. Adv. Differ. Equ. 2012, 23 (2012). doi:10.1186/1687-1847-2012-23

5. Bramble, JH, Zlámal, M: Triangular elements in the finite element method. Math. Comput. 24(112), 809-820 (1970)

doi:10.1186/1687-1847-2012-168

Cite this article as: Tokunaga: The divergence theorem of a triangular integral. Advances in Difference Equations 2012 2012:168.

\section{Submit your manuscript to a SpringerOpen ${ }^{\circ}$ journal and benefit from:}

- Convenient online submission

- Rigorous peer review

- Immediate publication on acceptance

- Open access: articles freely available online

- High visibility within the field

- Retaining the copyright to your article 